\title{
Moduli spaces of bundles over two-dimensional orders
}

\author{
Dissertation \\ zur Erlangung des mathematisch-naturwissenschaftlichen Doktorgrades \\ "Doctor rerum naturalium" \\ der Georg-August-Universität Göttingen
}

im Promotionsprogramm der PhD School of Mathematical Science (SMS)

der Georg-August University School of Science (GAUSS)

vorgelegt von

Fabian Reede

aus

Höxter

Göttingen, 2013 


\section{Betreuungsausschuss}

Prof. Dr. Ulrich Stuhler, Mathematisches Institut

Prof. Dr. Viktor Pidstrygach, Mathematisches Institut

\section{Mitglieder der Prüfungskommision}

Referent: Prof. Dr. Ulrich Stuhler, Mathematisches Institut

Korreferent: Prof. Dr. Viktor Pidstrygach, Mathematisches Institut

Weitere Mitglieder der Prüfungskommision:

Prof. Dr. Thomas Schick, Mathematisches Institut

Prof. Dr. Anja Sturm, Institut für Mathematische Stochastik

Prof. Dr. Thorsten Hohage, Institut für Numerische und Angewandte Mathematik

Prof. Dr. Max Wardetzky, Institut für Numerische und Angewandte Mathematik

Tag der mündlichen Prüfung: 23.04.2013 


\section{Introduction}

Vector bundles are ubiquitous in many areas of mathematics and theoretical physics. In algebraic topology or differential geometry many interesting vector bundles are vector bundles associated to a manifold $M$. Well known examples are the tangent bundle $T M$, the bundle of $k$-forms $\Lambda^{k}(M)$ or the bundle of $(r, s)$-tensors $T^{(r, s)} M$. With the help of these bundles one can better understand the geometry of the manifold $M$. A classical nontrivial vector bundle is the Möbius strip. This is a one-dimensional vector bundle over the circle $S^{1}$.

A first question which comes to mind is: how many vector bundles or better isomorphism classes of vector bundles are there on a manifold $M$ ? If one fixes the dimension of the vector bundles in question, there is a classifying space $B G L(n, \mathbb{C})$, which classifies isomorphism classes of complex vector bundles of dimension $n$.

If one wants to work with an algebraic variety, or more generally a scheme $X$ over an algebraically closed field $k$, one can also define vector bundles on $X$. But since a scheme $X$ comes with a sheaf of rings $\mathcal{O}_{X}$, we rather want to work with modules over $\mathcal{O}_{X}$. Fortunately there is a one-to-one correspondence:

\{isomorphism classes of vector bundles of dimension $n$ over $X$ \}

\{isomorphism classes of locally free sheaves of rank $n$ on $X$ \} .

So from now on we will work with locally free sheaves of rank $n$ on a scheme $X$.

In algebraic geometry there is no immediate classifying space which classifies locally free sheaves of rank $n$ on a scheme $X$. And in fact there is a long history in the classification problem for locally free sheaves on a scheme $X$.

One of the first results in this direction was a theorem due to Grothendieck in 1957, which states that if we have a locally free sheaf $F$ of rank $n$ on $X=\mathbb{P}^{1}$, then there are $n$ uniquely determined locally free sheaves of rank 1 , these are also called line bundles on $\mathbb{P}^{1}$, such that $F$ is the direct sum of these line bundles, that is:

$$
F \cong L_{1} \oplus \ldots \oplus L_{n}
$$

The next big step was done by Atiyah, also in 1957, who proved that isomorphism classes of indecomposable locally free sheaves of rank $n$ and degree $d$ on an elliptic curve $E$ are classified by $E$ itself.

But no general method to classify locally free sheaves on a curve $C$, let alone on a higher dimensional scheme $X$, was within sight.

The picture changed drastically in 1965, when Mumford introduced geometric invariant theory in [MF82], short GIT. This is a method that constructs quotients of a scheme $X$ by group actions in algebraic geometry. GIT is based on a paper of Hilbert from 1893 about classical invariant theory.

The modern view on classification problems in algebraic geometry is via functors on certain categories. Assume one wants to classify isomorphism classes of some structure. Then one defines a functor, the so-called moduli functor associated to the classification problem:

$$
\mathcal{M}: \mathrm{Sch}_{k} \longrightarrow \text { Sets, }
$$

here $S c h_{k}$ is the category of schemes over $k$ and Sets is the category of sets. This functor sends a scheme $S$ to the set of isomorphism classes of families of such structures over $S$. 
One says that a $k$-scheme $M$ is a fine moduli space for $\mathcal{M}$ if $M$ represents the functor $\mathcal{M}$, meaning there is a natural isomorphism $\mathcal{M} \cong \operatorname{Hom}(M,-)$ between functors.

Often a moduli functor is not representable. In this case one can weaken the notion of representability and still get a scheme $M$ which classifies the structures in question. Such a space is called a coarse moduli scheme.

Fine and coarse moduli schemes have the property that there is a one-to-one correspondece between the isomorphism classes of the structures one wants to classify and points in the space $M$. So in our situation we get moduli spaces of locally free sheaves of rank $n$ on a scheme $X$. This space is a replacement for the classifying space $B G L(n, \mathbb{C})$.

One hopes that by understanding the geometrical or topological properties of a moduli space $M$, one also gets a better understanding of the structures which are being classified by $M$.

For the construction of a moduli space of locally free sheaves of rank $n$ one uses Mumford's GIT. Usually one also fixes some numerical data of the sheaves one wants to classify, for example the Hilbert polynomial or, like in our case, the Chern classes.

Unfortunately even with these fixed numerical data there are just too many locally free sheaves of rank $n$ on a scheme $X$ to expect for a reasonable moduli space $M$ to exist. Instead one has to restrict to a special class of locally free sheaves of rank $n$, the so-called stable locally free sheaves. One can drop the stability condition, but then the moduli space does not exist in the category of schemes over $k$, but rather as a so-called Artin stack.

Using Mumford's GIT one can in fact show that for any $n \in \mathbb{N}$ and any smooth projective scheme $X$ there is a moduli space of stable locally free sheaves of rank $n$ and fixed numerical data on $X$. Today there are many results about moduli spaces of stable locally free sheaves of rank $n$ on a projective curve. But already for the case of projective surfaces the study of moduli spaces of stable locally free sheaves of rank two is really hard, see for example the book [Fri98]. More general results are known if the surface $X$ is a $K 3$ surface. Mukai showed in [Muk87] that the moduli spaces are always smooth in this case and that they admit a symplectic structure.

One example that is very well understood, on projective schemes of any dimension, is that of line bundles. These moduli spaces are called Picard schemes.

Another classical example of a moduli space is the moduli space $M_{g}$ of algebraic curves of genus $g$. It is known for example that this space has dimension $3 g-3$ if $g \geq 2$. If $g=1$, that is for elliptic curves, it is well known that $\mathbb{A}^{1}$ classifies isomorphism classes of such curves.

In this thesis we want to connect the ideas of moduli spaces of stable locally free sheaves of rank $n$ on a smooth projective surface $X$ for $n>1$ and that of Picard schemes.

To do this we define a special sheaf of algebras $\mathcal{A}$ on $X$, a so-called order on $X$. An order $\mathcal{A}$ on a smooth projective surface is a sheaf of associative $\mathcal{O}_{X}$-algebras, such that the stalk $\mathcal{A}_{\eta}$ at the generic point $\eta \in X$ is a division ring, which is finite dimensional over its center $k(X)$, the function field of $X$.

We want to study locally free sheaves on which the algebra $\mathcal{A}$ acts. We demand that the stalk at the generic point is a one-dimensional module over the generic stalk $\mathcal{A}_{\eta}$. This property is a substitution for stability. By this definition an $\mathcal{A}$-module $M$ can be seen as a module of rank one over $\mathcal{A}$, an $\mathcal{A}$-line bundle.

But as a locally free sheaf on $X$ such modules have the same rank as $\mathcal{A}$. In our example this rank is always a square number $r^{2}$ for some $r>1$, so that we work with locally free sheaves of arbitrary high rank on $X$. 
This thesis consits of five chapters:

- In the first chapter we define all objects that are involved: the algebras $\mathcal{A}$ we want to use and the $\mathcal{A}$-modules that are of interest to us. We will define the moduli functor which classifies these modules and see that it has a coarse moduli space $M_{\mathcal{A} / X ; c_{1}, c_{2}}$ of rank one $\mathcal{A}$-modules with fixed Chern classes. Then we will outline the theory of the so-called noncommutative cyclic covers. This is a theory which produces a lot of explicit examples of algebras $\mathcal{A}$. Following this we will collect and prove some facts about global Ext-groups and local $\mathcal{E} x t$-sheaves, for example we will show that there is a version of Serre duality for $\mathcal{A}$-modules. All these facts are generalizations of the appropriate situations for $\mathcal{O}_{X^{-}}$ modules. We will define the notion of a relative $\mathcal{E} x t$-sheaf for a morphism of schemes and as an application we will show that these relative $\mathcal{E} x t$-sheaves satisfy some kind of base change theorem. Furthermore we will gather some formulas concerning the Chern classes of the $\mathcal{A}$-modules we want to study. Finally, we will show that quasi-universal families, that is families that are classified by the moduli space itself, exist as well in our situation.

- In the second chapter we will introduce the notion of a so-called Mukai vector for $\mathcal{A}$ modules. Using this we can investigate moduli spaces of $\mathcal{A}$-modules on $K 3$ or abelian surfaces of low dimension, especially the moduli spaces of dimension zero and two. We will construct an explicit example of an algebra $\mathcal{A}$ on a product of two elliptic curves and study moduli spaces of bundles over this algebra.

- The third chapter is composed of more general results on moduli spaces of $\mathcal{A}$-modules on the projective plane $\mathbb{P}^{2}$. Especially we will prove that they are smooth for a certain kind of algebra $\mathcal{A}$, a so-called del Pezzo order. Then we will go on and study the deformation theory of the moduli spaces $M_{\mathcal{A} / X ; c_{1}, c_{2}}$. That is we are interested in how these bundles behave in families of $\mathcal{A}$-modules. As a consequence we can prove that if we have one non-empty moduli space we can construct infinitely many other non-empty moduli spaces, which are of their own interest.

- In the fourth and fifth chapter we recollect known explicit examples of moduli spaces and go on to construct algebras $\mathcal{A}$ on $\mathbb{P}^{2}$ of rank four respectively nine by using the theory of noncommutative cyclic covers. Then we will study explicit moduli spaces of $\mathcal{A}$-modules in these cases. These are by construction moduli spaces of locally free sheaves of rank four respectively nine. The last two chapters contain a lot of classical geometry, for example the 27 lines on a cubic surface will arise in the construction of the rank nine algebra.

- There is an appendix containing informations about Azumaya algebras and Brauer groups for the reader who is not that familiar with these concepts. Also there is a short section about global dimension for a sheaf of algebras. Furthermore we recollect some informations about the Grothendieck spectral sequence and prove a local-to-global spectral sequence for $\mathcal{A}$-modules. 
I would like to thank my supervisor Ulrich Stuhler for always having the time to discuss the various topics in this thesis. Without these discussions, this thesis would not have been possible.

I would also like to thank Viktor Pidstrygach for pointing out an error in the last chapter to me. 


\section{Contents}

Introduction $\quad 3$

$\begin{array}{ll}\text { Table of contents } & 7\end{array}$

1 Basics 9

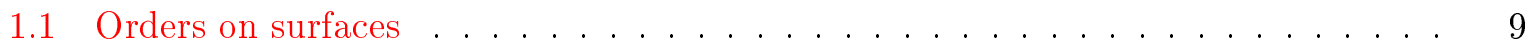

1.2 Modules over orders and moduli spaces . . . . . . . . . . . . . . . . . 14

1.3 Noncommutative cyclic covers . . . . . . . . . . . . . . . . . . . . . . . 16

1.4 Hom and Ext for modules over orders . . . . . . . . . . . . . . . . . . 19

1.5 Relative Ext-sheaves for modules over an Azumaya algebra . . . . . . . . . . 25

1.6 Chern class computations . . . . . . . . . . . . . . . . . 33

1.7 Quasi-universal families . . . . . . . . . . . . . . . . . . . . 38

2 Moduli spaces over K3 and abelian surfaces 41

2.1 Euler characteristic and Mukai vectors for modules over orders . . . . . . . . . . 42

2.2 Zero-dimensional moduli spaces . . . . . . . . . . . . . . . . . 46

2.3 Two-dimensional moduli spaces . . . . . . . . . . . . . . . . . 46

2.4 The case of an Azumaya Algebra on an abelian surface . . . . . . . . . . . . . 48

3 Moduli spaces over the projective plane: del Pezzo orders $\quad 55$

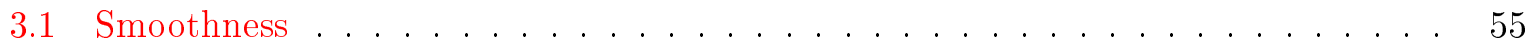

3.2 Punctual Quot-Schemes . . . . . . . . . . . . . . . . 58

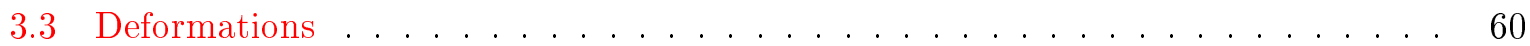

4 Examples of del Pezzo orders on the projective plane $\quad 71$

4.1 Del Pezzo order ramified on a smooth quartic . . . . . . . . . . . . . . . 71

4.2 Del Pezzo order ramified on two conics . . . . . . . . . . . . . . . . 73

4.3 Del Pezzo orders ramified on a smooth cubic curve . . . . . . . . . . . . . 75

4.3.1 Construction of an order . . . . . . . . . . . . . . . . . . . 79

4.3.2 Possible Chern classes . . . . . . . . . . . . . . . . . . . . . . . . 79

4.3.3 Moduli spaces of line bundles with minimal second Chern classes . . . . . 82

5 Moduli spaces on the projective plane: Calabi-Yau orders $\quad \mathbf{8 5}$

5.1 Construction of an order and some cohomological computations . . . . . . . . 85

5.2 Possible Chern classes . . . . . . . . . . . . . . . . . . . . 87

5.3 Moduli spaces of line bundles for minimal second Chern class . . . . . . . . . . . 89

$\begin{array}{ll}\text { A Azumaya algebras and Brauer groups } & 91\end{array}$

B Global dimension $\quad 92$

$\begin{array}{ll}\text { C Grothendieck spectral sequence } & 94\end{array}$

C.1 Local-to-global spectral sequence . . . . . . . . . . . . . . . . . . . . . 94

$\begin{array}{ll}\text { References } & 97\end{array}$ 


\section{Basics}

\subsection{Orders on surfaces}

We start by defining the algebras we are interested in. We will always assume, if not stated otherwise, that $k$ is an algebraically closed field of characteristic zero.

\section{Definition 1.1:}

Let $X$ be a smooth projective surface. An order $\mathcal{A}$ on $X$ is sheaf of associative $\mathcal{O}_{X}$-algebras with the following properties:

1. $\mathcal{A}$ is coherent and torsion-free as a sheaf of $\mathcal{O}_{X}$-modules;

2. The stalk $\mathcal{A}_{\eta}$ at the generic point $\eta \in X$ is a central division ring over the function field $k(X)=\mathcal{O}_{X, \eta}$ of $X$.

\section{Remark 1.2:}

If $\mathcal{A}$ is an order on $X$, then $X$ is sometimes called the center of $\mathcal{A}$, since $k(X)$ is the center of $\mathcal{A}_{\eta}$. Because a surface $X$ has dimension two, we will call orders on $X$ two-dimensional orders.

We can now look at all orders in $\mathcal{A}_{\eta}$ and order them by inclusion. A maximal element will be called a maximal order. These are the algebras we are interested in. Maximal orders have some nice properties, for example:

Lemma 1.3 ([Tan81, Proposition 6.3]):

Assume $\mathcal{A}$ is a maximal order on a smooth projective surface $X$, then $\mathcal{A}$ is a locally free $\mathcal{O}_{X}$ module.

Furthermore it is well known, that there is an open subset $U \subset X$ on which $\mathcal{A}$ is even an Azumaya algebra, see for example [Tan81, Proposition 6.2]. The complement $D:=X \backslash U$ is called the ramification locus of $\mathcal{A}$ and it is the union of finitely many curves $C \subset X$ and contains valuable informations about the order $\mathcal{A}$.

The ramification of a maximal order $\mathcal{A}$ can be read from the Artin-Mumford sequence:

Theorem 1.4 ([Tan81, Lemma 4.1]):

Let $X$ be a smooth projective surface, then there is a canonical exact sequence

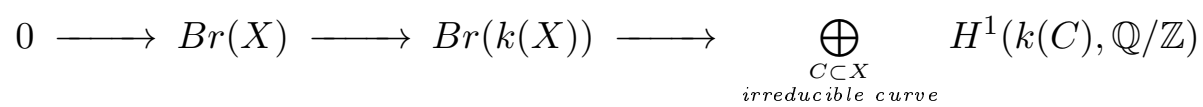

Here the Galois cohomology group $H^{1}(k(C), \mathbb{Q} / \mathbb{Z})$ classifies isomorphism classes of cyclic extensions of $k(C)$. More information about Azumaya algebras and Brauer groups can be found in the appendix.

The ramification curves are exactly the curves where the Brauer class of $\mathcal{A}_{\eta}$ has nontrivial image in $H^{1}(k(C), \mathbb{Q} / \mathbb{Z})$.

Thus every ramification curve $C$ comes with a finite cyclic field extension $L / k(C)$ and the degree $e_{C}:=[L: k(C)]$ is called the ramification index of $\mathcal{A}$ at $C$. This field extension also defines a cyclic cover $\tilde{C}$ of the normalization of $C$. Let $\tilde{D}$ be the disjoint union of the covers. 


\section{Remark 1.5:}

We remark that there is no maximal order $\mathcal{A}$ on $\mathbb{P}^{2}$ ramified only in a curve of degree one or two. This can be seen for degree one by using the so-called secondary ramification. Basically in this case this is due to the fact that $\mathbb{P}^{1}$ is simply connected, that is it has no nontrivial étale covers. If the degree of $D$ is two, then either $D$ is smooth and hence isomorphic to $\mathbb{P}^{1}$, or it is the union of two lines where a similiar argument applies.

This class of algebras is still too wide. We have no control over their homological properties, for example their global dimension (see (B.7) for the definition of the global dimension of $\mathcal{A}$ ). So we put some restraints on the ramification curves and ramification indices, which give maximal orders with reasonable properties.

Definition 1.6 ([CI05, Definition 2.5]):

A maximal order $\mathcal{A}$ on a smooth projective surface $X$ is called terminal if

- $D$ is a normal crossing divisor,

- the cyclic covers $\tilde{C}$ ramify only at the nodes of $D$,

- at a node $p$ one cover $\tilde{C}_{1}$ is totally ramified at $p$ of degree e and the other cover $\tilde{C}_{2}$ ramifies at $p$ with index $e$ and has degree ne for some $n \geq 1$.

If $\mathcal{A}$ is an Azumaya algebra on $X$, then it is known that the complete local structure is relatively easy. If $p \in X$ is a closed point and $\hat{\mathcal{O}}_{X, p}$ the complete local ring at $p$, then there is an isomorphism

$$
\mathcal{A}_{p} \otimes_{\mathcal{O}_{X, p}} \hat{\mathcal{O}}_{X, p} \cong M_{r}\left(\hat{\mathcal{O}}_{X, p}\right)
$$

where $r k(\mathcal{A})=r^{2}$.

One can ask if the complete local structure of a terminal order $\mathcal{A}$ at a closed point $p \in X$ can also be described. This was done in [CI05], where the authors also prove a minimal model program for orders on surfaces.

To describe the complete local structure of a terminal order $\mathcal{A}$ at a closed point $p \in X$, we identify the complete local ring $\hat{\mathcal{O}}_{X, p}$ with $R=k[[u, v]]$ appropriately and define

$$
S:=R<x, y>\text { with the relations } x^{e}=u, y^{e}=v \text { and } y x=\zeta x y
$$

where $e \in \mathbb{N}$ and $\zeta$ is a primitive $e$-th root of unity. Then $S$ if of finite rank over $R, Z(S)=R$ and $K(S)=S \otimes_{R} K$ is a division ring, here $K$ denotes the field of fractions $Q u o t(R)$ of $R$.

The following theorem describes the complete local structure:

Theorem 1.7 ([CI05, 2.3]):

Let $\mathcal{A}$ be a terminal maximal order and $p \in X$ be a closed point. Then there is an $\hat{\mathcal{O}}_{X, p}$-algebra isomorphism between $\mathcal{A} \otimes_{\mathcal{O}_{X}} \hat{\mathcal{O}}_{X, p}$ and a full matrix algebra over

$$
\left(\begin{array}{cccc}
S & \cdots & \cdots & S \\
x S & S & \ddots & \vdots \\
\vdots & \ddots & \ddots & \vdots \\
x S & \cdots & x S & S
\end{array}\right) \subset M_{n}(S)
$$


Knowing the complete local structure we can say even more:

Theorem 1.8 ([CI05, Proposition 2.8]):

Identifying $A:=\mathcal{A} \otimes \mathcal{O}_{X} \hat{\mathcal{O}}_{X, p}$ with the algebra described in (1.7) we have:

- A has global dimension two;

- if $n=e=1$, then $A$ is unramified;

- if $e=1$ and $n>1$ then $A$ is ramified on $u=0$ with ramification index $n$ and the cyclic cover $\tilde{D}$ of $D$ is unramified with degree $n$;

- if $e>1$ then $A$ is ramified on $u v=0$, the cyclic cover of $u=0$ has degree ne, the cyclic cover of $v=0$ has degree $e$ and both are ramified with ramification index $e$.

\section{Remark 1.9:}

If $p \in X$ is a smooth point of the ramification divisor $D$, then the complete local form simplifies to a matrix algebra over:

$$
B=\left(\begin{array}{cccc}
R & \cdots & \cdots & R \\
u R & R & \ddots & \vdots \\
\vdots & \ddots & \ddots & \vdots \\
u R & \cdots & u R & R
\end{array}\right)
$$

More exactly, the displayed ring $B$ lives in $M_{e}(R)$, where $e$ is the ramification index of $\mathcal{A}$ over the curve $C$ containing $p$. Then we have $A=M_{f}(B)$ for some $f \geq 1$.

\section{Remark 1.10:}

As a special case we note that a maximal order $\mathcal{A}$ with a smooth ramification locus is terminal.

Let $R$ be any commutative ring.

\section{Definition 1.11:}

An $R$-algebra $A$ is called Noetherian $R$-algebra if $R$ is Noetherian and $A$ is a finitely generated $R$-module.

Now if $M$ is an $A$-module, then it is also an $R$-module, via $R \rightarrow A$. This means we can compare properties of an $A$-module, when viewed as an $A$-module or as an $R$-module.

Lemma 1.12 ([Aus78, Proposition 4.2]):

Assume $A$ is a Noetherian R-algebra and let $M$ and $N$ be A-modules. Then we have the following comparison results:

- $M$ is a finitely generated A-module if and only if $M$ is a finitely generated $R$-module. (That is it we can talk about coherence conditions without reference to the base ring!)

- If $M$ and $N$ are finitely generated A-modules, then $\operatorname{Hom}_{A}(M, N)$ is a finitely generated $R$-module. 
- If $M$ is a finitely generated A-module, then $\operatorname{End}_{A}(M)$ is a Noetherian R-algebra.

- $M$ is of finite length over $A$ if and only if $M$ is of finite length over $R$.

Next we will list some useful lemmas about modules over orders in codimension one and two:

Lemma 1.13 ([Ram69, Proposition 3.5]):

Assume $R$ is a regular local ring of dimension $n$ and $A$ a Noetherian $R$-algebra, which is free as an $R$-module. Then $A$ has global dimension $n$ if and only if every finitely generated $R$-free A-module is A-projective.

Lemma 1.14 ([AG60, Proposition 2.2]):

Assume $R$ is a discrete valuation ring and $A$ is a maximal order, finitely generated and torsionfree as an $R$-module. If $M$ is an A-module, finitely generated and torsion-free as an $R$-module, then $M$ is A-projective.

Lemma 1.15 ([Ram69, Theorem 6.5(a)]):

Assume $R$ is an integrally closed Noetherian domain and suppose $A$ is a maximal $R$-order in $B:=A \otimes K$, where $K=Q u o t(R)$. If $M$ is a finitely generated $R$-reflexive left $A$-module, then $\operatorname{End}_{A}(M)$ is a maximal order in $\operatorname{End}_{B}(V)$, where $V:=M \otimes K$.

Lemma 1.16 ([AG60, Proposition 2.8]):

Assume $R$ is a Dedekind domain with $K=Q u o t(R)$. If $\Lambda$ is a hereditary $R$-algebra, which is a finitely generated and torsion-free $R$-module, then a finitely generated projective $\Lambda$-module $M$ is indecomposable if and only if $M \otimes_{R} K$ is a simple $\Lambda \otimes_{R} K$-module.

Lemma 1.17 ([AG60, Proposition 3.10]):

Assume $A$ is a maximal order over a discrete valuation ring $R$ and $M, N$ are two indecomposable A-modules, which are finitely generated and torsion-free over $R$. Then $M$ and $N$ are A-isomorphic.

Lemma 1.18 ([AG60, Proposition 3.7]):

Let $A$ be a maximal $R$-order in a simple algebra $B, R$ a discrete valuation ring. Then every indecomposable A-module, which is finitely generated and torsion-free over $R$, is a cyclic $A$ module. If $B$ is a division algebra, then every finitely generated projective A-module is free.

\section{Remark 1.19:}

A finitely generated and torsion-free module over a discrete valuation ring $R$ is free of finite rank. Since $R$ is a regular local ring of dimension one (1.14) implies that a maximal order, finitely generated and torsion-free as an $R$-module, has global dimension one by (1.13). In particular this means that if $\mathcal{A}$ is a maximal order on a smooth projective surface $X$, then for any point $\xi \in X$ of codimension one $\mathcal{A}_{\xi}$ has global dimension one. This especially implies that $\mathcal{A}_{\xi}$ is a hereditary, so that we can use (1.16).

Definition 1.20 ([CK03, Definition 4]):

Assume $\mathcal{A}$ is a terminal order on a smooth projective surface $X$. We define the canonical bimodule of $\mathcal{A}$ by: 


$$
\omega_{\mathcal{A}}=\mathcal{H o m}_{\mathcal{O}_{X}}\left(\mathcal{A}, \omega_{X}\right) .
$$

Here $\omega_{X}=\bigwedge^{2} \Omega_{X / k}$ is the canonical line bundle of $X$.

Definition 1.21 ([CK03, Lemma 8]):

Assume $\mathcal{A}$ is a terminal order on a smooth projective surface $X$ with ramification curves $\left\{C_{i}\right\}$ and ramification indicies $\left\{e_{i}\right\}$ for $i=1, \ldots, l$. Then we define the canonical divisor $K_{\mathcal{A}}$ of $\mathcal{A}$ by:

$$
K_{\mathcal{A}}=K_{X}+\sum_{i=1}^{l}\left(1-\frac{1}{e_{i}}\right) C_{i}
$$

Here $K_{X}$ is the canonical divisor of $X$, that is $\omega_{X}=\mathcal{O}_{X}\left(K_{X}\right)$.

Using the canonical divisor of a terminal order $\mathcal{A}$ we can define two classes of terminal orders, which will interest us the most. These are the so-called del Pezzo orders and Calabi-Yau orders.

\section{Definition 1.22:}

If $\mathcal{A}$ is a terminal order on a smooth projective surface $X$, then $\mathcal{A}$ is called del Pezzo order if $-K_{\mathcal{A}}$ is ample.

\section{Remark 1.23:}

Since $K_{\mathcal{A}}$ is a priori just a $\mathbb{Q}$-divisor, we need to define what ampleness means for $\mathbb{Q}$-divisors. If $D$ is a $\mathbb{Q}$-divisor, then $D$ is ample if and only if there is some $n \in \mathbb{N}$ such that $n D$ is an integral divisor and $n D$ is ample.

This definition relies on the fact that on a Noetherian scheme we have the following fact: a line bundle $L$ is ample if and only if $L^{m}$ is ample for some $m \in \mathbb{N}$, see [Har77, Proposition 7.5].

If $\mathcal{A}$ is a terminal del Pezzo order on $\mathbb{P}^{2}$, then its ramification is rather limited, due to the following proposition:

Proposition 1.24 ([CI05, Proposition 3.21]):

Assume $\mathcal{A}$ is a terminal del Pezzo order on $\mathbb{P}^{2}$ with ramification locus $D=\bigcup_{i=1}^{l} C_{i}$ and ramification indices $\left\{e_{i}\right\}_{1 \leq i \leq l}$. Then all ramification indices are equal and the degree of $D$ satifies:

$$
3 \leq \operatorname{deg}(D) \leq 5 .
$$

[[CI05, Proposition 3.21]] Furthermore there are more constraints for the ramification index $e \in \mathbb{N}$ depending on the degree of $D$.

\section{Lemma 1.25:}

Assume $\mathcal{A}$ is a terminal del Pezzo order on $\mathbb{P}^{2}$ with ramification locus $D=\bigcup_{i=1}^{l} C_{i}$ and ramification index $e$.

1. If $\operatorname{deg}(D)=3$ then any $e \geq 2$ is possible.

2. If $\operatorname{deg}(D)=4$ then $e=2$ or $e=3$ are possible.

3. If $\operatorname{deg}(D)=5$ then only $e=2$ is possible. 
If $\mathcal{A}$ is a terminal del Pezzo order on a smooth projective surface $X$, then $\mathcal{A}$ being del Pezzo also puts some constraints on $X$, like the next theorem shows:

Theorem 1.26 ([CK03, Theorem 12]):

Assume $\mathcal{A}$ is a terminal del Pezzo order on a smooth projective surface $X$. Then $X$ is a del Pezzo surface.

Del Pezzo orders are of special interest to us, because the moduli spaces of $\mathcal{A}$-modules we want to consider are automatically smooth in this case, see (3.6).

\section{Definition 1.27:}

If $\mathcal{A}$ is a terminal order on a smooth projective surface $X$, then $\mathcal{A}$ is called numerically Calabi-Yau if $K_{\mathcal{A}}$ is numerically trivial. We will call these orders just Calabi-Yau orders.

\section{Remark 1.28:}

Here a $\mathbb{Q}$-divisor $D$ is numerically trivial, $D \equiv 0$, if $D . C=0$ for every curve $C \subset X$.

\section{Lemma 1.29 ([CK05]):}

Assume $\mathcal{A}$ is a terminal Calabi-Yau order on a smooth projective surface $X$, then $X$ must be one of the following surfaces:

1. the blow up of $\mathbb{P}^{2}$ at at most 9 points in almost general position, $\mathbb{P}^{1} \times \mathbb{P}^{1}$ or the Hirzebruch surface $\mathbb{F}_{2}=\mathbb{P}(\mathcal{O} \oplus \mathcal{O}(-2))$;

2. a ruled surface $\mathbb{P}(\mathcal{E}) \rightarrow C, C$ an elliptic and either $\mathcal{E}=\mathcal{O} \oplus \mathcal{L}$ with $\mathcal{L}^{n}=\mathcal{O}$ for $n \in$ $\{1,2,3,4\}$ or $\mathcal{E}$ is indecomposable of degree one;

3. a surface of Kodaira dimension zero.

\section{Remark 1.30:}

In the last case of the previous lemma, the order $\mathcal{A}$ must be unramified. Hence it must be an Azumaya algebra, see (2.4) for an example of a Calabi-Yau order on a surface with Kodaira dimension zero. In (5.1) we will see an example of a Calabi-Yau order on $\mathbb{P}^{2}$. Like in the case of del Pezzo orders one can classify the possible ramification data for terminal Calabi-Yau orders, see [CK05] for more information.

\subsection{Modules over orders and moduli spaces}

Let $X$ be a smooth projective surface and let $\mathcal{A}$ be a terminal order on $X$. Now we want to study modules over $\mathcal{A}$ and their moduli spaces.

\section{Definition 1.31:}

If $M$ is a left $\mathcal{A}$-module, then we call $M$ a torsion-free $\mathcal{A}$-module of rank one if:

1. as a sheaf of $\mathcal{O}_{X}$-modules $M$ is coherent and torsion-free;

2. the stalk $M_{\eta}$ over the generic point $\eta \in X$ has dimension one over the division ring $\mathcal{A}_{\eta}$. 
To study moduli spaces one needs to have the notion of a family of $\mathcal{A}$-modules. To define this we need to understand how $\mathcal{A}$ behaves under base change, so let $k \subset K$ be a field extension and let $\mathcal{A}_{K}$ be the pullback of $\mathcal{A}$ to $X_{K}:=X \times_{k} \operatorname{Spec}(K)$. Then we have the following lemma:

Lemma 1.32 ([HS05, Lemma 1.2]):

The generic stalk of $\mathcal{A}_{K}$ is a central division ring over the function field of $X_{K}$.

\section{Definition 1.33:}

A family of torsion-free $\mathcal{A}$-modules of rank one over a $k$-scheme $S$ is a sheaf $\mathcal{F}$ of left $\mathcal{A}_{S}$-modules, where $\mathcal{A}_{S}$ is the pullback of $\mathcal{A}$ to $X \times_{k} S$, with the following properties:

1. $\mathcal{F}$ is coherent over $\mathcal{O}_{X \times_{k} S}$ and flat over $S$;

2. for every $s \in S$, the fiber $\mathcal{F}_{s}$ is a torsion-free $\mathcal{A}_{k(s)}$-module of rank one.

Here $k(s)$ is the residue field of $S$ at $s$ and the fiber is the pullback of $\mathcal{F}$ to $X \times_{k} \operatorname{Spec}(k(s))$.

Now one can define the following moduli functor:

$$
\mathcal{M}_{\mathcal{A} / X}: \text { Sch }_{k} \longrightarrow \text { Sets }
$$

which sends a $k$-scheme $S$ to the set of isomorphism classes of families $\mathcal{F}$ of torsion-free $\mathcal{A}$ modules of rank one over $S$. The functor classifying modules with fixed Hilbert polynomial $P$ is denoted by $\mathcal{M}_{\mathcal{A} / X ; P}$.

Theorem 1.34 ([HS05, Theorem 2.4]):

There is a coarse moduli scheme $M_{\mathcal{A} / X ; P}$ for the functor $\mathcal{M}_{\mathcal{A} / X ; P} . M_{\mathcal{A} / X ; P}$ is a projective scheme of finite type over $k$.

So there also exists a coarse moduli scheme for $\mathcal{M}_{\mathcal{A} / X}$ :

$$
M_{\mathcal{A} / X}=\coprod_{P} M_{\mathcal{A} / X ; P} .
$$

There is another decomposition for $M_{\mathcal{A} / X}$ by fixing the Chern classes, for a smooth projective surface $X$ this reads:

$$
M_{\mathcal{A} / X}=\coprod_{\substack{c_{1} \in N S(X) \\ c_{2} \in \mathbb{Z}}} M_{\mathcal{A} / X ; c_{1}, c_{2}} .
$$

We will work with the spaces with fixed Chern classes.

\section{Remark 1.35:}

Similar spaces have been considered by Lieblich and Yoshioka, see for example [Yos06] and [Lie07].

If we fix the first Chern class of the modules in question, the second Chern class can still vary. If these numbers in $\mathbb{Z}$ are bounded from below, then one can choose a minimal second Chern class. The moduli spaces with minimal second Chern classes are of special interest and will be denoted by $\operatorname{Pic}(\mathcal{A})_{c_{1}, c_{2}}$ or, if no confusion arises, just by $\operatorname{Pic}(\mathcal{A})$. The reason is the following: 


\section{Lemma 1.36:}

If for fixed first Chern class $c_{1}$ one can choose a minimal second Chern class $c_{2}$, then all modules classified by $M_{\mathcal{A} / X ; c_{1}, c_{2}}$ are actually locally projective $\mathcal{A}$-modules of rank one.

\section{Proof:}

Assume $M$ has Chern classes as described and $M$ is not a locally projective, but just a torsion-free $\mathcal{A}$-module of rank one. Then there is a canonical exact sequence

$$
0 \longrightarrow M \longrightarrow M^{* *} \longrightarrow T \longrightarrow 0
$$

with $M^{* *}$ the bidual of $M$, hence $c_{1}(M)=c_{1}\left(M^{* *}\right)$, and the quotient $T$ is an Artinian sheaf of finite length, since $M$ is torsion-free. But then $c_{1}(T)=0$ and $c_{2}(T)<0$ which implies that

$$
c_{2}\left(M^{* *}\right)=c_{2}(M)+c_{2}(T)<c_{2}(M) .
$$

But $M^{* *}$ is also an $\mathcal{A}$-module and the second Chern class of $M$ is the minimal one, so the assumption was wrong and $M \cong M^{* *}$. Since $M^{* *}$ is reflexive it is locally free on $X$, so by $(1.56$, still to come) it is a locally projective $\mathcal{A}$-module and so is $M$.

Lemma 1.37 ([CC11, Proposition 4.2]):

Assume $\mathcal{A}$ is a terminal order on a smooth projective surface $X$. If $M$ is a locally projective $\mathcal{A}$ module of rank one, then for every closed point $p \in X$ we have an isomorphism of $\mathcal{A}_{p}$-modules:

$$
M_{p} \cong \mathcal{A}_{p}
$$

\subsection{Noncommutative cyclic covers}

In this section we want to describe a method that gives us explicit examples of orders on surfaces with prescribed ramification data, the so-called noncommutative cyclic covering trick. For detailed information see [Cha05]. To do this we start with a smooth projective scheme $X$ and define the notion of an invertible $\mathcal{O}_{X}$-bimodule.

\section{Definition 1.38:}

An invertible bimodule on $X$ is of the form $L_{\sigma}$, where $L \in \operatorname{Pic}(X)$ and $\sigma \in$ Aut $(X)$. The bimodule $L_{\sigma}$ can be thought of as the $\mathcal{O}_{X}$-module $L$ where the left action is the usual one $\mathcal{O}_{X} L \cong L$ and the right action is twisted by the automorphism $\sigma$, that is $L_{\mathcal{O}_{X}} \cong \sigma^{*} L$.

Using this definition one can compute the tensor product of invertible modules using the following formula:

$$
L_{\sigma} \otimes M_{\tau}=\left(L \otimes \sigma^{*} M\right)_{\tau \sigma} .
$$

The bimodule $L_{\sigma}$ defines an auto-equivalence of $\operatorname{Coh}(X)$ by $L_{\sigma} \otimes(-):=L \otimes \sigma^{*}(-)$.

Using invertible bimodules one can define so-called cyclic algebras on $X$.

Let $\sigma \in \operatorname{Aut}(X)$ be an automorphism of finite order $e$, set $G=\left\langle\sigma \mid \sigma^{e}=1\right\rangle$ and pick $L \in \operatorname{Pic}(X)$. Assume $D$ is an effective Cartier divisor on $X$ and suppose there is an isomorphism of invertible bimodules

$$
\phi: L_{\sigma}^{e} \stackrel{\sim}{\longrightarrow} \mathcal{O}_{X}(-D) .
$$


Denote by $\phi$ also the morphism $\phi: L_{\sigma}^{e} \stackrel{\sim}{\longrightarrow} \mathcal{O}_{X}(-D) \hookrightarrow \mathcal{O}_{X}$ and consider it as a relation on the tensor algebra

$$
T\left(X, L_{\sigma}\right):=\bigoplus_{n \geq 0} L_{\sigma}^{n}
$$

We say that the relation $\phi$ satisfies the so-called overlap condition, if the following diagram commutes:

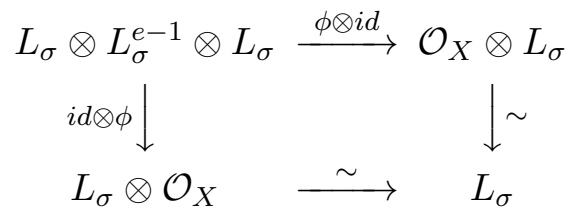

We define the cyclic algebra $A\left(X, L_{\sigma}, \phi\right)$ by:

$$
A\left(X, L_{\sigma}, \phi\right):=T\left(X, L_{\sigma}\right) /(\phi) .
$$

If $\phi$ satisfies the overlap condition, then one can show that:

$$
A\left(X, L_{\sigma}, \phi\right)=\bigoplus_{n=0}^{e-1} L_{\sigma}^{n}
$$

The multiplication on $A\left(X, L_{\sigma}, \phi\right)$ is induced by

$$
L_{\sigma}^{n} \otimes L_{\sigma}^{m} \longrightarrow \begin{cases}L_{\sigma}^{n+m} & n+m<e \\ L_{\sigma}^{n+m} \stackrel{\phi}{\rightarrow} L_{\sigma}^{n+m-e} & n+m \geq e .\end{cases}
$$

\section{Example 1.39:}

Assume $F$ is a field and set $X=\operatorname{Spec}(F)$. Pick an automorphism $\sigma \in A u t(F)$ of order $e$ and let $G$ be the cyclic group generated by $\sigma$. Now if $K$ denotes $F^{G}$, then $F / K$ is a cyclic extension of degree $e$.

An invertible bimodule $L_{\sigma}$ can be written as $F z$, such that we have $z a=\sigma(a) z$ for $a \in F$. The tensor powers are given by $L_{\sigma}^{n}=(F z)^{n}=F z^{n}$, where we have $z^{n} a=\sigma^{n}(a) z^{n}$.

Now suppose there is a relation $\phi: F z^{e} \longrightarrow F$, then $\phi$ is defined by multiplication with some element $b \in F$ such that $z^{e}=b$. Now the overlap condition is equivalent to $b z=z b$, which implies $\sigma(b)=b$ or $b \in F^{G}=K$.

The resulting cyclic algebra $A\left(X, L_{\sigma}, \phi\right)$ is the well known cyclic algebra $F[z, \sigma] /\left(z^{e}-b\right)$. Note that this algebra is a central simple $K$-algebra of K-dimension $e^{2}$.

\section{Example 1.40:}

We look at the previous example and take $F=\mathbb{C}$ and $\sigma=\overline{(~)}$ the complex conjugation on $\mathbb{C}$, thus $G=\mathbb{Z} / 2 \mathbb{Z}$. As the bimodule we pick $L_{\sigma}=\mathbb{C} j$ with $j^{2}=-1$.

Then we have $j r=r j$ for $r \in \mathbb{R}$ and $j \mathrm{i}=-\mathrm{i} j$ for $\mathrm{i} \in \mathbb{C}$. So we see that

$$
A\left(X, L_{\sigma}, \phi\right)=\mathbb{C}[j, \overline{(\quad)}] /\left(j^{2}+1\right)=\mathbb{C} \oplus \mathbb{C} j=\mathbb{R} \oplus \mathbb{R} \mathbf{i} \oplus \mathbb{R} j \oplus \mathbb{R} \mathrm{i} j .
$$

But the last algebra is known as the Hamiltonian quaternions $\mathbb{H}$.

We will be most interested in such examples where $D=0$, that is the relation $\phi: L_{\sigma}^{e} \stackrel{\sim}{\longrightarrow} \mathcal{O}_{X}$ is an isomorphism. In this cases there are some lemmas which are of interest to us: 
Lemma 1.41 ([BA12, Theorem 2.4]):

Assume $X$ and $Y$ are smooth projective surfaces, such that there is a cyclic cover $\pi: X \rightarrow Y$ of degree e with Galois group $G=\langle\sigma\rangle$. Let $A\left(X, L_{\sigma}, \phi\right)$ be a cyclic algebra coming from a relation of the form $\phi: L_{\sigma}^{e} \stackrel{\sim}{\longrightarrow} \mathcal{O}_{X}$. If $\phi$ satifies the overlap condition then $A\left(X, L_{\sigma}, \phi\right)$ defines an order $\mathcal{A}$ on $Y$ (via $\pi$ ) and its ramification over $C \subset Y$ is exactly the ramification of $\pi$ above $C$.

Lemma 1.42 ([BA12, Theorem 2.5]):

Assume $X$ and $Y$ are smooth projective surfaces, such that there is a cyclic cover $\pi: X \rightarrow Y$ of degree e, with Galois group $G=\langle\sigma\rangle$ and totally ramified at $D \subset X$. Consider the cyclic algebra $A\left(X, L_{\sigma}, \phi\right)$ coming from a relation of the form $\phi: L_{\sigma}^{e} \stackrel{\sim}{\longrightarrow} \mathcal{O}_{X}$. Then the ramification of $A\left(X, L_{\sigma}, \phi\right)$ along $\pi(D)$ is the cyclic cover of $D$ given by the e-torsion line bundle $L_{\mid D}$.

Lemma 1.43 ([BA12, Lemma 2.8]):

A cyclic algebra $A\left(X, L_{\sigma}, \phi\right)$ is a maximal order on $Y$ if for all irreducible components $C_{i}$ of the ramification divisor, the cover $\tilde{C}_{i}$ is irreducible.

We are interested in relations of the form $L_{\sigma}^{e} \stackrel{\sim}{\longrightarrow} \mathcal{O}_{X}$. Using the definition of the tensor product for bimodules, we see that $L_{\sigma}^{e}=L \otimes_{\mathcal{O}_{X}} \sigma^{*} L \otimes_{\mathcal{O}_{X}} \ldots \otimes_{\mathcal{O}_{X}}\left(\sigma^{e-1}\right)^{*} L$ since $\sigma^{e}=i d$ by definition. So if we consider $\operatorname{Pic}(X)$ as a $G$-Set for $G=\langle\sigma\rangle$, then we are looking for $L \in \operatorname{Pic}(X)$ such that $L \in \operatorname{ker}\left(1+\sigma+\ldots+\sigma^{e-1}\right)$. So these line bundles can be classified by using group cohomology. Since $G$ is cyclic, the cohomology of any $G$-Set $M$ can be read off the sequence

$$
\text { .. } \stackrel{N}{\longrightarrow} M \stackrel{D}{\longrightarrow} M \stackrel{N}{\longrightarrow} M \stackrel{D}{\longrightarrow} \ldots
$$

where $D=(1-\sigma)$ and $N=\left(1+\sigma+\ldots+\sigma^{e-1}\right)$. Now 1-cocylces of the $G$-set $\operatorname{Pic}(X)$ are exactly the line bundles with the desired relations. We will also write $L$ for the class of the line bundle $L$ in $H^{1}(G, \operatorname{Pic}(X))$. Here we have $H^{0}(G, \operatorname{Pic}(X))=\operatorname{ker}(D)=\operatorname{Pic}(X)^{G}$ and $H^{1}(G, \operatorname{Pic}(X))=\operatorname{ker}(N) / \operatorname{im}(D)$. Using the group cohomology we can now see when a certain relation satisfies the overlap condition.

Lemma 1.44 ([BA12, Proposition 2.10]):

Assume $X$ and $Y$ are smooth projective surfaces such that there is a cyclic cover $\pi: X \rightarrow Y$ of degree $e$ and the lowest common multiple of the ramification indices of $\pi$ is e. Then all relations created from elements of $H^{1}(G, P i c(X))$ satisfy the overlap condition.

Finally, we would like to know if the orders constructed via the noncommutative cyclic covering trick are generically nontrivial, meaning we want to know if their Brauer classes are nontrivial in $\operatorname{Br}(k(Y))$. Again using group cohomology this can be checked:

Lemma 1.45 ([Cha05, Corollary 4.4]):

Assume $X$ and $Y$ are smooth projective surfaces and suppose that there is cyclic cover $\pi: X \rightarrow Y$ of degree e, with Galois group $G=\langle\sigma\rangle$ and totally ramified at one irreducible divisor $D \subset X$. Suppose further that $D$ is not torsion in Pic $(X)$. Then there is a group monomorphism

$$
\Psi: H^{1}(G, \operatorname{Pic}(X)) \rightarrow \operatorname{Br}(k(X) / k(Y))
$$

given as follows: if $L \in \operatorname{Pic}(X)$ represents a 1-cocycle in $H^{1}(G, \operatorname{Pic}(X))$ then any relation $\phi: L_{\sigma}^{e} \stackrel{\sim}{\longrightarrow} \mathcal{O}_{X}$ satisfies the overlap condition and $\Psi(L)=k(Y) \otimes_{\mathcal{O}_{Y}} A\left(X, L_{\sigma}, \phi\right)$ in $\operatorname{Br}(k(Y))$. 
Here $\operatorname{Br}(k(X) / k(Y))=\operatorname{ker}\left(f: B r(k(Y)) \rightarrow B r(k(X))\right.$, where $f(A)=A \otimes_{k(Y)} k(X)$.

Lemma 1.46 ([CK11, Proposition 2.6]):

Assume $A$ is a cyclic algebra constructed via the noncommutative cyclic covering trick, that is $A=A\left(X, L_{\sigma}, \phi\right)$, then there is a natural isomorphism:

$$
\operatorname{Ext}_{A}^{p}\left(A \otimes_{X} N,-\right) \cong \operatorname{Ext}_{\mathcal{O}_{X}}^{p}(N,-)
$$

for all $p \geq 0$ and all coherent $\mathcal{O}_{X}$-modules $N$.

\subsection{Hom and Ext for modules over orders}

\section{Lemma 1.47:}

Assume $\mathcal{A}$ is an order on a smooth projective surface $X$. Let $M$ and $N$ be torsion-free $\mathcal{A}$-modules of rank one and $\phi \in \operatorname{Hom}_{\mathcal{A}}(M, N)$. If $\phi$ is nontrivial, then $\phi$ is injective.

\section{Proof:}

The given map induces the following short exact sequence:

$$
0 \longrightarrow \operatorname{ker}(\phi) \longrightarrow M \stackrel{\phi}{\longrightarrow} \operatorname{im}(\phi) \longrightarrow 0 \text {. }
$$

Since $\phi \neq 0$, we have $i m(\phi) \neq 0$. As $i m(\phi)$ is a subsheaf of the torsion-free sheaf $N$, it is itself torsion-free. This implies $i m(\phi)_{\eta} \neq 0$. Therefore $i m(\phi)_{\eta}$ is a nontrivial $\mathcal{A}_{\eta}$-submodule of $N_{\eta}$. Since $N_{\eta}$ is simple, we see that $i m(\phi)_{\eta} \cong N_{\eta}$. So $\phi_{\eta}$ is an isomorphism, which shows that $\operatorname{ker}(\phi)_{\eta}=0$. So $\operatorname{ker}(\phi)$ is a torsion sheaf, but as a subsheaf of the torsion-free sheaf $M$ it is torsion-free. So it is torsion and torsion-free, which shows that it must be zero: $\operatorname{ker}(\phi)=0$.

\section{Lemma 1.48:}

Assume $\mathcal{A}$ is an order on a smooth projective surface $X$. Let $M$ and $N$ be torsion-free $\mathcal{A}$ modules of rank one with the same Chern classes. If $\operatorname{Hom}_{\mathcal{A}}(M, N)$ is nontrivial, then $M$ and $N$ are isomorphic $\mathcal{A}$-modules.

\section{Proof:}

By (1.47) a nontrivial element in $\operatorname{Hom}_{\mathcal{A}}(M, N)$ gives rise to a short exact sequence:

$$
0 \longrightarrow \mathrm{M} \longrightarrow \mathrm{N} \longrightarrow \mathrm{T} \longrightarrow 0 .
$$

Since $M$ and $N$ are torsion-free $\mathcal{A}$-modules of rank one, the quotient $T$ is a torsion sheaf with $\operatorname{codim}(\operatorname{supp}(T)) \geq 1$.

Since $c_{1}(M)=c_{1}(N)$, we see that $c_{1}(T)=0$. Now by (1.80) we have

$$
c_{1}(T)=\sum_{\operatorname{codim}(\xi)=1} l_{\mathcal{O}_{X, \xi}}\left(T_{\xi}\right) \overline{\{\xi\}}
$$

and we see that we must have $l_{\mathcal{O}_{X, \xi}}\left(T_{\xi}\right)=0$ for all points of codimension one. But this implies $T_{\xi}=0$ for all points in codimension one, meaning $T$ has no support in codimension one. So $\operatorname{codim}(\operatorname{supp}(T)) \geq 2$.

Therefore the sheaf $T$ is an Artinian sheaf of finite length. Since $c_{2}(M)=c_{2}(N)$ and $c_{1}(T)=0$ we can see that $c_{2}(T)=0$ by using the exact sequence (1) and the properties of Chern classes on exact sequences.

But $c_{2}(T)=-\operatorname{dim}\left(H^{0}(X, T)\right)$ by Hirzebruch-Riemann-Roch, so $H^{0}(X, T)=0$.

As $T$ has finite length, we know that 


$$
H^{0}(X, T)=\bigoplus_{x \in \operatorname{supp}(T)} T_{x}
$$

This implies $T_{x}=0$ for all $x \in \operatorname{supp}(T)$. We conclude that $T=0$, or in other words, by the exact sequence (1): $M \cong N$.

\section{Lemma 1.49:}

Assume $\mathcal{A}$ is an order on a smooth projective surface $X$ and $M$ is a torsion-free $\mathcal{A}$-module of rank one, then $M$ is simple, that is $\operatorname{End}_{\mathcal{A}}(M)=k$.

\section{Proof:}

As $i d_{M} \in E n d_{\mathcal{A}}(M)$ we have $E n d_{\mathcal{A}}(M) \neq\{0\}$. Because $M$ is coherent, we see that $\operatorname{End_{\mathcal {A}}(M)}$ is a finite dimensional $k$-algebra since $\operatorname{End} d_{\mathcal{A}}(M)=H^{0}\left(X, \mathcal{E} n d_{\mathcal{A}}(M)\right)$.

The sheaf $\mathcal{E} n d_{\mathcal{A}}(M)$ is given at the generic point $\eta$ of $X$ by $\mathcal{E} n d_{\mathcal{A}}(M)_{\eta}=\operatorname{End}_{\mathcal{A}_{\eta}}\left(M_{\eta}\right) \cong \mathcal{A}_{\eta}^{o p}$. So $\operatorname{End}_{\mathcal{A}}(M)$ embeds into the division ring $\mathcal{A}_{\eta}^{o p}$.

This shows that $\operatorname{End}_{\mathcal{A}}(M)$ is a finite dimensional $k$-algebra without zero divisors, hence it is a division algebra over $k$. Since $k$ is algebraically closed this algebra must be $k$ itself.

\section{Remark 1.50:}

Considering $\mathcal{A}$ as a torsion-free $\mathcal{A}$-module of rank one, the previous lemma (1.49) shows:

$$
H^{0}(X, \mathcal{A})=\operatorname{End}_{\mathcal{A}}(\mathcal{A})=k
$$

for every order $\mathcal{A}$ on $X$.

To understand the $E x t_{\mathcal{A}^{-}}^{i}$ groups, we need an analogue of the local-to-global spectral sequence in the case of $\mathcal{O}_{X}$-modules. See Appendix (C.10) for a proof of the following theorem.

\section{Theorem 1.51:}

Assume $\mathcal{A}$ is an order on a smooth projective surface $X$ and let $M$ and $N$ be $\mathcal{A}$-modules, then there is a local-to-global spectral sequence:

$$
E_{2}^{p, q}=H^{p}\left(X, \mathcal{E} x t_{\mathcal{A}}^{q}(M, N)\right) \Rightarrow \operatorname{Ext}_{\mathcal{A}}^{p+q}(M, N)
$$

\section{Lemma 1.52:}

Assume $\mathcal{A}$ is a terminal order on a smooth projective surface $X$ and let $M$ and $N$ be two coherent $\mathcal{A}$-modules. Then $\operatorname{Ext}_{\mathcal{A}}^{i}(M, N)=0$ for all $i \geq 3$.

\section{Proof:}

Since $\mathcal{A}$ has global dimension two and $M$ and $N$ are coherent, we see that the local $\mathcal{E} x t$-sheaves $\mathcal{E}_{x} t_{\mathcal{A}}^{i}(M, N)$ vanish for $i \geq 3$. Using the local-to-global spectral sequence, it is enough to show that:

1. $H^{2}\left(X, \mathcal{E} x t_{\mathcal{A}}^{i}(M, N)\right)=0$ for $i=1,2$

2. $H^{1}\left(X, \mathcal{E} x t_{\mathcal{A}}^{2}(M, N)\right)=0$. 
This is because these are the only modules who contribute to $E x t_{\mathcal{A}^{-}}^{i}$ groups for $i \geq 3$.

Since $\mathcal{A}$ is a division ring at the generic point $\eta$, every module over $\mathcal{A}_{\eta}$ is projective, which shows that for all $i \geq 1$ we have $\operatorname{Ext}_{\mathcal{A}_{\eta}}^{i}\left(M_{\eta}, N_{\eta}\right)=0$. So $\operatorname{dim}\left(\operatorname{supp}\left(\mathcal{E} x t_{\mathcal{A}}^{i}(M, N)\right)\right) \leq 1$ for $i=1,2$. This immediately implies $\left.H^{2}\left(X, \mathcal{E} x t_{\mathcal{A}}^{i}(M, N)\right)\right)=0$ for $i=1,2$.

If $\xi$ is a point of codimension one, then $\mathcal{A}_{\xi}$ has global dimension one, so that $\operatorname{Ext}_{\mathcal{A}_{\xi}}^{2}\left(M_{\xi}, N_{\xi}\right)=0$. This implies $\operatorname{dim}\left(\operatorname{supp}\left(\mathcal{E} x t_{\mathcal{A}}^{2}(M, N)\right)\right)=0$, which shows that $H^{1}\left(X, \mathcal{E} x t_{\mathcal{A}}^{2}(M, N)\right)=0$.

\section{Lemma 1.53:}

Assume $\mathcal{A}$ is a maximal order on a smooth projective surface and let $M$ and $N$ be $\mathcal{A}$-modules. If $M$ is a coherent locally projective $\mathcal{A}$-module, then there is an isomorphism:

$$
\mathcal{A}^{*} \otimes_{\mathcal{O}_{X}} \mathcal{H o m} \mathcal{A}_{\mathcal{A}}(M, N) \cong \mathcal{H o m}_{\mathcal{O}_{X}}(M, N) .
$$

\section{Proof:}

Writing $\mathcal{A}^{*}=\mathcal{H} \mathrm{m}_{\mathcal{O}_{X}}\left(\mathcal{A}, \mathcal{O}_{X}\right)$ we see there is a natural map

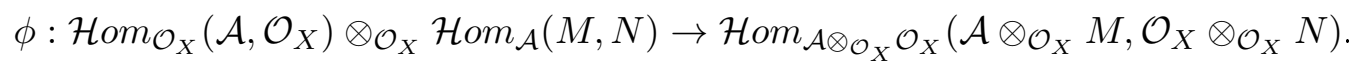

We can check at the stalk level if this map is an isomorphism. So let $p \in X$ be any point, then we have the following situation:

$R=\mathcal{O}_{X, p}$ is a commutative local ring and $A=\mathcal{A}_{p}$ is an $R$-algebra, free of finite rank as an $R$-module. $M_{p}$ is a finitely generated projective $A$-module and $N_{p}$ is an $A$-module, and we have to show that the map

$$
\operatorname{Hom}_{R}(A, R) \otimes_{R} \operatorname{Hom}_{A}\left(M_{p}, N_{p}\right) \rightarrow \operatorname{Hom}_{R \otimes_{R} A}\left(A \otimes_{R} M_{p}, R \otimes_{R} N_{p}\right)
$$

is an isomorphism. But this is true, even in more generality, see for example [DI71, I.2.4]. There is a canonical isomorphism

$$
\mathcal{H o m}_{\mathcal{A} \otimes_{\mathcal{O}_{X}} \mathcal{O}_{X}}\left(\mathcal{A} \otimes_{\mathcal{O}_{X}} M, \mathcal{O}_{X} \otimes_{\mathcal{O}_{X}} N\right) \cong \mathcal{H o m}_{\mathcal{A}}\left(\mathcal{A} \otimes_{\mathcal{O}_{X}} M, N\right) .
$$

Using the tensor-hom-adjunction we see that

$$
\mathcal{H o m}_{\mathcal{A}}\left(\mathcal{A} \otimes \mathcal{O}_{X} M, N\right)=\mathcal{H o m}_{\mathcal{O}_{X}}\left(M, \mathcal{H o m}{ }_{\mathcal{A}}(\mathcal{A}, N)\right) .
$$

Since $N$ is an $\mathcal{A}$-module $\mathcal{H o m}_{\mathcal{A}}(\mathcal{A}, N)=N$. Putting all together we see that there is an isomorphism

$$
\mathcal{A}^{*} \otimes_{\mathcal{O}_{X}} \mathcal{H o m}_{\mathcal{A}}(M, N) \cong \mathcal{H o m}_{\mathcal{O}_{X}}(M, N) .
$$

\section{Remark 1.54:}

If $\mathcal{A}$ is even an Azumaya algebra, then this result is true on any finite-dimensional smooth projective scheme. This is because Azumaya algebras are by definition coherent and locally free.

\section{Lemma 1.55:}

Assume $\mathcal{A}$ is a maximal order on a smooth projective surface $X$ and $M$ is a coherent $\mathcal{A}$-module. If $M$ is locally projective as an $\mathcal{A}$-module, then it is locally free as an $\mathcal{O}_{X}$-module 


\section{Proof:}

We will show that $\mathcal{H o m}_{\mathcal{O}_{X}}(M,-)$ is an exact functor. Using the $\mathcal{A}$-module structure and the tensor-hom-adjunction we see that

$$
\begin{aligned}
\mathcal{H o m}_{\mathcal{O}_{X}}(M,-) & =\mathcal{H o m}_{\mathcal{O}_{X}}\left(\mathcal{A} \otimes_{\mathcal{A}} M,-\right) \\
& =\mathcal{H o m}_{\mathcal{A}}\left(M, \mathcal{H o m}_{\mathcal{O}_{X}}(\mathcal{A},-)\right)
\end{aligned}
$$

But $\mathcal{A}$ is a coherent locally free $\mathcal{O}_{X}$-module, that is $\mathcal{H o m}_{\mathcal{O}_{X}}(\mathcal{A},-)$ is exact, and $M$ is a coherent locally projective $\mathcal{A}$-module, so $\mathcal{H o m}_{\mathcal{A}}(M,-)$ is exact. But then $\mathcal{H o m}_{\mathcal{O}_{X}}(M,-)$ must be exact, so $M$ is a locally free $\mathcal{O}_{X}$-module, see [Har77, Exercise III.6.5 (a)].

\section{Corollary 1.56:}

Assume $\mathcal{A}$ is a terminal order on a smooth projective surface $X$ and $M$ is a coherent $\mathcal{A}$-module. Then $M$ is locally projective as an $\mathcal{A}$-module if and only if $M$ is locally free as an $\mathcal{O}_{X}$-module.

\section{Proof:}

If $M$ is a locally projective $\mathcal{A}$-module, then by the previous lemma (1.55) $M$ is a locally free $\mathcal{O}_{X}$-module. Now if $M$ is an $\mathcal{A}$-module, locally free as an $\mathcal{O}_{X}$-module, the result follows from (1.13), since $M$ is coherent and $\mathcal{A}$ is locally free and has global dimension two.

\section{Remark 1.57:}

If $\mathcal{A}$ is an Azumaya algebra, then this result is true for any finite-dimensional smooth projective scheme. This is because, by [HS05, Proposition 3.4], an Azumaya algebra has global dimension $\operatorname{dim}(X)$, thus we can use (1.13).

\section{Lemma 1.58:}

Assume $\mathcal{A}$ is a terminal order on a smooth projective surface $X$. Let $M$ and $N$ be two coherent $\mathcal{A}$-modules, then there is the following form of Serre duality:

$$
\operatorname{Ext}_{\mathcal{A}}^{i}(M, N) \cong \operatorname{Ext}_{\mathcal{A}}^{2-i}\left(N, \omega_{\mathcal{A}} \otimes_{\mathcal{A}} M\right)^{\prime} .
$$

Here $(-)^{\prime}$ is the $k$-dual.

\section{Proof:}

If $M$ and $N$ are coherent locally projective $\mathcal{A}$-modules, then by the local-to-global spectral sequence:

$$
\begin{aligned}
\operatorname{Ext}_{\mathcal{A}}^{i}(M, N) & =H^{i}\left(X, \mathcal{H o m}_{\mathcal{A}}(M, N)\right) \\
\operatorname{Ext}_{\mathcal{A}}^{2-i}\left(N, \omega_{\mathcal{A}} \otimes_{\mathcal{A}} M\right) & =H^{2-i}\left(X, \mathcal{H o m}_{\mathcal{A}}\left(N, \omega_{\mathcal{A}} \otimes_{\mathcal{A}} M\right)\right) .
\end{aligned}
$$

Letting $M^{\vee}:=\mathcal{H o m}_{\mathcal{A}}(M, \mathcal{A})$, we have $\mathcal{H o m}_{\mathcal{A}}(M, N)=M^{\vee} \otimes_{\mathcal{A}} N$ since $M$ is a coherent locally projective $\mathcal{A}$-module and $\mathcal{A}$ is an $\mathcal{A}$-bimodule. Now we use the tensor-hom-adjunction to see that

$$
\begin{aligned}
M^{*}=\mathcal{H o m}_{\mathcal{O}_{X}}\left(M, \mathcal{O}_{X}\right) & =\mathcal{H o m}_{\mathcal{O}_{X}}\left(\mathcal{A} \otimes_{\mathcal{A}} M, \mathcal{O}_{X}\right) \\
& =\mathcal{H o m}_{\mathcal{A}}\left(M, \mathcal{H o m} \text { O}_{X}\left(\mathcal{A}, \mathcal{O}_{X}\right)\right) \\
& =\mathcal{H o m}_{\mathcal{A}}\left(M, \mathcal{A}^{*}\right) .
\end{aligned}
$$


Using this and the adjunction again we get

$$
\begin{aligned}
\mathcal{H o m}_{\mathcal{O}_{X}}\left(M^{\vee} \otimes_{\mathcal{A}} N, \mathcal{O}_{X}\right) & =\mathcal{H o m}_{\mathcal{A}}\left(N, \mathcal{H o m} \mathrm{O}_{X}\left(M^{\vee}, \mathcal{O}_{X}\right)\right) \\
& =\mathcal{H o m}_{\mathcal{A}}\left(N,\left(M^{\vee}\right)^{*}\right) \\
& =\mathcal{H o m}_{\mathcal{A}}\left(N, \mathcal{H} m_{\mathcal{A}}\left(M^{\vee}, \mathcal{A}^{*}\right)\right) \\
& =\mathcal{H o m}_{\mathcal{A}}\left(N, \mathcal{A}^{*} \otimes_{\mathcal{A}} M^{\vee \vee}\right) .
\end{aligned}
$$

As $M$ is a coherent locally projective $\mathcal{A}$-module, we have $M^{\vee \vee} \cong M$. All together we get

$$
\left(\mathcal{H o m}_{\mathcal{A}}(M, N)\right)^{*}=\mathcal{H o m}_{\mathcal{A}}\left(N, \mathcal{A}^{*} \otimes_{\mathcal{A}} M\right) .
$$

But this implies

$$
\left(\mathcal{H o m}_{\mathcal{A}}(M, N)\right)^{*} \otimes_{\mathcal{O}_{X}} \omega_{X}=\mathcal{H o m}_{\mathcal{A}}\left(N, \mathcal{A}^{*} \otimes_{\mathcal{A}} M\right) \otimes_{\mathcal{O}_{X}} \omega_{X}=\mathcal{H o m}_{\mathcal{A}}\left(N, \omega_{\mathcal{A}} \otimes_{\mathcal{A}} M\right) .
$$

Usual Serre duality on $X$ shows that we have an isomorphism:

$$
H^{i}\left(X, \mathcal{H o m}_{\mathcal{A}}(M, N)\right) \cong H^{2-i}\left(X, \mathcal{H o m}_{\mathcal{A}}\left(N, \omega_{\mathcal{A}} \otimes_{\mathcal{A}} M\right)\right)^{\prime} .
$$

This implies that there is an isomorphism

$$
\operatorname{Ext}_{\mathcal{A}}^{i}(M, N) \cong \operatorname{Ext}_{\mathcal{A}}^{2-i}\left(N, \omega_{\mathcal{A}} \otimes_{\mathcal{A}} M\right)^{\prime}
$$

in this case.

If $M$ and $N$ are not locally projective, then we can choose finite locally projective resolutions since $\mathcal{A}$ has global dimension two and reason like in the proof of [HS05, Proposition 3.5].

\section{Corollary 1.59:}

Assume $M$ is a torsion-free $\mathcal{A}$-module of rank one, then $\operatorname{Hom}_{\mathcal{A}}\left(M, M^{* *}\right)=k$.

\section{Proof:}

We look at the exact sequence

$$
0 \longrightarrow M \longrightarrow M^{* *} \longrightarrow T \longrightarrow 0
$$

and apply $\operatorname{Hom}_{\mathcal{A}}\left(-, M^{* *}\right)$.

Since $T$ is an Artinian sheaf and $M^{* *}$ is torsion-free we have $\operatorname{Hom}_{\mathcal{A}}\left(T, M^{* *}\right)=0$.

Using Serre duality shows that

$$
\operatorname{Ext}_{\mathcal{A}}^{1}\left(T, M^{* *}\right)=\operatorname{Ext}_{\mathcal{A}}^{1}\left(M^{* *}, \omega_{\mathcal{A}} \otimes_{\mathcal{A}} T\right)^{\prime} .
$$

By the local-to-global spectral sequence we get

$$
\operatorname{Ext}_{\mathcal{A}}^{1}\left(M^{* *}, \omega_{\mathcal{A}} \otimes_{\mathcal{A}} T\right)=H^{1}\left(X, \mathcal{H o m}_{\mathcal{A}}\left(M^{* *}, \omega_{\mathcal{A}} \otimes_{\mathcal{A}} T\right)\right),
$$

since $M^{* *}$ is locally projective. But $T$ is Artinian so the $\mathcal{H}$ om-sheaf has support only in dimension zero, so the cohomology group must vanish. This implies $\operatorname{Ext}_{\mathcal{A}}^{1}\left(T, M^{* *}\right)=0$.

Looking at the long exact sequence shows that

$$
\operatorname{Hom}_{\mathcal{A}}\left(M^{* *}, M^{* *}\right) \cong \operatorname{Hom}_{\mathcal{A}}\left(M, M^{* *}\right) .
$$

But $M^{* *}$ is a locally projective $\mathcal{A}$-module of rank one, so $\operatorname{Hom}_{\mathcal{A}}\left(M^{* *}, M^{* *}\right)=k$. 


\section{Lemma 1.60:}

Assume $\mathcal{A}$ is a maximal order on a smooth projective surface $X$ and $I$ is an injective $\mathcal{A}$-module. Then $I$ is an injective $\mathcal{O}_{X}$-module, in other words the forgetful functor $\operatorname{Mod}(\mathcal{A}) \rightarrow \operatorname{Mod}\left(\mathcal{O}_{X}\right)$ preserves injectives.

\section{Proof:}

We have to show that $\operatorname{Hom}_{\mathcal{O}_{X}}(-, I)$ is an exact functor. But since $I$ is an $\mathcal{A}$-module, we have $I=\mathcal{H o m}_{\mathcal{A}}(\mathcal{A}, I)$. Now using the tensor-hom-adjunction gives

$$
\operatorname{Hom}_{\mathcal{O}_{X}}(-, I)=\operatorname{Hom}_{\mathcal{O}_{X}}\left(-, \mathcal{H o m}_{\mathcal{A}}(\mathcal{A}, I)\right)=\operatorname{Hom}_{\mathcal{A}}\left(-\otimes_{\mathcal{O}_{X}} \mathcal{A}, I\right) .
$$

Since $\mathcal{A}$ is a maximal order, it is locally free, so that $\otimes_{\mathcal{O}_{X}} \mathcal{A}$ is exact. Furthermore $\operatorname{Hom}_{\mathcal{A}}(-, I)$ is exact because $I$ is an injective $\mathcal{A}$-module. So $\operatorname{Hom}_{\mathcal{O}_{X}}(-, I)$ is exact and $I$ is an injective $\mathcal{O}_{X}$-module.

\section{Lemma 1.61:}

Assume $\mathcal{A}$ is a maximal order on a smooth projective surface and let $M$ and $N$ be two coherent $\mathcal{A}$-modules. Then there are isomorphisms

$$
\operatorname{Ext}_{\mathcal{A}}^{i}\left(\mathcal{A} \otimes_{\mathcal{O}_{X}} M, N\right) \cong \operatorname{Ext}_{\mathcal{O}_{X}}^{i}(M, N)
$$

for all $i \geq 0$.

\section{Proof:}

The functors $\left\{\operatorname{Ext}_{\mathcal{A}}^{i}\left(\mathcal{A} \otimes_{\mathcal{O}_{X}} M,-\right)\right\}_{i \geq 0}$ are a cohomological $\delta$-functor between $\operatorname{Mod}(\mathcal{A})$ and $A b$. They vanish on injectives in $\operatorname{Mod}(\mathcal{A})$. Similarly the functors $\left\{\operatorname{Ext}_{\mathcal{O}_{X}}^{i}(M,-)\right\}_{i \geq 0}$ are a $\delta$-functor, because the forgetful functor $\operatorname{Mod}(\mathcal{A}) \rightarrow \operatorname{Mod}\left(\mathcal{O}_{X}\right)$ is exact (we should have written $\iota(M)$ and $\iota(-)$ here, where $\iota: \operatorname{Mod}(\mathcal{A}) \rightarrow \operatorname{Mod}\left(\mathcal{O}_{X}\right)$ is the forgetful functor. Since no confusion can arise we will omit this notation). These functors also vanish on injectives in $\operatorname{Mod}(\mathcal{A})$, since by $(1.60)$ the forgeful functor $\operatorname{Mod}(\mathcal{A}) \rightarrow \operatorname{Mod}\left(\mathcal{O}_{X}\right)$ maps injectives to injectives. This implies that both $\delta$-functors are universal.

Using the tensor-hom-adjunction shows that there is a canonical natural equivalence:

$$
\operatorname{Ext}_{\mathcal{A}}^{0}\left(\mathcal{A} \otimes_{\mathcal{O}_{X}} M,-\right)=\operatorname{Hom}_{\mathcal{A}}\left(\mathcal{A} \otimes_{\mathcal{O}_{X}} M,-\right) \cong \operatorname{Hom}_{\mathcal{O}_{X}}(M,-)=\operatorname{Ext}_{\mathcal{O}_{X}}^{0}(M,-) .
$$

So we have two cohomological universal $\delta$-functors which are naturally isomorphic for $i=0$, so they are also naturally isomorphic for all $i \geq 1$.

\section{Lemma 1.62:}

Assume $\mathcal{A}$ is a terminal order on a smooth projective surface $X$ and let $M$ and $S$ be coherent $\mathcal{A}$-modules. If $M$ is locally projective and $S$ is an Artinian module of finite length, then the map

$$
\operatorname{Ext}_{\mathcal{A}}^{2}(S, M) \rightarrow \operatorname{Ext}_{\mathcal{O}_{X}}^{2}(S, M),
$$

induced from the forgetful functor $\operatorname{Mod}(\mathcal{A}) \rightarrow \operatorname{Mod}(X)$, is injective. 


\section{Proof:}

Since $\mathcal{A}$ is an $\mathcal{O}_{X}$-algebra, the multiplication map $\mathcal{A} \times \mathcal{A} \rightarrow \mathcal{A}$ gives rise to a short exact sequence of $\mathcal{A}$-bimodules:

$$
0 \longrightarrow K \longrightarrow \mathcal{A} \otimes_{\mathcal{O}_{X}} \mathcal{A} \longrightarrow \mathcal{A} \longrightarrow 0 .
$$

Here $K$ denotes $\operatorname{ker}\left(\mathcal{A} \otimes_{X} \mathcal{A} \rightarrow \mathcal{A}\right)$.

Since $\mathcal{A}$ is a free $\mathcal{A}$-module, we have $\mathcal{T}_{\text {or }}^{\mathcal{A}}(\mathcal{A}, S)=0$, so by tensoring over $\mathcal{A}$ with $S$ we get the following short exact sequence:

$$
0 \longrightarrow K \otimes_{\mathcal{A}} S \longrightarrow \mathcal{A} \otimes_{\mathcal{O}_{X}} \mathcal{A} \otimes_{\mathcal{A}} S \longrightarrow \mathcal{A} \otimes_{\mathcal{A}} S \longrightarrow 0 .
$$

Using the natural isomorphism of left $\mathcal{A}$-modules $\mathcal{A} \otimes_{\mathcal{A}} S \cong S$ we get the sequence:

$$
0 \longrightarrow K \otimes_{\mathcal{A}} S \longrightarrow \mathcal{A} \otimes_{\mathcal{O}_{X}} S \longrightarrow S \longrightarrow 0 .
$$

Applying $\operatorname{Hom}_{\mathcal{A}}(-, M)$ gives the long exact sequence, with the following relevant part:

$$
\operatorname{Ext}_{\mathcal{A}}^{1}\left(K \otimes_{\mathcal{A}} S, M\right) \longrightarrow \operatorname{Ext}_{\mathcal{A}}^{2}(S, M) \longrightarrow \operatorname{Ext}_{\mathcal{A}}^{2}\left(\mathcal{A} \otimes_{\mathcal{O}_{X}} S, M\right) \longrightarrow \cdots
$$

Using Serre duality shows

$$
\operatorname{Ext}_{\mathcal{A}}^{1}\left(K \otimes_{\mathcal{A}} S, M\right) \cong \operatorname{Ext}_{\mathcal{A}}^{1}\left(M, \omega_{\mathcal{A}} \otimes_{\mathcal{A}} K \otimes_{\mathcal{A}} S\right)^{\prime} .
$$

Denote $\omega_{\mathcal{A}} \otimes_{\mathcal{A}} K \otimes_{\mathcal{A}} S$ by $N$. Since $S$ is of finite length, so is $N$, this shows that

$$
\operatorname{Ext}_{\mathcal{A}}^{1}(M, N)=\bigoplus_{x \in \operatorname{supp}(N)} \operatorname{Ext}_{\mathcal{A}_{x}}^{1}\left(M_{x}, N_{x}\right) .
$$

Since $M$ is a locally projective $\mathcal{A}$-module, for any $x \in X$ the module $M_{x}$ is a projective $\mathcal{A}_{x^{-}}$ module, which implies $\operatorname{Ext}_{\mathcal{A}_{x}}^{1}\left(M_{x}, N_{x}\right)=0$ for all $x$. This shows that $\operatorname{Ext}_{\mathcal{A}}^{1}\left(K \otimes_{\mathcal{A}} S, M\right)=0$.

So the sequence (2) gives an injection:

$$
0 \longrightarrow \operatorname{Ext}_{\mathcal{A}}^{2}(S, M) \longrightarrow \operatorname{Ext}_{\mathcal{A}}^{2}\left(\mathcal{A} \otimes_{\mathcal{O}_{X}} S, M\right) \longrightarrow \cdots .
$$

But by (1.61) there is an isomorphism $\operatorname{Ext}_{\mathcal{A}}^{2}\left(\mathcal{A} \otimes_{\mathcal{O}_{X}} S, M\right) \cong \operatorname{Ext}_{\mathcal{O}_{X}}^{2}(M, N)$. Composing this isomorphism with the injection (3), shows that there is an injection

$$
\operatorname{Ext}_{\mathcal{A}}^{2}(S, M) \hookrightarrow \operatorname{Ext}_{\mathcal{O}_{X}}^{2}(S, M) .
$$

Looking at the proof of (1.61) this map is nothing but the induced map from the forgetful functor from $\mathcal{A}$-modules to $\mathcal{O}_{X}$-modules.

\subsection{Relative Ext-sheaves for modules over an Azumaya algebra}

In this section we want to define relative $\mathcal{E} x t$-sheaves for modules over an Azumaya algebra $\mathcal{A}$. Then we are going to show that there is a base change theorem for theses sheaves, like in the case for $\mathcal{O}_{X}$-modules. Most of this section is a transfer from the situation of $\mathcal{O}_{X}$-modules, given in [Lan83, Chapter 1], to our situtaion.

For the rest of this section we assume that $f: X \rightarrow Y$ is a flat projective morphism of smooth Noetherian schemes. Furthermore we assume that $\mathcal{A}$ is an Azumaya algebra on $X$ flat over $Y$ as an $\mathcal{O}_{X}$-module. In addition $M$ and $N$ are coherent $\mathcal{A}$-modules flat over $Y$ as $\mathcal{O}_{X}$-modules. If $y \in Y$ is a point and $M$ is module on $X$ then the fiber of $f$ over $y$ is given by $X_{y}=X \times_{Y} \operatorname{Spec}(k(y))$ and $M_{y}$ denotes the induced sheaf, that is the pullback of $M$ from $X$ to the fiber $X_{y}$, this should not be confused with the stalk of a module at $y$. 


\section{Definition 1.63:}

For a flat projective morphism $f: X \rightarrow Y$ of smooth Noetherian schemes with an Azumaya algebra $\mathcal{A}$ on $X$ and two coherent $\mathcal{A}$-modules $M$ and $N$ we define the $i$-th relative $\mathcal{E} x$-sheaf by:

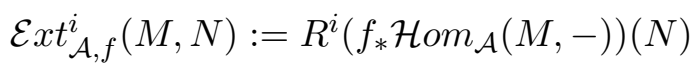

We use that $f$ is projective to define the line bundles $\mathcal{O}_{X}(n)$ on $X$ by fixing a projective embedding $X \hookrightarrow \mathbb{P}_{Y}^{m}$. Consider a morphism $u: Y^{\prime} \rightarrow Y$ of smooth Noetherian schemes and let $X^{\prime}=X \times_{Y} Y^{\prime}$ be the fibred product with the projections $p$ and $q$ to $X$ respectively $Y^{\prime}$.

\section{Lemma 1.64:}

There is an integer $K(N)$, such that $\mathcal{E} x t_{p^{*} \mathcal{A}, q}^{i}\left(p^{*} \mathcal{A}(-n), N \otimes_{Y} P\right)=0$ for all $n \geq K(N), i \geq 1$ and all quasi-coherent $\mathcal{O}_{Y^{\prime}}$-modules $P$.

\section{Proof:}

As $X$ and $Y$ are Noetherian and the question is local we can suppose that $Y=\operatorname{Spec}(R)$ and $Y^{\prime}=\operatorname{Spec}\left(R^{\prime}\right)$ are affine. The fixed projective embedding is then given by $X \hookrightarrow \mathbb{P}_{R}^{m}$.

Since

$$
\mathcal{H o m}_{p^{*} \mathcal{A}}\left(p^{*} \mathcal{A}(-n), N \otimes_{Y} P\right)=\mathcal{H o m}_{p^{*} \mathcal{A}}\left(p^{*} \mathcal{A}, p^{*} N(n) \otimes_{Y^{\prime}} P\right)=p^{*} N(n) \otimes_{Y^{\prime}} P,
$$

we have to show that

$$
R^{i} q_{*}\left(p^{*} N(n) \otimes_{R^{\prime}} P\right)=0
$$

for all $n \geq K(N), i \geq 1$ and $R^{\prime}$-modules $P$.

Consider $N$ as a coherent sheaf on $\mathbb{P}_{R}^{m}$ and replace $X$ by $\mathbb{P}_{R}^{m}$. Then the lemma is true for $i>m$ due to [Har77, Proposition III.8.5].

In the special case $N=\mathcal{A}$ and $P=R^{\prime}$, there is some $n_{0} \in \mathbb{N}$ with $R^{i} q_{*}\left(p^{*} \mathcal{A}(n)\right)=0$ for all $i \geq 1$ and all $n \geq n_{0}$ by the theory of higher direct image sheaves, see [Har77, Theorem III.8.8].

For an arbitrary $R^{\prime}$-module $P$ write

$$
0 \longrightarrow P^{\prime} \longrightarrow R^{\prime(I)} \longrightarrow P \longrightarrow 0 \text {. }
$$

Tensor the sequence with $p^{*} \mathcal{A}(n)$, which is flat over $R^{\prime}$ since $\mathcal{A}$ is flat over R. Now the long exact sequence for $q_{*}$ and the result for $R^{i} q_{*}\left(p^{*} \mathcal{A}(n)\right)$ give isomorphisms for $i \geq 1$ :

$$
R^{i+1} q_{*}\left(p^{*} \mathcal{A}(n) \otimes_{R^{\prime}} P^{\prime}\right) \cong R^{i} q_{*}\left(p^{*} \mathcal{A}(n) \otimes_{R^{\prime}} P\right) .
$$

Since $R^{i+1} q_{*}\left(p^{*} \mathcal{A}(n) \otimes_{R^{\prime}} P^{\prime}\right)=0$ for all $i \geq m$ and all $P^{\prime}$ we can use descending induction to see that $R^{i} q_{*}\left(p^{*} \mathcal{A}(n) \otimes_{R^{\prime}} P\right)=0$ for all $i \geq 1$.

Like in the proof of [HS05, Proposition 3.4] there is an exact sequence of the following form for an arbitrary coherent $\mathcal{A}$-module $N$ :

$$
0 \longrightarrow N^{\prime} \longrightarrow \mathcal{A}(-k)^{r} \longrightarrow N \longrightarrow 0 .
$$

Since $N$ is flat over $Y$ we have $\mathcal{T}_{\text {or }}^{R}\left(N, R^{\prime}\right)=0$ so that the following sequence is exact

$$
0 \longrightarrow p^{*} N^{\prime} \longrightarrow p^{*} \mathcal{A}(-k)^{r} \longrightarrow p^{*} N \longrightarrow 0 .
$$

Now $N$ is flat over $R$ so $p^{*} N$ is flat over $R^{\prime}$, this implies $\mathcal{T}_{0} R_{1}^{\prime}\left(p^{*} N(n), P\right)=0$ so that the following sequence is also exact:

$$
0 \longrightarrow p^{*} N^{\prime}(n) \otimes_{R^{\prime}} P \longrightarrow p^{*} \mathcal{A}(n-k)^{r} \otimes_{R^{\prime}} P \longrightarrow p^{*} N(n) \otimes_{R^{\prime}} P \longrightarrow 0 .
$$

Now the lemma follows by using descending induction one more time. 


\section{Remark 1.65:}

Using this lemma we can make the following convention: whenever we have two coherent $\mathcal{A}$ modules $M$ and $N$, we always choose a locally projective resolution $M_{\bullet} \rightarrow M$ for $M$, where the $M_{i}$ are of the form $\mathcal{A}\left(-n_{i}\right)^{l_{i}}$ with $n_{i} \geq \max \{K(N), G(M)\}$. Here $G(M)$ is the smallest integer with the property that for all $n \geq G(M)$ the coherent sheaf $M(n)$ is generated by a finite number of global sections. Then by the previous lemma we have $\mathcal{E} x t_{p^{*} \mathcal{A}, q}^{i}\left(p^{*} M_{i}, N \otimes_{Y^{\prime}} P\right)=0$ for all $i \geq 1$. This property will be used later. We will denote $\max \{K(N), G(M)\}$ by $l_{M, N}$.

\section{Definition 1.66:}

For two coherent $\mathcal{A}$-modules $M$ and $N$ we define a complex $L \bullet$ of $\mathcal{O}_{Y}$-modules by

$$
L^{i}=f_{*} \mathcal{H o m} m_{\mathcal{A}}\left(M_{i}, N\right)=f_{*} N\left(n_{i}\right)^{l_{i}}
$$

where $M_{\bullet} \rightarrow M$ is a projective resolution of $M$ with properties described in (1.65).

\section{Corollary 1.67:}

$L^{\bullet}$ is a complex of coherent locally free $\mathcal{O}_{Y^{\prime}}$-modules.

\section{Proof:}

Since $f$ is projective and $N$ is coherent we see that the $L^{i}$ are coherent.

It remains to show that each $L^{i}$ is locally free. To do so it is enough that for every $n \geq l_{M, N}$ the sheaf $f_{*} N(n)$ is locally free on $Y$. Since this is a local question we may assume that $Y=\operatorname{Spec}(R)$ is affine and consider the functor $T: \operatorname{Mod}(R) \rightarrow \operatorname{Mod}(R)$ defined by $T(M):=f_{*}\left(N(n) \otimes_{R} M\right)$. By the previous lemma (1.64) $T$ is exact, which by [Har77, Proposition III.12.6] implies that $T(R)$ is a projective $R$-module, hence $f_{*}(N(n))$ is a locally free module on $\operatorname{Spec}(R)$.

\section{Corollary 1.68:}

For every quasi-coherent $\mathcal{O}_{Y^{\prime}}$-module $P$ there is canonical isomorphism

$$
q_{*} \mathcal{H o m}_{p^{*} \mathcal{A}}\left(p^{*} M_{i}, N \otimes_{Y} P\right) \cong L^{i} \otimes_{Y} P
$$

\section{Proof:}

We have a canonical isomorphism

$$
q_{*} \mathcal{H o m} p^{*} \mathcal{A}\left(p^{*} M_{i}, N \otimes_{Y} P\right)=q_{*}\left(p^{*} N\left(n_{i}\right)^{l_{i}} \otimes_{Y^{\prime}} P\right) .
$$

The functor $T: \operatorname{Mod}\left(R^{\prime}\right) \rightarrow \operatorname{Mod}\left(R^{\prime}\right)$ given by $T(P):=q_{*}\left(p^{*} N(n) \otimes_{R^{\prime}} P\right)$ is exact since $p^{*} N$ is flat over $R^{\prime}$ and for $i \geq 1$ all the $R^{i} q_{*}$ vanish by the choice of $n$. As $p^{*} N(n)$ is coherent and flat over $Y^{\prime}$ we can therefore use [Har'77, Proposition III.12.5] to see that there is an isomorphism $T(P) \cong T\left(R^{\prime}\right) \otimes P$. This implies that we have

$$
q_{*}\left(p^{*} N\left(n_{i}\right)^{l_{i}} \otimes_{Y^{\prime}} P\right)=q_{*}\left(p^{*} N\left(n_{i}\right)^{l_{i}}\right) \otimes_{Y^{\prime}} P .
$$

Now by $(1.67) f_{*}\left(N\left(n_{i}\right)\right)$ commutes with base change so we have a canonical isomorphism

$$
q_{*}\left(p^{*} N\left(n_{i}\right)\right)=u^{*}\left(f_{*} N\left(n_{i}\right)\right) .
$$

This implies

$$
q_{*}\left(p^{*} N\left(n_{i}\right)^{l_{i}}\right) \otimes_{Y^{\prime}} P=u^{*} L^{i} \otimes_{Y^{\prime}} P .
$$


And, finally, we see that $u^{*} L^{i} \otimes_{Y^{\prime}} P=L^{i} \otimes_{Y} P$. Putting everything together, there is in fact a canonical isomorphism

$$
q_{*} \mathcal{H o m} p_{p^{*} \mathcal{A}}\left(p^{*} M_{i}, N \otimes_{Y} P\right) \cong L^{i} \otimes_{Y} P
$$

\section{Corollary 1.69:}

For every quasi-coherent $\mathcal{O}_{Y^{\prime}}$-module $P$ and every $i \geq 0$ there is a canonical isomorphism:

$$
\mathcal{E} x t_{p^{*} \mathcal{A}, q}^{i}\left(p^{*} M, N \otimes_{Y} P\right) \cong \mathcal{H}^{i}\left(L^{\bullet} \otimes_{Y} P\right) .
$$

\section{Proof:}

The elements of a locally projective resolution $M_{\bullet}$ of $M$ are flat over $Y$. To see this we note that locally projective over $\mathcal{A}$ implies locally free over $\mathcal{O}_{X}$, because $\mathcal{A}$ is Azumaya. Since each $M_{i}$ is coherent and $X$ is Noetherian this is equivalent to each $M_{i}$ being a flat $\mathcal{O}_{X}$-module. But $X$ is flat over $Y$ so by transitivity of flatness, each $M_{i}$ is flat over $Y$. Using this we will first show that $p^{*} M_{\bullet}$ is a resolution of $p^{*} M$. (We will do this in the case of a resolution of length two, the general case works analogously.)

Assume $M$ has a resolution of the form

$$
0 \longrightarrow M_{2} \longrightarrow M_{1} \stackrel{g}{\longrightarrow} M_{0} \stackrel{f}{\longrightarrow} M \longrightarrow 0 .
$$

Then we get a short exact sequence

$$
0 \longrightarrow \operatorname{ker}(f) \longrightarrow M_{0} \stackrel{f}{\longrightarrow} M \longrightarrow 0,
$$

which shows that $\operatorname{ker}(f)$ is flat over $Y$ since $M$ and $M_{0}$ are. In addition we get an exact sequence on $X \times_{Y} Y^{\prime}$ :

$$
0 \longrightarrow p^{*} \operatorname{ker}(f) \longrightarrow p^{*} M_{0} \stackrel{f}{\longrightarrow} p^{*} M \longrightarrow 0,
$$

since $M$ is flat over $Y$. But we have $\operatorname{ker}(f)=\operatorname{im}(g)$, so that $i m(g)$ is also flat over $Y$. Therefore the exact sequence:

$$
0 \longrightarrow M_{2} \longrightarrow M_{1} \stackrel{g}{\longrightarrow} \operatorname{im}(g) \longrightarrow 0
$$

gives rise to the following short exact sequence

$$
0 \longrightarrow p^{*} M_{2} \longrightarrow p^{*} M_{1} \longrightarrow p^{*} i m(g) \longrightarrow 0 \text {. }
$$

Since $p^{*} k e r(f)=p^{*} i m(g)$ we can glue these short exact sequences back together and get a resolution of $p^{*} M$ :

$$
0 \longrightarrow p^{*} M_{2} \longrightarrow p^{*} M_{1} \longrightarrow p^{*} M_{0} \longrightarrow p^{*} M \longrightarrow 0 .
$$

Now we define $I_{-1}:=p^{*} M$ and let $I_{i}$ be the image of the map $p^{*} M_{i+1} \rightarrow p^{*} M_{i}$ for $i \geq 0$. Then we have

$$
0 \longrightarrow I_{i+1} \longrightarrow p^{*} M_{i+1} \longrightarrow I_{i} \longrightarrow 0
$$

for all $i \geq-1$, which gives us a long exact sequence:

$$
\begin{aligned}
\cdots & \mathcal{E} x t_{p^{*} \mathcal{A}, q}^{r}\left(I_{i}, N \otimes_{Y} P\right) \longrightarrow \mathcal{E} x t_{p^{*} \mathcal{A}, q}^{r}\left(p^{*} M_{i+1}, N \otimes_{Y} P\right) \\
& \longrightarrow \mathcal{E} x t_{p^{*} \mathcal{A}, q}^{r}\left(I_{i+1}, N \otimes_{Y} P\right) \longrightarrow \mathcal{E} x t_{p^{*} \mathcal{A}, q}^{r+1}\left(I_{i}, N \otimes_{Y} P\right) \longrightarrow \cdots
\end{aligned}
$$

But due to the choice of the resolution of $M$ we know that $\mathcal{E} x t_{p^{*} \mathcal{A}, q}^{i}\left(p^{*} M_{i+1}, N \otimes_{Y} P\right)=0$ for all $i \geq 1$, so there are isomorphisms: 


$$
\mathcal{E} x t_{p^{*} \mathcal{A}, q}^{r}\left(I_{i+1}, N \otimes_{Y} P\right) \cong \mathcal{E} x t_{p^{*} \mathcal{A}, q}^{r+1}\left(I_{i}, N \otimes_{Y} P\right)
$$

for all $r \geq 1$. The beginning of the long exact sequnce is

$$
\begin{aligned}
0 \longrightarrow \mathcal{H o m}_{p^{*} \mathcal{A}, q}\left(I_{i}, N \otimes_{Y} P\right) \longrightarrow \mathcal{H o m}_{p^{*} \mathcal{A}, q}\left(p^{*} M_{i+1}, N \otimes_{Y} P\right) & \longrightarrow \mathcal{E x t}_{p^{*} \mathcal{A}, q}^{1}\left(I_{i}, N \otimes_{Y} P\right) \longrightarrow 0 .
\end{aligned}
$$

For $r=0$ the result follows from this exact sequence by setting $i=-1$.

$\mathcal{E} x t_{p^{*} \mathcal{A}, q}^{1}\left(p^{*} M, N \otimes_{Y} P\right)$ is the quotient of $\mathcal{H o m}_{p^{*} \mathcal{A}, q}\left(I_{0}, N \otimes_{Y} P\right)$ by the image of the map

$$
\mathcal{H o m}_{p^{*} \mathcal{A}, q}\left(p^{*} M_{0}, N \otimes_{Y} P\right) \rightarrow \mathcal{H o m}_{p^{*} \mathcal{A}, q}\left(I_{0}, N \otimes_{Y} P\right) .
$$

The exact sequence $p^{*} M_{2} \rightarrow p^{*} M_{1} \rightarrow I_{0} \rightarrow 0$ gives us:

$0 \longrightarrow \mathcal{H o m}_{p^{*} \mathcal{A}, q}\left(I_{0}, N \otimes_{Y} P\right) \longrightarrow \mathcal{H o m}_{p^{*} \mathcal{A}, q}\left(p^{*} M_{1}, N \otimes_{Y} P\right) \longrightarrow \mathcal{H o m}_{p^{*} \mathcal{A}, q}\left(p^{*} M_{2}, N \otimes_{Y} P\right)$

showing that $\mathcal{H o m}_{p^{*} \mathcal{A}, q}\left(I_{0}, N \otimes_{Y} P\right)$ is just the kernel of

$$
\mathcal{H o m}_{p^{*} \mathcal{A}, q}\left(p^{*} M_{1}, N \otimes_{Y} P\right) \rightarrow \mathcal{H o m}_{p^{*} \mathcal{A}, q}\left(p^{*} M_{2}, N \otimes_{Y} P\right) .
$$

On the other hand since $\mathcal{H o m}_{p^{*} \mathcal{A}, q}\left(I_{0}, N \otimes_{Y} P\right) \rightarrow \mathcal{H o m}_{p^{*} \mathcal{A}, q}\left(p^{*} M_{1}, N \otimes_{Y} P\right)$ is injective, the images of

$$
\mathcal{H o m}_{p^{*} \mathcal{A}, q}\left(p^{*} M_{0}, N \otimes_{Y} P\right) \rightarrow \mathcal{H o m}_{p^{*} \mathcal{A}, q}\left(I_{0}, N \otimes_{Y} P\right)
$$

and

$$
\mathcal{H o m}_{p^{*} \mathcal{A}, q}\left(p^{*} M_{0}, N \otimes_{Y} P\right) \rightarrow \mathcal{H o m}_{p^{*} \mathcal{A}, q}\left(p^{*} M_{1}, N \otimes_{Y} P\right)
$$

are isomorphic. This shows that $\mathcal{E} x t_{p^{*} \mathcal{A}, q}^{1}\left(p^{*} M, N \otimes_{Y} P\right)$ is the cohomology of the complex $\mathcal{H o m}_{p^{*} \mathcal{A}, q}\left(p^{*} M_{0}, N \otimes_{Y} P\right) \longrightarrow \mathcal{H o m}_{p^{*} \mathcal{A}, q}\left(p^{*} M_{1}, N \otimes_{Y} P\right) \longrightarrow \mathcal{H o m}_{p^{*} \mathcal{A}, q}\left(p^{*} M_{2}, N \otimes_{Y} P\right)$, which by the previous lemma is the same as the cohomology of the complex

$$
L_{0} \otimes_{Y} P \longrightarrow L_{1} \otimes_{Y} P \longrightarrow L_{2} \otimes_{Y} P .
$$

The same argument works if we replace $p^{*} M$ by $I_{0}$ and

$$
\cdots \rightarrow p^{*} M_{1} \rightarrow p^{*} M_{0} \text { by } \cdots \rightarrow p^{*} M_{2} \rightarrow p^{*} M_{1} .
$$

We can continue and replace $I_{0}$ by $I_{1}$ and so on. Using the isomorphisms

$$
\mathcal{E} x t_{p^{*} \mathcal{A}, q}^{r}\left(I_{i+1}, N \otimes_{Y} P\right) \cong \mathcal{E} x t_{p^{*} \mathcal{A}, q}^{r+1}\left(I_{i}, N \otimes_{Y} P\right) \text { for } r \geq 1,
$$

we reduce by induction to the cases $r=0,1$ which have just been proven.

\section{Definition 1.70:}

For any quasi-coherent $\mathcal{O}_{Y}$-module $P$ we define

$$
\mathcal{T}^{i}(P):=\mathcal{H}^{i}\left(L^{\bullet} \otimes_{Y} P\right) .
$$


Lemma 1.71 ([Har77, Proposition III.12.5]):

There is a canonical homomorphism

$$
\mathcal{T}^{i}\left(\mathcal{O}_{Y}\right) \otimes_{Y} P \rightarrow \mathcal{T}^{i}(P)
$$

\section{Example 1.72:}

The homomorphism from the previous lemma yields for every $u: Y^{\prime} \rightarrow Y$ the base change homomorphism

$$
\tau^{i}(u): u^{*} \mathcal{E} x t_{\mathcal{A}, f}^{i}(M, N) \rightarrow \mathcal{E} x t_{p^{*} \mathcal{A}, q}^{i}\left(p^{*} M, p^{*} N\right) .
$$

\section{Lemma 1.73:}

For every flat morphism $u: Y^{\prime} \rightarrow Y$ of smooth Noetherian schemes and every $i \geq 0$ the base change homomorphism $\tau^{i}(u)$ is an isomorphism.

\section{Proof:}

Take the locally projective resolution $M_{\bullet} \rightarrow M$ as always. Then $p^{*} M_{\bullet} \rightarrow p^{*} M$ is a resolution of $p^{*} M$ on $X \times_{Y} Y^{\prime}$ since $u$ is flat. We know that

$$
\mathcal{H}^{i}\left(Q^{\bullet}\right)=\mathcal{E} x t_{p^{*} \mathcal{A}, q}^{i}\left(p^{*} M, p^{*} N\right)
$$

with $Q^{i}=q_{*} \mathcal{H} o m_{p^{*} \mathcal{A}}\left(p^{*} M_{i}, p^{*} N\right)$. But then

$$
\begin{aligned}
Q^{i} & =q_{*} \mathcal{H}_{\text {om }^{*} \mathcal{A}}\left(p^{*} \mathcal{A}\left(-n_{i}\right)^{l_{i}}, p^{*} N\right) \\
& =q_{*} \mathcal{H o m}_{p^{*} \mathcal{A}}\left(p^{*} \mathcal{A}, p^{*} N\left(n_{i}\right)^{l_{i}}\right) \\
& =q_{*}\left(p^{*} N\left(n_{i}\right)^{l_{i}}\right) .
\end{aligned}
$$

Now as $u: Y^{\prime} \rightarrow Y$ is flat, $f$ is separated and of finite type (because $f$ is projective) and $N\left(n_{i}\right)$ is coherent, we can use the usual base change formula for flat morphisms. So we have $q_{*}\left(p^{*} N\left(n_{i}\right)\right) \cong u^{*}\left(f_{*} N\left(n_{i}\right)\right)$, see [Har77, Proposition III.9.3]. This gives us:

$$
Q^{i} \cong u^{*} f_{*} N\left(n_{i}\right)^{l_{i}}
$$

Using the $\mathcal{A}$-module structure of $N$ this can be written as $Q^{i} \cong u^{*} f_{*} \mathcal{H} m_{\mathcal{A}}\left(M_{i}, N\right)$. But the cohomology of the complex $L^{\bullet}=f_{*} \mathcal{H} \operatorname{Hom}_{\mathcal{A}}\left(M_{\bullet}, N\right)$ is by definition just $\mathcal{H}^{i}\left(L^{\bullet}\right)=\mathcal{E} x t_{\mathcal{A}, f}^{i}(M, N)$. Since $u$ is flat, $u^{*}$ is exact and commutes with cohomology. The cohomology of the complex $u^{*} L^{\bullet}=u^{*} f_{*} \mathcal{H o m}_{\mathcal{A}}\left(M_{\bullet}, N\right)$ is therefore $u^{*} \mathcal{E} x t_{\mathcal{A}, f}^{i}(M, N)$. This gives us an isomorphism

$$
u^{*} \mathcal{E} x t_{\mathcal{A}, f}^{i}(M, N)=\mathcal{H}^{i}\left(u^{*} L^{\bullet}\right) \cong \mathcal{H}^{i}\left(Q^{\bullet}\right)=\mathcal{E} x t_{p^{*} \mathcal{A}, q}^{i}\left(p^{*} M, p^{*} N\right)
$$

\section{Theorem 1.74:}

Let $y \in Y$ be a point and assume that the base change homomorphism

$$
\tau^{i}(y): \mathcal{E} x t_{\mathcal{A}, f}^{i}(M, N) \otimes k(y) \rightarrow \operatorname{Ext}_{\mathcal{A}_{y}}^{i}\left(M_{y}, N_{y}\right)
$$

is surjective. Then

- there is a neighbourhood $U$ of $y$ such that $\tau^{i}\left(y^{\prime}\right)$ is an isomorphism for all $y^{\prime} \in U$; 
- $\tau^{i-1}(y)$ is surjective if and only if $\mathcal{E} x t_{\mathcal{A}, f}^{i}(M, N)$ is locally free in a neighbourhood of $y$.

This can be proved like in [Lan83, Theorem 1.4], by restricting to an open affine set in $Y$.

\section{Lemma 1.75:}

Assume $X$ is a smooth projective surface and let $M$ and $N$ be two coherent locally free $\mathcal{O}_{X^{-}}$ modules. If we have $M \subset N$, then $M \cong N$ or $\operatorname{codim}(\operatorname{supp}(N / M)) \leq 1$.

\section{Proof:}

Assume $M \nsubseteq N$ and $\operatorname{codim}(\operatorname{supp}(N / M))=2$. Then the exact sequence

$$
0 \longrightarrow N \longrightarrow N \longrightarrow N / M \longrightarrow 0
$$

shows that $M$ and $N$ are isomorphic outside a subset $U \subset X$ of codimension two. That is $M \hookrightarrow N$ is an isomorphism on $X \backslash U$. But $M$ and $N$ are locally free, so they are especially reflexive. This implies that we can extend the isomorphism uniquely to an isomorphism to the whole of $X$. But this means $N / M=0$, respectively $M \cong N$. So the assumption was wrong and we must have $\operatorname{codim}(\operatorname{supp}(N / M)) \leq 1$.

\section{Lemma 1.76:}

Assume $X$ is a smooth projective surface. Let $N$ be a coherent torsion-free $\mathcal{O}_{X}$-module and let $M$ be a locally free $\mathcal{O}_{X}$-module. If there is a surjection

$$
M \stackrel{\phi}{\longrightarrow} N \longrightarrow 0
$$

then $\operatorname{ker}(\phi)$ is a locally free $\mathcal{O}_{X}$-module.

\section{Proof:}

Writing $K:=\operatorname{ker}(\phi)$, we get the exact sequence

$$
0 \longrightarrow K \longrightarrow M \stackrel{\phi}{\longrightarrow} N \longrightarrow 0 \text {. }
$$

Since $N$ is torsion-free we have $p d_{\mathcal{O}_{X}}(N) \leq 1$, see [HL10, Chapter 1.1]. As $M$ is locally free we moreover have

$$
p d_{\mathcal{O}_{X}}(K)=\max \left\{0, p d_{\mathcal{O}_{X}}(N)-1\right\} .
$$

which shows that $p d_{\mathcal{O}_{X}}(K)=0$. Because $K$ is coherent this implies that it is in fact a locally free $\mathcal{O}_{X}$-module.

\section{Lemma 1.77:}

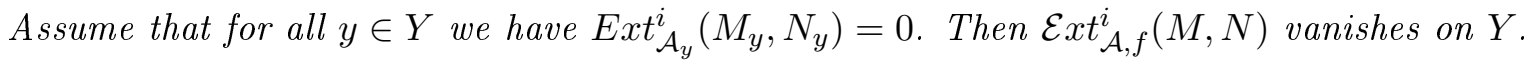

\section{Proof:}

Since $\operatorname{Ext}_{\mathcal{A}_{y}}^{i}\left(M_{y}, N_{y}\right)=0$ the base change theorem gives isomorphisms

$$
\mathcal{E}_{x} t_{\mathcal{A}, f}^{i}(M, N) \otimes k(y) \cong \operatorname{Ext}_{\mathcal{A}_{y}}^{i}\left(M_{y}, N_{y}\right)=0
$$

for all $y \in Y$. But this implies $\mathcal{E} x t_{\mathcal{A}, f}^{i}(M, N)=0$ by the Nakayama lemma. 


\section{Lemma 1.78:}

Assume $Y$ is a smooth projective surface and fix $y_{0} \in Y$. If $\operatorname{Ext}_{\mathcal{A}_{y}}^{i}\left(M_{y}, N_{y}\right)=0$ for all $i \geq 3$ and all $y \in Y$ and furthermore

$$
\begin{gathered}
\operatorname{Hom}_{\mathcal{A}_{y}}\left(M_{y}, N_{y}\right)= \begin{cases}0 & \text { if } y \neq y_{0} \\
\neq 0 & \text { if } y=y_{0}\end{cases} \\
\operatorname{Ext}_{\mathcal{A}_{y}}^{1}\left(M_{y}, N_{y}\right)= \begin{cases}0 & \text { if } y \neq y_{0} \\
\neq 0 & \text { if } y=y_{0}\end{cases} \\
\operatorname{Ext}_{\mathcal{A}_{y}}^{2}\left(M_{y}, N_{y}\right)= \begin{cases}0 & \text { if } y \neq y_{0} \\
k^{s} & \text { if } y=y_{0}\end{cases}
\end{gathered}
$$

Then we have $\mathcal{E} t_{\mathcal{A}, f}^{i}(M, N)=0$ for $i=0,1, i \geq 3$ and $\mathcal{E} x t_{\mathcal{A}, f}^{2}(M, N) \otimes k\left(y_{0}\right) \cong k\left(y_{0}\right)^{s}$.

\section{Proof:}

The statement about $\mathcal{E} x t_{\mathcal{A}, f}^{i}(M, N)$ is clear for $i \geq 3$ by the previous lemma.

There is a complex of coherent locally free sheaves $L^{\bullet}$ on $Y$, whose cohomology is $\mathcal{E x t}_{\mathcal{A}, f}^{\bullet}(M, N)$, such that the cohomology of the complex $L^{\bullet} \otimes k(y)$ is just $\operatorname{Ext}_{\mathcal{A}_{y}}\left(M_{y}, N_{y}\right)$, see the proof of the base change theorem.

Since $\mathcal{E} x t_{\mathcal{A}, f}^{i}(M, N)=0$ for $i \geq 3$ the complex $L^{\bullet}$ is exact at $L^{i}$ for $i \geq 3$.

Now the kernel of the $i$-th differential is the kernel of a surjection from the locally free sheaf $L^{i}$ to the image of the $i$-th differential, which is torsion-free as a subsheaf of $L^{i+1}$. By (1.76) this kernel is locally free.

Replacing $L^{2}$ by $\operatorname{ker}\left(d_{2}\right)$ and using the exactness for $i \geq 3$ we may assume that the complex of locally free sheaves on $Y$ is of the form:

$$
L^{0} \longrightarrow L^{1} \longrightarrow L^{2} .
$$

By assumption $\mathcal{H o m}_{\mathcal{A}, f}(M, N)=\operatorname{ker}\left(d_{0}\right)$ is a sheaf which is concentrated just at $y_{0}$. So it is a torsion sheaf, but also a subsheaf of the locally free sheaf $L^{0}$, so it must be zero.

We see that $\operatorname{ker}\left(d_{1}\right)$ is locally free and contains $L^{0}$ and equals it outside of $y_{0}$ by the assumptions. But then by (1.75) these two locally free sheaves must be equal everywhere, so

$$
\mathcal{E} t_{\mathcal{A}, f}^{1}(M, N)=\operatorname{ker}\left(d_{1}\right) / L^{0}=0 .
$$

For the last statement we use the sequence

$$
0 \longrightarrow i m\left(d_{1}\right) \longrightarrow L^{2} \longrightarrow \mathcal{E} x t_{\mathcal{A}, f}^{2}(M, N) \longrightarrow 0
$$

and tensor it with $k\left(y_{0}\right)$. This shows that

$$
\mathcal{H}^{2}\left(L^{\bullet}\right) \otimes k\left(y_{0}\right)=\left(L^{2} \otimes k\left(y_{0}\right)\right) /\left(i m\left(d_{1}\right) \otimes k\left(y_{0}\right)\right)=\mathcal{H}^{2}\left(L \bullet \otimes k\left(y_{0}\right)\right)=\operatorname{Ext}_{\mathcal{A}_{y}}^{2}\left(M_{y}, N_{y}\right)=k\left(y_{0}\right)^{s} .
$$




\subsection{Chern class computations}

In this chapter we collect some formulas for the Chern classes of the bundles we are interested in. We see these classes as elements in the cohomology with rational coefficients. For a torsion-free module $M$ of rank $r$ on a smooth projective surface $X$ we define the associated determinant line bundle by $\operatorname{det}(M):=(\stackrel{r}{\bigwedge} M)^{* *}$.

\section{Lemma 1.79:}

Assume $X$ is a smooth projective surface and let $M$ and $N$ be coherent $\mathcal{O}_{X}$-modules of rank $r$ respectively s. If one of these modules is locally free, then the Chern classes of the tensor product are given by:

- $c_{1}\left(M \otimes_{\mathcal{O}_{X}} N\right)=s c_{1}(M)+r c_{1}(N)$;

- $c_{2}\left(M \otimes_{\mathcal{O}_{X}} N\right)=s c_{2}(M)+\left(\begin{array}{c}s \\ 2\end{array}\right) c_{1}(M)^{2}+(r s-1) c_{1}(M) c_{1}(N)+\left(\begin{array}{l}r \\ 2\end{array}\right) c_{1}(N)^{2}+r c_{2}(N)$.

\section{Lemma 1.80:}

Let $X$ be a smooth and projective surface and let $\mathcal{T}$ be a torsion sheaf on $X$, sitting in an exact sequence

$$
0 \longrightarrow M \stackrel{\phi}{\longrightarrow} N \longrightarrow \mathcal{T} \longrightarrow 0
$$

where $M$ and $N$ are torsion-free coherent $\mathcal{O}_{X}$-modules of the same rank. Then $c_{1}(\mathcal{T})$ is effective and one has:

$$
c_{1}(\mathcal{T})=\sum_{\operatorname{codim}(\xi)=1} l_{\mathcal{O}_{X, \xi}}\left(\mathcal{T}_{\xi}\right) \overline{\{\xi\}}
$$

\section{Proof:}

Since $M$ and $N$ are torsion-free, the injection $M \hookrightarrow N$ induces an injection $\operatorname{det}(M) \hookrightarrow \operatorname{det}(N)$, see for example [Kob87, Proposition V.6.13]. We get an exact sequence

$$
0 \longrightarrow \operatorname{det}(M) \stackrel{\operatorname{det}(\phi)}{\longrightarrow} \operatorname{det}(N) \longrightarrow \mathcal{Q} \longrightarrow 0 .
$$

Tensoring this exact sequence with the line bundle $\operatorname{det}(N)^{-1}$ shows:

1. $\operatorname{det}(M) \otimes \mathcal{O}_{X}(D) \cong \operatorname{det}(N)$

2. $\mathcal{Q} \cong \mathcal{O}_{D} \otimes \operatorname{det}(N)$

for some effective Cartier divisor $D$. This implies $c_{1}\left(\operatorname{det}(M) \otimes \mathcal{O}_{X}(D)\right)=c_{1}(\operatorname{det}(N))$ or, by using $c_{1}(M)=c_{1}(\operatorname{det}(M))$ :

$$
c_{1}(N)-c_{1}(M)=D .
$$

With the exact sequence (4) and the properties of $c_{1}$ we get: $c_{1}(\mathcal{T})=D$.

Now effective Cartier divisors are the same as effective Weil divisors on $X$, and $D$ corresponds to

$$
\sum_{\operatorname{codim}(\xi)=1} l_{\mathcal{O}_{X, \xi}}\left(\left(\mathcal{O}_{D}\right)_{\xi}\right) \overline{\{\xi\}}
$$


Pick a prime divisor $C$ of $D$ with generic point $\xi$, then $R:=\mathcal{O}_{X, \xi}$ is a discrete valuation ring and the exact sequences (4) and (5) give us two exact sequences:

$$
\begin{gathered}
0 \longrightarrow M_{\xi} \stackrel{\phi_{\xi}}{\longrightarrow} N_{\xi} \longrightarrow \mathcal{T}_{\xi} \longrightarrow 0 \\
0 \longrightarrow \operatorname{det}(M)_{\xi} \stackrel{\operatorname{det}(\phi)_{\xi}}{\longrightarrow} \operatorname{det}(N)_{\xi} \longrightarrow \mathcal{Q}_{\xi} \longrightarrow 0 .
\end{gathered}
$$

The first sequence yields $\mathcal{T}_{\xi}=\operatorname{coker}\left(\phi_{\xi}\right)$ while the second one gives $\left(\mathcal{O}_{D}\right)_{\xi}=R / \operatorname{det}(\phi)_{\xi} R$.

Since $M_{\xi}$ and $N_{\xi}$ are torsion-free, they are in fact free and of the same rank over the principal ideal domain $R$, hence $\operatorname{coker}\left(\phi_{\xi}\right)$ is of finite length. Using the structure theorem for modules over a principal ideal domain, one can see that $l_{R}\left(\operatorname{coker}\left(\phi_{\xi}\right)\right)=l_{R}\left(R / \operatorname{det}\left(\phi_{\xi}\right) R\right)$. So

$$
\begin{aligned}
D & =\sum_{\operatorname{codim}(\xi)=1} l_{R}\left(\left(\mathcal{O}_{D}\right)_{\xi}\right) \overline{\{\xi\}} \\
& =\sum_{\operatorname{codim}(\xi)=1} l_{R}\left(R / \operatorname{det}\left(\phi_{\xi}\right) R\right) \overline{\{\xi\}} \\
& =\sum_{\operatorname{codim}(\xi)=1} l_{R}\left(\operatorname{coker}\left(\phi_{\xi}\right)\right) \overline{\{\xi\}} \\
& =\sum_{\operatorname{codim}(\xi)=1} l_{R}\left(\mathcal{T}_{\xi}\right) \overline{\{\xi\}} .
\end{aligned}
$$

\section{Corollary 1.81:}

Assume $\mathcal{A}$ is a maximal order on a smooth projective surface $X$ and let $M$ and $N$ be torsion-free $\mathcal{A}$-modules of rank one. If $\operatorname{Hom}_{\mathcal{A}}(M, N) \neq 0$ then $c_{1}(N)-c_{1}(M)$ is effective.

\section{Proof:}

By (1.47) a nontrivial element in $\operatorname{Hom}_{\mathcal{A}}(M, N)$ gives rise to a short exact sequence:

$$
0 \longrightarrow M \longrightarrow N \longrightarrow \mathcal{T} \longrightarrow 0 \text {. }
$$

Now use (1.80).

\section{Lemma 1.82 ([Fri98, Chapter 2]):}

Assume $X$ is a smooth and projective surface and $p \in X$ is a closed point. If $I_{p}$ denotes the ideal sheaf of $p$ and $k(p)$ is the skyscraper sheaf at $p$, then we have an exact sequence

$$
0 \longrightarrow I_{p} \longrightarrow \mathcal{O}_{X} \longrightarrow k(p) \longrightarrow 0
$$

which shows that

$$
c_{1}\left(I_{p}\right)=c_{1}(k(p))=0 \text { and } c_{2}\left(I_{p}\right)=-c_{2}(k(p))=1 .
$$

\section{Theorem 1.83:}

Assume $\mathcal{A}$ is a maximal order on a smooth projective surface $X$ of rank $r^{2}$, with ramification curves $\left\{C_{i}\right\}$ and ramification indices $\left\{e_{i}\right\}$ for $i=1, \ldots, l$. Then we have: 


$$
c_{1}(\mathcal{A})=-\frac{r^{2}}{2} \sum_{i=1}^{l}\left(1-\frac{1}{e_{i}}\right) C_{i}
$$

\section{Proof:}

The trace map $\operatorname{tr}: \mathcal{A} \times \mathcal{A} \rightarrow \mathcal{O}_{X}$ defines an exact sequence

$$
0 \longrightarrow \mathcal{A} \longrightarrow \mathcal{A}^{*} \longrightarrow \mathcal{Q} \longrightarrow 0,
$$

which shows that $c_{1}(\mathcal{A})=-\frac{1}{2} c_{1}(\mathcal{Q})$. Now we can compute $c_{1}(\mathcal{Q})$ using $(1.80)$.

We note that if $\xi$ is a point of codimension one in $X$ which is not the generic point of a ramification curve, then $\mathcal{A}_{\xi}$ is Azumaya and the trace gives an identification

$$
\left(\mathcal{A}^{*}\right)_{\xi}=\left(\mathcal{A}_{\xi}\right)^{*} \cong \mathcal{A}_{\xi}
$$

so that $\mathcal{Q}_{\xi}=0$ for these points. This is basically due to the fact that $\mathcal{A}_{\xi}$ gets isomorphic to a matrix algebra over the completion of the local ring $\mathcal{O}_{X, \xi}$. A matrix algebra $M_{n}(R)$ is self-dual with respect to the trace and since the trace is compatible with completion, we get the desired isomorphism.

If $\xi$ is the generic point of a ramification curve $C$ then $\mathcal{A}_{\xi}$ is a maximal $R$-order in $\mathcal{A}_{\eta}$, where $R=\mathcal{O}_{X, \xi}$ is a discrete valuation ring with maximal ideal $\mathfrak{m}$. It is known that maximal orders over discrete valuation rings are standard orders, see for example [Art86, Definition 2.13]. As the length of an $R$-module is preserved under an étale extension $R \rightarrow S$ of discrete valuation rings, we may assume, using Morita equivalence, that $\mathcal{A}_{\xi}$ is of the form

$$
\left(\begin{array}{cccc}
R & \cdots & \cdots & R \\
\mathfrak{m} & R & \ddots & \vdots \\
\vdots & \ddots & \ddots & \vdots \\
\mathfrak{m} & \cdots & \mathfrak{m} & R
\end{array}\right)
$$

Here the matrix is an $e \times e$-matrix, where $e$ is the ramification index of $\mathcal{A}$ over $C$. We see that if $r k(\mathcal{A})=r^{2}$ then $e \mid r$ and we define $f:=\frac{r}{e}$.

The trace pairing then identifies $\left(\mathcal{A}_{\xi}\right)^{*}$ with $\left\{x \in \mathcal{A}_{\eta} \mid \operatorname{tr}\left(x \mathcal{A}_{\xi}\right) \subset R\right\}$, which can easily be computed and is given by:

$$
\left(\mathcal{A}_{\xi}\right)^{*}=\left(\begin{array}{cccc}
R & \cdots & \mathfrak{m}^{-1} & \mathfrak{m}^{-1} \\
R & R & \ddots & \mathfrak{m}^{-1} \\
\vdots & \ddots & \ddots & \vdots \\
R & \cdots & \cdots & R
\end{array}\right)
$$

We conclude that $\mathcal{Q}_{\xi}$ is given in this case by:

$$
\mathcal{Q}_{\xi}=\left(\begin{array}{cccc}
0 & \cdots & \mathfrak{m}^{-1} / R & \mathfrak{m}^{-1} / R \\
R / \mathfrak{m} & 0 & \ddots & \mathfrak{m}^{-1} / R \\
\vdots & \ddots & \ddots & \vdots \\
R / \mathfrak{m} & R / \mathfrak{m} & \cdots & 0
\end{array}\right) .
$$

Now $R / \mathfrak{m}$ is a simple $R$-module and since $R$ is a discrete valuation ring, we have $\mathfrak{m}=(\pi)$ for some uniformizing element $\pi \in R$. This implies $\mathfrak{m}^{-1}=\left(\pi^{-1}\right)$ so that $\mathfrak{m}^{-1} / R=\mathfrak{m}^{-1} / \mathfrak{m m}^{-1} \cong R / \mathfrak{m}$, hence $\mathfrak{m}^{-1} / R$ is also simple. So we have: 


$$
l_{R}(R / \mathfrak{m})=l_{R}\left(\mathfrak{m}^{-1} / R\right)=1 .
$$

Counting the entries above and under the diagonal gives

$$
l_{R}\left(\mathcal{Q}_{\xi}\right)=\frac{e(e-1)}{2} l_{R}(R / \mathfrak{m})+\frac{e(e-1)}{2} l_{R}\left(\mathfrak{m}^{-1} / R\right)=e(e-1) .
$$

Using Morita equivalence shows that, if $r k(\mathcal{A})=r^{2}=(e f)^{2}$, we get

$$
l_{R}\left(\mathcal{Q}_{\xi}\right)=f^{2} e(e-1)=f^{2} e^{2}\left(1-\frac{1}{e}\right)=r^{2}\left(1-\frac{1}{e}\right) .
$$

So we finally get $c_{1}(\mathcal{A})=-\frac{r^{2}}{2} \sum_{i=1}^{l}\left(1-\frac{1}{e_{i}}\right) C_{i}$.

\section{Lemma 1.84:}

Assume $\mathcal{A}$ is a terminal order on a smooth projective surface $X$ with ramification curves $\left\{C_{i}\right\}$ and ramification indices $\left\{e_{i}\right\}$ for $i=1, \ldots, l$. If $M$ is a torsion-free $\mathcal{A}$-module of rank one and $\mathcal{A}^{*}$ denotes the dual sheaf of $\mathcal{A}$, then we have:

$$
c_{1}\left(\mathcal{A}^{*} \otimes_{\mathcal{A}} M\right)=c_{1}(M)-2 c_{1}(\mathcal{A}) .
$$

\section{Proof:}

Since $M$ is torsion-free, we have an exact sequence:

$$
0 \longrightarrow M \longrightarrow M^{* *} \longrightarrow \mathcal{Q} \longrightarrow 0
$$

where $M^{* *}$ is the bidual of $M$ and $\operatorname{codim}(\operatorname{supp}(\mathcal{Q}))=2$. Since $\mathcal{A}^{*}$ is locally free, it is a locally projective $\mathcal{A}$-bimodule by (1.13), particularly it is a flat $\mathcal{A}$-module. So tensoring (6) we get:

$$
0 \longrightarrow \mathcal{A}^{*} \otimes_{\mathcal{A}} M \longrightarrow \mathcal{A}^{*} \otimes_{\mathcal{A}} M^{* *} \longrightarrow \mathcal{A}^{*} \otimes_{\mathcal{A}} \mathcal{Q} \longrightarrow 0
$$

with $\operatorname{codim}\left(\operatorname{supp}\left(\mathcal{A}^{*} \otimes_{\mathcal{A}} \mathcal{Q}\right)\right)=2$. We conclude:

$$
c_{1}\left(\mathcal{A}^{*} \otimes_{\mathcal{A}} M\right)=c_{1}\left(\mathcal{A}^{*} \otimes_{\mathcal{A}} M^{* *}\right) .
$$

This implies that it is enough to prove the lemma for locally projective $\mathcal{A}$-modules.

The trace pairing

$$
\operatorname{tr}: \mathcal{A} \times \mathcal{A} \longrightarrow \mathcal{O}_{X}
$$

gives us an embedding $\mathcal{A} \hookrightarrow \mathcal{A}^{*}$ (which is in fact an isomorphism away from the ramification locus), so there is an exact sequence:

$$
0 \longrightarrow \mathcal{A} \longrightarrow \mathcal{A}^{*} \longrightarrow \mathcal{R} \longrightarrow 0
$$

with $\operatorname{supp}(\mathcal{R}) \subset \bigcup_{i=}^{l} C_{i}$. Using the flatness of the locally projective $\mathcal{A}$-module $M$ we get an exact sequence:

$$
0 \longrightarrow M \longrightarrow \mathcal{A}^{*} \otimes_{\mathcal{A}} M \longrightarrow \mathcal{R} \otimes_{\mathcal{A}} M \longrightarrow 0 .
$$

But by (1.80), we see that $c_{1}\left(\mathcal{A}^{*} \otimes_{\mathcal{A}} M\right)-c_{1}(M)$ must be an effective divisor $D$ and that it has the form

$$
D=\sum_{i=1}^{l} l_{\mathcal{O}_{X, \xi_{i}}}\left(\left(\mathcal{R} \otimes_{\mathcal{A}} M\right)_{\xi_{i}}\right)
$$


where $\xi_{i}$ is the generic point of $C_{i}$.

We are now in the following situation: given a discrete valuation ring $R=\mathcal{O}_{X, \xi}$, with field of fractions $K$, a maximal $R$-order $A=\mathcal{A}_{\xi}$ with ramification index $e$ and a projective $A$-module $N=M_{\xi}$. We want to compute $l_{R}\left(\left(A^{*} \otimes_{A} N\right) / N\right)$.

Since $M$ is a torsion-free $\mathcal{A}$-module of rank one, we know that $M_{\eta}=N \otimes_{R} K$ is a simple $A \otimes_{R} K$ module. By (1.16) $N$ is an indecomposable $A$-module. But then by (1.17) all possible modules $N$ are $A$-isomorphic. So it is enough to compute the length for one $A$-module $N$ and we choose $N=A$. We have to find $l_{R}\left(A^{*} / A\right)$, which is $r^{2}\left(1-\frac{1}{e}\right)$ by looking at the proof of (1.83). We get

$$
D=r^{2} \sum_{i=1}^{l}\left(1-\frac{1}{e_{i}}\right) C_{i} .
$$

So by comparison $D=-2 c_{1}(\mathcal{A})$, which proves that $c_{1}\left(\mathcal{A}^{*} \otimes_{\mathcal{A}} M\right)=c_{1}(M)-2 c_{1}(\mathcal{A})$.

\section{Lemma 1.85:}

Assume $X$ and $Y$ are smooth projective surfaces and $\mathcal{A}$ is an Azumaya algebra on $X$. We have the projections $p$ and $q$ from $X \times Y$ to $X$ respectively $Y$. If $M$ is a coherent $\mathcal{A}$-module and $N$ a coherent $p^{*} \mathcal{A}$-modules, then the class

$$
\operatorname{ch}\left(\mathcal{E} x t_{p^{*} \mathcal{A}, q}^{0}\left(p^{*} M, N\right)-\mathcal{E} x t_{p^{*} \mathcal{A}, q}^{1}\left(p^{*} M, N\right)+\mathcal{E} x t_{p^{*} \mathcal{A}, q}^{2}\left(p^{*} M, N\right)\right)
$$

in $H^{*}(Y, \mathbb{Q})$ depends only on the classes of $\operatorname{ch}(M)$ and $\operatorname{ch}(N)$.

\section{Proof:}

Since $\mathcal{A}$ is Azumaya on $X$, we have that $p^{*} \mathcal{A}$ is Azumaya on $X \times Y$ by (A.9). Thus for all $y \in Y$ and $i \geq 3$ we see:

$$
\mathcal{E} x t_{p^{*} \mathcal{A}, q}^{i}\left(p^{*} M, N\right) \otimes k(y) \cong \operatorname{Ext}_{\mathcal{A}}^{i}\left(M, N_{y}\right)=0
$$

due to the base change theorem and $\left(p^{*} M\right)_{y} \cong M$. So $\mathcal{E} x t_{p^{*} \mathcal{A}, q}^{i}\left(p^{*} M, N\right)=0$ for all $i \geq 3$. Therefore we may assume, like in the proof of (1.78) that there is a complex

$$
L^{0} \longrightarrow L^{1} \longrightarrow L^{2}
$$

of locally free sheaves on $Y$ with $\mathcal{H}^{i}\left(L^{\bullet}\right)=\mathcal{E} x t_{p^{*} \mathcal{A}, q}^{i}\left(p^{*} M, N\right)$.

We see that the class in question is

$$
\operatorname{ch}\left(\mathcal{H}^{0}\left(L^{\bullet}\right)-\mathcal{H}^{1}\left(L^{\bullet}\right)+\mathcal{H}^{2}\left(L^{\bullet}\right)\right) .
$$

Using $\mathcal{H}^{i}\left(L^{\bullet}\right)=\operatorname{ker}\left(d_{i}\right) / \operatorname{im}\left(d_{i-1}\right)$ and the exact sequence

$$
0 \longrightarrow \operatorname{ker}\left(d_{i}\right) \longrightarrow L^{i} \longrightarrow i m\left(d_{i}\right) \longrightarrow 0,
$$

we see that, by additivity of $c h$, the class is:

$$
\operatorname{ch}\left(L^{0}-L^{1}+L^{2}\right) .
$$

Now $L^{i}=q_{*} \mathcal{H}_{0} m_{p^{*} \mathcal{A}}\left(p^{*} M_{i}, N\right)$ where $M_{\bullet} \rightarrow M$ is the locally projective resolution of $M$. Since $p$ is flat $p^{*} M_{\bullet} \rightarrow p^{*} M$ is a locally projective resolution of $p^{*}$ M. Now we use additivity of $c h$ again to get the following class: 


$$
\sum_{i=0}^{2}(-1)^{i} \operatorname{ch}\left(q_{*} \mathcal{H}_{o m} m_{p^{*} \mathcal{A}}\left(p^{*} M_{i}, N\right)\right) .
$$

By assumptions $X \times Y$ is projective, so the projection $q$ is proper. We can therefore apply the Grothendieck-Riemann-Roch theorem for $q$. Since the higher direct images of $\mathcal{H o m}_{p^{*} \mathcal{A}}\left(p^{*} M_{i}, N\right)$ under $q_{*}$ vanish by the choice of the resolution, we get:

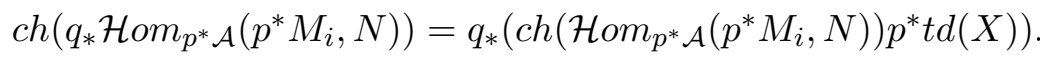

As $p^{*} \mathcal{A}$ is an Azumaya algebra, this class can be simplified using the isomorphism given by $(1.53)$ :

$$
\left(p^{*} \mathcal{A}\right)^{*} \otimes_{\mathcal{O}_{X \times Y}} \mathcal{H o m}_{p^{*} \mathcal{A}}\left(p^{*} M_{i}, N\right)=\mathcal{H o m}_{\mathcal{O}_{X \times Y}}\left(p^{*} M_{i}, N\right) .
$$

But as $p^{*} \mathcal{A}$ is an Azumaya algebra we have $\operatorname{ch}\left(\left(p^{*} \mathcal{A}\right)^{*}\right)=\operatorname{ch}\left(p^{*} \mathcal{A}\right)$. So we get:

$$
\operatorname{ch}\left(\mathcal{H o m}_{p^{*} \mathcal{A}}\left(p^{*} M_{i}, N\right)\right)=\operatorname{ch}\left(\mathcal{H o m}_{\mathcal{O}_{X \times Y}}\left(p^{*} M_{i}, N\right)\right) \operatorname{ch}\left(p^{*} \mathcal{A}\right)^{-1} .
$$

Since $p^{*} M_{i}$ is locally projective over $p^{*} \mathcal{A}$, it is locally free over $\mathcal{O}_{X \times Y}$ by (1.57) so we can further simplify:

$$
\operatorname{ch}\left(\mathcal{H o m}_{\mathcal{O}_{X \times Y}}\left(p^{*} M_{i}, N\right)\right)=\operatorname{ch}\left(\left(p^{*} M_{i}\right)^{*} \otimes N\right)=\operatorname{ch}\left(\left(p^{*} M_{i}\right)^{*}\right) \operatorname{ch}(N) .
$$

Now $p^{*} M_{\bullet} \rightarrow p^{*} M$ is a resolution, so

$$
\sum_{i=0}^{2}(-1)^{i} \operatorname{ch}\left(p^{*} M_{i}\right)=\operatorname{ch}\left(\sum_{i=0}^{2}(-1)^{i} p^{*} M_{i}\right)=\operatorname{ch}\left(p^{*} M\right)
$$

and as the $p^{*} M_{i}$ are locally projective, we get by [HL10, Lemma II.6.1.3] :

$$
\operatorname{ch}\left(\sum_{i=}^{2}(-1)^{i}\left(p^{*} M_{i}\right)^{*}\right)=\operatorname{ch}\left(\sum_{i=0}^{2}(-1)^{i} p^{*} M_{i}\right)^{\vee},
$$

where $\operatorname{ch}(-)^{\vee}$ is the dual class. Putting everything together, we see that the class is given by

$$
q_{*}\left(\operatorname{ch}\left(p^{*} M\right)^{\vee} \operatorname{ch}(N) \operatorname{ch}\left(p^{*} \mathcal{A}\right)^{-1} p^{*} t d(X)\right) .
$$

Using $\operatorname{ch}\left(p^{*} M\right)=p^{*} \operatorname{ch}(M)$ shows, that it does depend only on $\operatorname{ch}(M)$ and $\operatorname{ch}(N)$.

\subsection{Quasi-universal families}

Assume $X$ is a smooth projective surface with a distinguished line bundle $\mathcal{O}_{X}(1)$, so that $X$ is a polarized projective scheme. As the moduli space $M_{\mathcal{A} / X, P}$ is a coarse moduli space, there is no universal family on it. In this section we want to prove that at least a quasi-universal family exists. We will use the notation from [HL10, Chapter 4.6] and adapt the proof given there to our situation. If we have a family of $\mathcal{A}$-modules parametrized by a scheme $S$, then we have the the projections $p: X \times S \rightarrow S$ and $q: X \times S \rightarrow X$.

\section{Definition 1.86:}

A flat family $\mathcal{E}$ of torsion-free $\mathcal{A}$-modules of rank one on $X$ parametrized by $M_{\mathcal{A} / X, P}$ is called quasi-universal, if the following holds: if $\mathcal{F}$ is a family of torsion-free $\mathcal{A}$-modules of rank one with Hilbert polynomial $P$ over $S$ and if $\phi_{\mathcal{F}}: S \rightarrow M_{\mathcal{A} / X, P}, s \mapsto\left[\mathcal{F}_{S}\right]$ is the induced morphism, then there is a locally free $\mathcal{O}_{S}$-module $W$ such that $\mathcal{F} \otimes p^{*} W \cong \phi_{\mathcal{F}, X}^{*} \mathcal{E}$, where $\phi_{\mathcal{F}, X}^{*}:=\left(i d_{X} \times \phi_{\mathcal{F}}\right)^{*}$. 


\section{Remark 1.87:}

By definition for every point $y \in M_{\mathcal{A} / X, P}$ the $\mathcal{A}$-module $\mathcal{E}_{y}$ on $X$ is isomorphic to $M^{\oplus n}$ for a torsion-free $\mathcal{A}$-module $M$ of rank one with Hilbert polynomial $P$, which defines the isomorphism class given by $y \in M_{\mathcal{A} / X, P}$. Here $n$ is the rank of the stalk of $W$ at $y$. If $M_{\mathcal{A} / X ; c_{1}, c_{2}}$ is connected then this number $n$ does not depend on $y$ and is called the similitude of $\mathcal{E}$, see [Muk87, Appendix, Definition A.5].

Let $R$ be the locally closed subscheme in $\operatorname{Quot}\left(\mathcal{A}(-m)^{N}, P\right)$ used in the construction of $M_{\mathcal{A}, P}$ and let $\widetilde{F}$ be the universal quotient on $X \times R$, that is the restriction to $X \times R$ of the universal family on $X \times \operatorname{Quot}\left(\mathcal{A}(-m)^{N}, P\right)$. $\widetilde{F}$ is a $G L(N)$-linearized sheaf on $X \times R$ and since the center $Z$ of $G L(N)$ acts trivially on $R$, see [HS05, Proposition $2.2(\mathrm{v})]$, it has the structure of a $Z$-representaion and decomposes into weight spaces.

\section{Theorem 1.88:}

Quasi-universal families exist.

\section{Proof:}

We first prove that there are $G L(N)$-linearized locally free sheaves of $Z$-weight one on $R$ : if $n$ is sufficiently large $A=p_{*}\left(\widetilde{F} \otimes q^{*} \mathcal{O}_{X}(n)\right)$ is a locally free sheaf on $R$ of rank $P(n)$ and carries a natural $G L(N)$-linearization of $Z$-weight one, the one induced from $\widetilde{F}$.

So let $A$ be any $G L(N)$-linearized locally free sheaf of $Z$ weight one on $R$. Now $Z$ acts trivially on $\mathcal{H o m}\left(p^{*} A, \widetilde{F}\right)$, which therefore carries a $P G L(N)$-linearization and descends to a family $\mathcal{E}$ on $X \times M_{\mathcal{A} / X, P}$ by [HL10, Theorem 4.2.14], because $\pi_{2}: R \longrightarrow M_{\mathcal{A} / X, P}$ is a principal $P G L(N)$ bundle, see [HS05, Theorem 2.4] where it is stated that this morphism is locally trivial in the fppf-topology, but as $P G L(N)$ is smooth it is also locally trivial in the étale topology.

It remains to show that $\mathcal{E}$ is quasi-universal.

Suppose $\mathcal{F}$ is a family of torsion-free $\mathcal{A}$-modules of rank one on $X$ parametrized by a scheme $S$ with Hilbert polynomial $P$. Then $p_{*} \mathcal{F}(m)$ is a locally free $\mathcal{O}_{S}$-module of rank $P(m)=N$. Let

$$
R(\mathcal{F})=\mathbb{I} \operatorname{som}\left(\mathcal{O}_{S}^{N}, p_{*} \mathcal{F}(m)\right) \stackrel{\pi_{1}}{\longrightarrow} S
$$

be the frame bundle. Then, by [HL10, Example 4.2.6], there is a universal $G L(N)$-equivariant trivialization

$$
\mathcal{O}_{R(\mathcal{F})}^{N} \stackrel{\cong}{\longrightarrow} \pi_{1}^{*} p_{*} \mathcal{F}(m)
$$

By the relative version of Serre's theorem we have a surjection

$$
q^{*} \mathcal{O}_{X}(-m) \otimes p^{*} p_{*} \mathcal{F}(m) \longrightarrow \mathcal{F} \longrightarrow 0 .
$$

Now apply $\pi_{1, X}^{*}$ to this surjection, with $\pi_{1, X}=i d_{X} \times \pi_{1}$, and use the universal trivialization to get a quotient

$$
r^{*} \mathcal{O}_{X}(-m)^{N} \otimes s^{*} O_{R(\mathcal{F})} \longrightarrow \pi_{1, X}^{*} \mathcal{F} \longrightarrow 0
$$

on $X \times R(\mathcal{F})$, where $r=q \circ \pi_{1, X}: X \times R(\mathcal{F}) \rightarrow X$ and $s: X \times R(\mathcal{F}) \rightarrow R(\mathcal{F})$ are the projections, and we have $p \circ \pi_{1, X}=\pi_{1} \circ s$. More exactly we have:

$$
\pi_{1, X}^{*} p^{*} p_{*} \mathcal{F}(m)=\left(p \circ \pi_{1, X}\right)^{*} p_{*} \mathcal{F}(m)=\left(\pi_{1} \circ s\right)^{*} p_{*} \mathcal{F}(m)=s^{*}\left(\pi_{1}^{*} p_{*} \mathcal{F}(m)\right)
$$


and the last module can be replaced using the universal quotient.

We can tensor the quotient with $\pi_{1, X}^{*} \mathcal{A}_{S}$ to get a quotient:

$$
r^{*} \mathcal{O}_{X}(-m)^{N} \otimes s^{*} O_{R(\mathcal{F})} \otimes \pi_{1, X}^{*} \mathcal{A}_{S} \longrightarrow \pi_{1, X}^{*} \mathcal{F} \otimes \pi_{1, X}^{*} \mathcal{A}_{S} \longrightarrow 0 .
$$

Since tensor products and pullback commute and $\pi_{1, X}^{*} \mathcal{A}_{S}=\pi_{1, X}^{*} q^{*} \mathcal{A}=r^{*} \mathcal{A}$, we get in fact the following quotient:

$$
r^{*} \mathcal{A}(-m)^{N} \otimes s^{*} O_{R(\mathcal{F})} \longrightarrow \pi_{1, X}^{*}\left(\mathcal{F} \otimes \mathcal{A}_{S}\right) \longrightarrow 0 .
$$

But $\mathcal{F}$ is by definition an $\mathcal{A}_{S}$-module, so we get a surjection

$$
\mathcal{F} \otimes \mathcal{A}_{S} \longrightarrow \mathcal{F} \longrightarrow 0
$$

using the $\mathcal{A}_{S}$-module structure. Applying $\pi_{1, X}^{*}$ to the last equation and combining this with (7), we get a quotient:

$$
r^{*} \mathcal{A}(-m)^{N} \otimes s^{*} O_{R(\mathcal{F})} \longrightarrow \pi_{1, X}^{*} \mathcal{F} \longrightarrow 0
$$

of $\mathcal{A}(-m)^{N}$ on $X \times R(\mathcal{F})$.

As $Q u o t\left(\mathcal{A}(-m)^{N}, P\right)$ represents the $\mathcal{Q}$ uot-functor, this quotient gives rise to a map

$$
\widetilde{\phi}_{\mathcal{F}}: R(\mathcal{F}) \rightarrow \operatorname{Quot}\left(\mathcal{A}(-m)^{N}, P\right) .
$$

Now $G L(N)$ acts on $R(\mathcal{F})$ from the right by composition, so that the frame bundle is in fact a principal $G L(N)$-bundle, see [HL10, Example 4.2.3]. The morphism $\widetilde{\phi}_{\mathcal{F}}$ is $G L(N)$-equivariant and by construction we have $\bar{\phi}_{\mathcal{F}}(R(\mathcal{F})) \subset R$.

Thus we can consider the commutative diagram

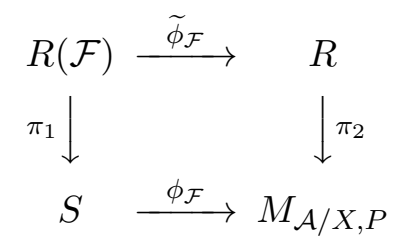

Since $\pi_{2, X}^{*} \mathcal{E} \cong \mathcal{H o m}\left(p^{*} A, \widetilde{F}\right)$ we have:

$$
\pi_{1, X}^{*} \phi_{\mathcal{F}, X}^{*} \mathcal{E} \cong \widetilde{\phi}_{\mathcal{F}, X}^{*} \pi_{2, X}^{*} \mathcal{E} \cong \widetilde{\phi}_{\mathcal{F}, X}^{*} \mathcal{H o m}\left(p^{*} A, \widetilde{F}\right) \cong \mathcal{H o m}\left(\widetilde{\phi}_{\mathcal{F}, X}^{*} p^{*} A, \widetilde{\phi}_{\mathcal{F}, X}^{*} \widetilde{F}\right)
$$

Now by definition: $\widetilde{\phi}_{\mathcal{F}, X}^{*} \widetilde{F} \cong \pi_{1, X}^{*} \mathcal{F}$ and $\widetilde{\phi}_{\mathcal{F}, X}^{*} p^{*} A \cong s^{*} \widetilde{\phi}_{\mathcal{F}}^{*} A$.

Since $\widetilde{\phi}_{\mathcal{F}}^{*} A$ is $G L(N)$-linearized in a natural way and $\pi_{1}: R(\mathcal{F}) \rightarrow S$ is a $G L(N)$-principal bundle, there is a locally free sheaf $B$ on $S$ such that there is an isomorphism $\widetilde{\phi}_{\mathcal{F}}^{*} A \cong \pi_{1}^{*} B$.

Furthermore we have $s^{*} \widetilde{\phi}_{\mathcal{F}}^{*} A \cong \pi_{1, X}^{*} p^{*} B$. So we conclude:

$$
\pi_{1, X}^{*} \phi_{\mathcal{F}, X}^{*} \mathcal{E} \cong \mathcal{H o m}\left(\pi_{1, X}^{*} p^{*} B, \pi_{1, X}^{*} \mathcal{F}\right)=\pi_{1, X}^{*} \mathcal{H o m}\left(p^{*} B, \mathcal{F}\right)
$$

which is equivariant. Using [HL10, 4.2.14] we see that this map descends to an isomorphism:

$$
\phi_{\mathcal{F}, X}^{*} \mathcal{E} \cong \mathcal{H o m}\left(p^{*} B, \mathcal{F}\right) \cong p^{*} B^{\vee} \otimes \mathcal{F} .
$$

So $\mathcal{E}$ is in fact a quasi-universal family. 


\section{Moduli spaces over K3 and abelian surfaces}

If $X$ is a smooth $K 3$ or abelian surface and $E$ a coherent $\mathcal{O}_{X}$-module, then Mukai defined in [Muk87] a class $v(E) \in H^{e v}(X, \mathbb{Q})=\bigoplus_{i=0}^{2} H^{2 i}(X, \mathbb{Q})$, today called Mukai vector of $E$, by

$$
v(E):=\operatorname{ch}(E) \sqrt{\operatorname{td}(X)} .
$$

Here $\operatorname{ch}(E)$ is the Chern Character of $E$ and $t d(X)$ is the Todd class of $X$, both classes belong to $H^{e v}(X, \mathbb{Q})$. For every $u=(a, b, c)$ and $u^{\prime}=\left(a^{\prime}, b^{\prime}, c^{\prime}\right)$ in $H^{e v}(X, \mathbb{Q})$ he defined a symmteric bilinear form $(-,-)$, using the cup product, by:

$$
\left(u, u^{\prime}\right)=b \cup b^{\prime}-a \cup c^{\prime}-a^{\prime} \cup c
$$

He observed that $\left(u, u^{\prime}\right)$ is equal to the $H^{4}(X, \mathbb{Q})$ component of $-u \cdot u^{\prime} \in H^{e v}(X, \mathbb{Q})$, the usual product. Writing $u=\oplus u_{i}$ we can define the dual class $u^{\vee}=\oplus(-1)^{i} u_{i}$. So we can write:

$$
\left(u, u^{\prime}\right)=-\int_{X} u^{\vee} \cdot u^{\prime}
$$

where $\int_{X}$ means taking the $H^{4}(X, \mathbb{Q})$ componenet of the product. For two coherent $\mathcal{O}_{X}$-modules $E$ and $F$ the product $(v(E), v(F))$ equals the $H^{4}$ component of $-\operatorname{ch}(E)^{\vee} \cdot \operatorname{ch}(F) \cdot \operatorname{td}(X)$. Using the Hirzebruch-Riemann-Roch theorem he proved:

\section{Proposition 2.1:}

Assume $E$ and $F$ are coherent $\mathcal{O}_{X}$-modules and put

$$
\chi(E, F)=\sum_{i=0}^{2}(-1)^{i} \operatorname{dim}\left(E x t_{\mathcal{O}_{X}}^{i}(E, F)\right) .
$$

Then we have $\chi(E, F)=-(v(E), v(F))$.

Denote by $M(v)$ the moduli space of stable sheaves on $X$ with Mukai vector $v$, then it is known that $M(v)$ is smooth and that the there is canonical isomorphism

$$
T_{[E]} M(v) \cong \operatorname{Ext}_{\mathcal{O}_{X}}^{1}(E, E)
$$

for the tangent space at a point $[E] \in M(v)$. Since $E$ is stable it is a simple $\mathcal{O}_{X}$-module, so we have $\operatorname{Hom}_{\mathcal{O}_{X}}(E, E)=k$. Using the fact that $\omega_{X} \cong \mathcal{O}_{X}$ and Serre duality gives $\operatorname{Ext}_{\mathcal{O}_{X}}^{2}(E, E) \cong$ $\operatorname{Hom}_{\mathcal{O}_{X}}(E, E)^{\prime}=k$. This shows

$$
\operatorname{dim}\left(\operatorname{Ext}_{\mathcal{O}_{X}}^{1}(E, E)\right)=2+(v(E), v(E)) .
$$

So $M(v)$ has dimension $(v, v)+2$. Using these results Mukai proved the following two theorems:

\section{Theorem 2.2:}

Assume $v$ is a Mukai vector with $(v, v)=-2$, then $M(v)$ is either empty or a reduced point.

\section{Theorem 2.3:}

Assume $v$ is an isotropic Mukai vector, that is $(v, v)=0$. If $M(v)$ contains a connected component $M$ which is compact, then we have: 
1. $M(v)$ is irreducible;

2. every semistable sheaf $K$ with $v(K)=v$ is stable.

In this section we will adapt these definitions to the situation of torsion-free $\mathcal{A}$-modules on a $K 3$ or an abelian surface and obtain similiar results if $\mathcal{A}$ is unramified, that is an Azumaya algebra on $X$.

\subsection{Euler characteristic and Mukai vectors for modules over orders Definition 2.4:}

Assume $\mathcal{A}$ is a terminal order on a smooth projective surface and let $M$ and $N$ be coherent $\mathcal{A}$-modules, then we define the $\mathcal{A}$-Euler characteristic of the pair $(M, N)$ by:

$$
\chi_{\mathcal{A}}(M, N):=\sum_{i=0}^{2}(-1)^{i} \operatorname{dim}_{k}\left(\operatorname{Ext}_{\mathcal{A}}^{i}(M, N)\right)
$$

\section{Lemma 2.5:}

Assume $\mathcal{A}$ is a terminal order on a smooth projective surface $X$. If $0 \rightarrow M^{\prime} \rightarrow M \rightarrow M^{\prime \prime} \rightarrow 0$ is an exact sequence of coherent $\mathcal{A}$-modules, then we have:

$$
\chi_{\mathcal{A}}(M, N)=\chi_{\mathcal{A}}\left(M^{\prime}, N\right)+\chi_{\mathcal{A}}\left(M^{\prime \prime}, N\right)
$$

\section{Proof:}

Since the category $R$-Mod of left $R$-module has enough injectives for any ring $R$, we see that we can adapt the proof of [Har77, Proposition III.2.2] to see that the category $\operatorname{Mod}(\mathcal{A})$ has enough injectives. So we choose an injective resolution $0 \rightarrow N \rightarrow I^{\bullet}$.

Since the $I^{j}$ are injective, the functors $\operatorname{Hom}_{\mathcal{A}}\left(-, I^{j}\right)$ are exact, so we get a short exact sequence of complexes

$$
0 \rightarrow \operatorname{Hom}_{\mathcal{A}}\left(M^{\prime \prime}, I^{\bullet}\right) \rightarrow \operatorname{Hom}_{\mathcal{A}}\left(M, I^{\bullet}\right) \rightarrow \operatorname{Hom}_{\mathcal{A}}\left(M^{\prime}, I^{\bullet}\right) \rightarrow 0 .
$$

Taking the long exact sequence of cohomology groups gives the long exact $\operatorname{Ext}_{\mathcal{A}}$-sequence. By (1.52) we have $\operatorname{Ext}_{\mathcal{A}}^{i}(M, N)=0$ for $i \geq 3$. Using the fact that Euler characteristic in an exact sequence is zero and grouping the Ext according to $M, M^{\prime}$ and $M^{\prime \prime}$ shows

$$
\chi_{\mathcal{A}}\left(M^{\prime}, N\right)-\chi_{\mathcal{A}}(M, N)+\chi_{\mathcal{A}}\left(M^{\prime \prime}, N\right)=0 .
$$

\section{Lemma 2.6:}

Assume $\mathcal{A}$ is a terminal order on a smooth projective surface $X$. Let $M$ be a coherent locally projective $\mathcal{A}$-module and let $N$ be a coherent $\mathcal{A}$-module. Then we have:

$$
\chi_{\mathcal{A}}(M, N)=\chi\left(X, \mathcal{H o m}_{\mathcal{A}}(M, N)\right)=\int_{X} \operatorname{ch}\left(\mathcal{H o m}_{\mathcal{A}}(M, N)\right) \cdot \operatorname{td}(X) .
$$

\section{Proof:}

Since $M$ is locally projective the local-to-global spectral sequence shows that we have

$$
\operatorname{Ext}_{\mathcal{A}}^{i}(M, N)=H^{i}\left(X, \mathcal{H o m}_{\mathcal{A}}(M, N)\right) .
$$


This implies that the $\mathcal{A}$-Euler characteristic for $M$ and $N$ agrees with the usual Euler characteristic for $\mathcal{H o m}_{\mathcal{A}}(M, N)$, meaning $\chi_{\mathcal{A}}(M, N)=\chi\left(X, \mathcal{H o m}_{\mathcal{A}}(M, N)\right)$. Now the last assertion is just the Hirzebruch-Riemann-Roch theorem for $\mathcal{H o m}_{\mathcal{A}}(M, N)$.

\section{Definition 2.7:}

Let $M$ be a coherent $\mathcal{A}$-module, then we define the $\mathcal{A}$-Chern character by:

$$
\operatorname{ch}_{\mathcal{A}}(M):=\operatorname{ch}(M) \cdot{\sqrt{\operatorname{ch}\left(\mathcal{A}^{*}\right)}}^{-1} .
$$

\section{Remark 2.8:}

By $\sqrt{-}$ we mean the positive square root, that is the degree zero component is positive. Since $r k(\mathcal{A})=r^{2}$ with $r \geq 1$ the class $\sqrt{c h(\mathcal{A})}^{-1}$ is well defined and can be found with a power series expansion.

\section{Lemma 2.9:}

Assume $\mathcal{A}$ is a terminal order on a smooth projective surface $X$. If $M$ is a coherent locally projective $\mathcal{A}$-module and $N$ is a coherent $\mathcal{A}$-module, then we have:

$$
\operatorname{ch}\left(\mathcal{H o m}{ }_{\mathcal{A}}(M, N)\right)=\operatorname{ch}_{\mathcal{A}}\left(M^{*}\right) \cdot \operatorname{ch}_{\mathcal{A}}(N) .
$$

\section{Proof:}

By (1.53) we have an isomorphism $\mathcal{A}^{*} \otimes_{\mathcal{O}_{X}} \mathcal{H o m}_{\mathcal{A}}(M, N) \cong \mathcal{H o m}_{\mathcal{O}_{X}}(M, N)$. This implies

$$
\operatorname{ch}\left(\mathcal{A}^{*} \otimes_{\mathcal{O}_{X}} \mathcal{H o m}_{\mathcal{A}}(M, N)\right)=\operatorname{ch}\left(\mathcal{H o m}_{\mathcal{O}_{X}}(M, N)\right) .
$$

Since $\mathcal{A}^{*}$ is a locally free $\mathcal{O}_{X}$-module, the Chern character is multiplicative with respect to the tensor product:

$$
\operatorname{ch}\left(\mathcal{A}^{*} \otimes_{\mathcal{O}_{X}} \mathcal{H o m}{ }_{\mathcal{A}}(M, N)\right)=\operatorname{ch}\left(\mathcal{A}^{*}\right) \operatorname{ch}\left(\mathcal{H o m} m_{\mathcal{A}}(M, N)\right) .
$$

But $M$ is a locally projective $\mathcal{A}$-module, so by $(1.55)$ it is a locally free $\mathcal{O}_{X}$-module, which implies that $\mathcal{H o m}_{\mathcal{O}_{X}}(M, N)=M^{*} \otimes_{\mathcal{O}_{X}} N$. Using again the multiplicativity of $c h$, we get

$$
\operatorname{ch}\left(\mathcal{H o m}_{\mathcal{O}_{X}}(M, N)\right)=\operatorname{ch}\left(M^{*}\right) \operatorname{ch}(N) .
$$

So, finally, we see:

$$
\begin{aligned}
\operatorname{ch}\left(\mathcal{H o m}_{\mathcal{A}}(M, N)\right) & =\operatorname{ch}\left(\mathcal{H o m}_{\mathcal{O}_{X}}(M, N)\right) \operatorname{ch}\left(\mathcal{A}^{*}\right)^{-1} \\
& =\operatorname{ch}\left(M^{*}\right){\sqrt{\operatorname{ch}\left(\mathcal{A}^{*}\right)}}^{-1} \operatorname{ch}(N){\sqrt{\operatorname{ch}\left(\mathcal{A}^{*}\right)}}^{-1} \\
& =\operatorname{ch}_{\mathcal{A}}\left(M^{*}\right) \operatorname{ch}_{\mathcal{A}}(N) .
\end{aligned}
$$

\section{Corollary 2.10:}

Assume $\mathcal{A}$ is a terminal order on a smooth projective surface $X$. If $M$ is a locally projective $\mathcal{A}$-module and $N$ is a coherent $\mathcal{A}$-module, then we have:

$$
\chi_{\mathcal{A}}(M, N)=\int_{X} c h_{\mathcal{A}}\left(M^{*}\right) \cdot c h_{\mathcal{A}}(N) \cdot t d(X) .
$$




\section{Proof:}

This follows from (2.6) and (2.9).

\section{Remark 2.11:}

By choosing a locally projective resolution for a coherent $\mathcal{A}$-module $M$, we see that the previous corollary is in fact correct for all coherent $\mathcal{A}$-modules $M$ and $N$.

\section{Definition 2.12:}

If $\mathcal{A}$ is a terminal order on a smooth projective surface $X$ and $M$ is a coherent $\mathcal{A}$-module we define its $\mathcal{A}$-Mukai vector by:

$$
v_{\mathcal{A}}(M)=\operatorname{ch}_{\mathcal{A}}(M) \cdot \sqrt{t d(X)} .
$$

\section{Example 2.13:}

Assume $X$ is a $K 3$ or an abelian surface. If $\mathcal{A}$ is an Azumaya algebra with $r k(\mathcal{A})=r^{2}$, then $\mathcal{A}^{*} \cong \mathcal{A}$ using the trace map. So $c_{1}(\mathcal{A})=c_{1}\left(\mathcal{A}^{*}\right)=-c_{1}(\mathcal{A})$ implying $c_{1}(\mathcal{A})=0$. Thus we have $\operatorname{ch}(\mathcal{A})=r^{2}-c_{2}(\mathcal{A})$. Using $(\sqrt{1-x})^{-1} \approx 1+\frac{1}{2} x$ shows that

$$
\sqrt{\operatorname{ch}(\mathcal{A})}^{-1}=\frac{1}{r}+\frac{1}{2 r^{3}} c_{2}(\mathcal{A}) \text {. }
$$

Furthermore since $X$ is abelian or $K 3$ we have $K_{X}=0$ so that $t d(X)=1+\chi\left(\mathcal{O}_{X}\right)$ and with $\sqrt{1+x} \approx 1+\frac{1}{2} x$ this gives

$$
\sqrt{t d(X)}=1+\frac{1}{2} \chi\left(\mathcal{O}_{X}\right)
$$

(Here we have to be a little bit careful how to read this: $1 \in H^{0}(X, \mathbb{Q})$ but $\frac{1}{2} \chi\left(\mathcal{O}_{X}\right) \in H^{4}(X, \mathbb{Q})$ !). Now assume $M$ is a coherent $\mathcal{A}$-module with $r k(M)=s$ as an $\mathcal{O}_{X}$-module and Chern classes $c_{1}$ and $c_{2}$, then $\operatorname{ch}(M)=s+c_{1}+\frac{1}{2}\left(c_{1}^{2}-2 c_{2}\right)$.

We compute the $\mathcal{A}$-Mukai vector of $M$ and get:

$$
v_{\mathcal{A}}(M)=\left(\frac{s}{r}, \frac{1}{r} c_{1}, \frac{1}{2 r}\left(c_{1}^{2}-2 c_{2}+\frac{s}{r^{2}} c_{2}(\mathcal{A})\right)+\frac{s}{2 r} \chi\left(\mathcal{O}_{X}\right)\right)
$$

For example if $M$ is a torsion-free $\mathcal{A}$-module of rank one, then $r k(M)=r^{2}$, so we get:

$$
v_{\mathcal{A}}(M)=\left(r, \frac{1}{r} c_{1}, \frac{1}{2 r}\left(c_{1}^{2}-2 c_{2}+c_{2}(\mathcal{A})\right)+\frac{r}{2} \chi\left(\mathcal{O}_{X}\right)\right) .
$$

If $S$ is an Artinian $\mathcal{A}$-module of length $l_{\mathcal{A}}(S)=n$, then $r k(S)=0, c_{1}(S)=0$ and $c_{2}(S)=-n$ so that

$$
v_{\mathcal{A}}(S)=\left(0,0, \frac{n}{r}\right)
$$

The last formula is also valid for a terminal order $\mathcal{A}$ on a smooth projective surface, since $\operatorname{ch}(S)=n$ only lives in $H^{4}(X, \mathbb{Q})$.

\section{Lemma 2.14:}

Assume $\mathcal{A}$ is a terminal order on smooth projective surface $X$. If $M$ and $N$ are coherent $\mathcal{A}$ modules, then we have

$$
\chi_{\mathcal{A}}(M, N)=-\left(v_{\mathcal{A}}(M), v_{\mathcal{A}}(N)\right),
$$


where the bilinear form $(-,-)$ on $H^{e v}(X, \mathbb{Q})=\bigoplus H^{2 i}(X, \mathbb{Q})$ is given by $(v, w)=-\int_{X} v^{\vee} . w$ as described above.

\section{Proof:}

If $\mathcal{A}$ is an Azumaya algebra, then, due to (2.10), the left hand side is given by the $H^{4}$ component of

$$
\operatorname{ch}(M)^{\vee} \cdot \operatorname{ch}(N) \cdot \operatorname{td}(X) \cdot \operatorname{ch}(\mathcal{A})^{-1} .
$$

Using $t d(X) \cdot \operatorname{ch}(\mathcal{A})^{-1}=\frac{1}{r^{2}}+\frac{1}{r^{4}} c_{2}(\mathcal{A})+\frac{1}{r^{2}} \chi\left(\mathcal{O}_{X}\right)$ we see that, if $r k(M)=s$ and $r k(N)=t$, the left hand side is given by:

$$
\frac{s t}{r^{2}} \chi\left(\mathcal{O}_{X}\right)+\frac{s t}{r^{4}} c_{2}(\mathcal{A})+\frac{s}{r^{2}} c h_{2}(N)+\frac{t}{r^{2}} c h_{2}(M)-\frac{1}{r^{2}} c_{1}(N) c_{1}(M) .
$$

Here $c h_{2}=\frac{1}{2}\left(c_{1}^{2}-2 c_{2}\right)$. Now writing the Mukai vectors as

$$
v_{\mathcal{A}}(M)=\left(\frac{s}{r}, \frac{1}{r} c_{1}, \frac{1}{2 r}\left(2 c h_{2}(M)+\frac{s}{r^{2}} c_{2}(\mathcal{A})\right)+\frac{s}{2 r} \chi\left(\mathcal{O}_{X}\right)\right)
$$

shows that the right hand side is given by the same term.

If $\mathcal{A}$ is ramified, then $c_{1}(\mathcal{A}) \neq 0$ and the computations are tedious but show the same result.

\section{Example 2.15:}

Assume $\mathcal{A}$ is an Azumaya algebra. We see that if $v=(a, b, c)$ then $(v, v)=b^{2}-2 a c$. Using the description of $v_{\mathcal{A}}(M)$ from (2.13) for a torsion-free $\mathcal{A}$-module $M$ of rank one with Chern classes $c_{1}$ and $c_{2}$, we get

$$
\left(v_{\mathcal{A}}(M), v_{\mathcal{A}}(M)\right)=\frac{1}{r^{2}} c_{1}^{2}-\left(c_{1}^{2}-2 c_{2}\right)-c_{2}(\mathcal{A})-r^{2} \chi\left(\mathcal{O}_{X}\right) .
$$

The term $\frac{1}{r^{2}} c_{1}^{2}-\left(c_{1}^{2}-2 c_{2}\right)$ can be simplified to $\frac{1}{r^{2}}\left(2 r^{2} c_{2}-\left(r^{2}-1\right) c_{1}^{2}\right)$. So if $\Delta=2 r^{2} c_{2}-\left(r^{2}-1\right) c_{1}^{2}$ denotes the discriminant of $M$ we have

$$
\left(v_{\mathcal{A}}(M), v_{\mathcal{A}}(M)\right)=\frac{1}{r^{2}} \Delta-c_{2}(\mathcal{A})-r^{2} \chi\left(\mathcal{O}_{X}\right)
$$

Since a torsion-free $\mathcal{A}$-module $M$ of rank one is simple (1.49), we have $\operatorname{Hom}_{\mathcal{A}}(M, M)=k$ and using Serre duality shows that $\operatorname{Ext}_{\mathcal{A}}^{2}(M, M)=\operatorname{Hom}_{\mathcal{A}}(M, M)^{\prime}=k$. So we get

$$
\operatorname{dim}\left(\operatorname{Ext}_{\mathcal{A}}^{1}(M, M)\right)=2+\left(v_{\mathcal{A}}(M), v_{\mathcal{A}}(M)\right),
$$

or $\operatorname{dim}\left(\operatorname{Ext}_{\mathcal{A}}^{1}(M, M)\right)=2+\frac{1}{r^{2}} \Delta-c_{2}(\mathcal{A})-r^{2} \chi\left(\mathcal{O}_{X}\right)$. Now if we compare this with the dimension formula for the moduli space $M_{\mathcal{A} / X ; c_{1}, c_{2}}$ given in (3.1), we see that

$$
\operatorname{dim}\left(M_{\mathcal{A} / X ; c_{1}, c_{2}}\right)=2+\left(v_{\mathcal{A}}(M), v_{\mathcal{A}}(M)\right)
$$

which is the same type of formula as in the case of stable sheaves.

\section{Example 2.16:}

Assume $\mathcal{A}$ is a terminal order and $S$ is an Artinian $\mathcal{A}$-module of length $n$. Then we know that $v_{\mathcal{A}}(S)=\left(0,0, \frac{n}{r}\right)$. So $\left(v_{\mathcal{A}}(S), v_{\mathcal{A}}(S)\right)=0$ which implies that $\chi_{\mathcal{A}}(S, S)=0$. 


\subsection{Zero-dimensional moduli spaces}

Using the $\mathcal{A}$-Mukai vector, we will prove a theorem similar to Mukai's result (2.2).

\section{Theorem 2.17:}

Assume $X$ is a $K 3$ or an abelian surface and let $\mathcal{A}$ be an Azumaya algebra on $X$. If $v_{\mathcal{A}}$ is a Mukai vector such that $\left(v_{\mathcal{A}}, v_{\mathcal{A}}\right)=-2$, then either the moduli space is empty or $\operatorname{dim}\left(M_{\mathcal{A} / X ; c_{1}, c_{2}}\right)=0$. If $M_{\mathcal{A} / X ; c_{1}, c_{2}}$ is not empty, then the moduli space consists of a single reduced point which represents a locally projective $\mathcal{A}$-module.

\section{Proof:}

Let $M$ and $N$ be torsion-free $\mathcal{A}$-modules of rank one defining points $[M]$ and $[N]$ in $M_{\mathcal{A} / X ; c_{1}, c_{2}}$. By (2.11) we know that $\chi_{\mathcal{A}}(M, N)$ depends only on the Chern classes of $N$ and not on $N$ itself. As $M$ and $N$ have the same Chern classes we get:

$$
\chi_{\mathcal{A}}(M, N)=\chi_{\mathcal{A}}(M, M)=-\left(v_{\mathcal{A}}(M), v_{\mathcal{A}}(M)\right)=2 .
$$

Therefore we must have either $\operatorname{Hom}_{\mathcal{A}}(M, N) \neq 0$, or $\operatorname{Hom}_{\mathcal{A}}(N, M) \neq 0$ by using Serre duality. Using (1.48) we see that in both cases $M$ and $N$ are isomorphic.

But it is known that $M_{\mathcal{A} / X ; c_{1}, c_{2}}$ is smooth (3.1, still to come) and that every connected component contains a locally projective $\mathcal{A}$-module (3.28, still to come).

Summing up: $M_{\mathcal{A} / X ; c_{1}, c_{2}}=\{[M]\},[M]$ is a reduced point and $M$ is locally projective.

\subsection{Two-dimensional moduli spaces}

Now we want to prove a result like (2.3) in our case, by using the $\mathcal{A}$-Mukai vector and the base change theorem for modules over an Azumaya algebra $\mathcal{A}$.

\section{Theorem 2.18:}

Assume $X$ is a $K 3$ or an abelian surface and let $\mathcal{A}$ be an Azumaya algebra on $X$. If $v_{\mathcal{A}}$ is a Mukai vector such that $\left(v_{\mathcal{A}}, v_{\mathcal{A}}\right)=0$, then either the moduli space is empty or $\operatorname{dim}\left(M_{\mathcal{A} / X ; c_{1}, c_{2}}\right)=2$. If $Y$ is a complete connected component in $M_{\mathcal{A} / X ; c_{1}, c_{2}}$, then $M_{\mathcal{A} / X ; c_{1}, c_{2}}=Y$, the moduli space is irreducible and all $\mathcal{A}$-modules are locally projective.

\section{Proof:}

Since the moduli space is smooth (3.1) and $Y$ is connected, the component must be irreducible (smooth and connected implies irreducible).

We fix a quasi-universal family $\mathcal{E}$ over $Y$, that is the restriction of a quasi-universal family on $M_{\mathcal{A} / X ; c_{1}, c_{2}}$ to $Y$, and denote the similitude by $s$.

We have the two projections $p: X \times Y \rightarrow X$ and $q: X \times Y \rightarrow Y$.

Let $[M]$ be an arbitrary point in the moduli space represented by an $\mathcal{A}$-module $M$. For any $y \in Y$ we have by (1.48):

$$
\operatorname{Hom}_{\mathcal{A}}\left(M, \mathcal{E}_{y}\right)= \begin{cases}0 & \text { if } M^{\oplus s} \nsucceq \mathcal{E}_{y} \\ k^{s} & \text { if } M^{\oplus s} \cong \mathcal{E}_{y}\end{cases}
$$

Furthermore Serre duality gives

$$
\operatorname{Ext}_{\mathcal{A}}^{2}\left(M, \mathcal{E}_{y}\right) \cong \operatorname{Hom}_{\mathcal{A}}\left(\mathcal{E}_{y}, M\right)^{\prime}= \begin{cases}0 & \text { if } M^{\oplus s} ¥ \mathcal{E}_{y} \\ k^{s} & \text { if } M^{\oplus s} \cong \mathcal{E}_{y}\end{cases}
$$


Since $\chi_{\mathcal{A}}\left(M, \mathcal{E}_{y}\right)=s \chi_{\mathcal{A}}(M, M)=0$ we see that:

$$
\operatorname{Ext}_{\mathcal{A}}^{1}\left(M, \mathcal{E}_{y}\right)= \begin{cases}0 & \text { if } M^{\oplus s} \nsucceq \mathcal{E}_{y} \\ k^{2 s} & \text { if } M^{\oplus s} \cong \mathcal{E}_{y}\end{cases}
$$

So if $[M] \notin Y$, then $\operatorname{Ext}_{\mathcal{A}}^{i}\left(M, \mathcal{E}_{y}\right)=0$ for all $y \in Y$ and $i=0,1,2$. Therefore we have

$$
\mathcal{E} x t_{p^{*} \mathcal{A}, q}^{i}\left(p^{*} M, \mathcal{E}\right)=0
$$

for $i=0,1,2$ by the base change theorem (1.77).

If $[M] \in Y$, then for $i=0,1,2$ we have $\operatorname{Ext}_{\mathcal{A}}^{i}\left(M, \mathcal{E}_{y}\right) \neq 0$ for just point $y_{0} \in Y$, namely $y_{0}=[M]$. Then lemma (1.78) applies and says that we have:

- $\mathcal{E} t_{p^{*} \mathcal{A}, q}^{i}\left(p^{*} M, \mathcal{E}\right)=0$ for $i=0,1$;

- $\mathcal{E} x t_{p^{*} \mathcal{A}, q}^{2}\left(p^{*} M, \mathcal{E}\right) \otimes k\left(y_{0}\right)=k\left(y_{0}\right)^{s}$ for $y_{0}=[M]$.

Particularly the sheaf $\mathcal{E} x t_{p^{*} \mathcal{A}, q}^{2}\left(p^{*} M, \mathcal{E}\right)$ does not vanish completely, so it is an Artinian sheaf of finite length on $Y$.

Lemma (1.85) computes the class

$$
a(M):=\operatorname{ch}\left(\mathcal{E} x t_{p^{*} \mathcal{A}, q}^{0}\left(p^{*} M, \mathcal{E}\right)-\mathcal{E} x t_{p^{*} \mathcal{A}, q}^{1}\left(p^{*} M, \mathcal{E}\right)+\mathcal{E} x t_{p^{*} \mathcal{A}, q}^{2}\left(p^{*} M, \mathcal{E}\right)\right)
$$

as an element of $H^{*}(Y, \mathbb{Q})$ and shows that it does not depend on $M$ but only on $\operatorname{ch}(M)$. If $[M] \notin Y$ we have $a(M)=0$ since $\mathcal{E} x t_{p^{*} \mathcal{A}, q}^{i}\left(p^{*} M, \mathcal{E}\right)=0$ for $i=0,1,2$ in this case. If $[M] \in Y$ we see that

$$
\int_{Y} a(M) \cdot t d(Y)=\chi\left(Y, \mathcal{E} x t_{p^{*} \mathcal{A}, q}^{2}\left(p^{*} M, \mathcal{E}\right)\right) \neq 0
$$

since $\mathcal{E} x t_{p^{*} \mathcal{A}, q}^{i}\left(p^{*} M, \mathcal{E}\right)=0$ for $i=0,1$ and $\mathcal{E} x t_{p^{*} \mathcal{A}, q}^{2}\left(p^{*} M, \mathcal{E}\right)$ is nontrivial and Artinian. But this can only happen if $a(M) \neq 0$. So if $[M] \in Y$ then $a(M) \neq 0$.

Consequently $a(N) \neq 0$ for all torsion free $\mathcal{A}$-modules $N$ of rank one with the same Chern classes as $M$, since $a(N)$ only depends on these classes. So we must have

$$
M_{\mathcal{A} / X ; c_{1}, c_{2}}=Y,
$$

because a sheaf $N$ in $M_{\mathcal{A} / X ; c_{1}, c_{2}} \backslash Y$ would have $a(N)=0$ which is not possible since all sheaves in $M_{\mathcal{A} / X ; c_{1}, c_{2}}$ have the same Chern classes.

For the last assertion, assume $M$ is a torsion-free $\mathcal{A}$-module of rank one with Chern classes $c_{1}$ and $c_{2}$. This module sits in the standard exact sequence:

$$
0 \longrightarrow M \longrightarrow M^{* *} \longrightarrow M^{* *} / M \longrightarrow 0 .
$$

Now $M^{* *}$ is a locally projective $\mathcal{A}$-module of rank one and has Chern classes $c_{1}$ and $c_{2}-l$, where $l$ is the length of $M^{* *} / M$ as an $\mathcal{O}_{X}$-module. But the length satifies

$$
l_{\mathcal{O}_{X}}\left(M^{* *} / M\right)=r l_{\mathcal{A}}\left(M^{* *} / M\right) .
$$

We see that we have $l=n r$ for some $n \in \mathbb{N}$. If $M$ is not locally projective then $n \geq 1$.

The module $M^{* *}$ defines a point in $M_{\mathcal{A} / X ; c_{1}, c_{2}-l}$ and we can compute that

$$
\operatorname{dim}\left(M_{\mathcal{A} / X ; c_{1}, c_{2}-l}\right)=2-2 l \text {. }
$$

Because $\mathcal{A}$ is nontrivial we have $r>1$, so that $r l>1$ impliying $\operatorname{dim}\left(M_{\mathcal{A} / X ; c_{1}, c_{2}-l}\right)<0$. But then $M_{\mathcal{A} / X ; c_{1}, c_{2}-l}$ is a non-empty smooth projective scheme of negative dimension, which is impossible. So we must have $l=0$, showing that $M \cong M^{* *}$.

Summing up: every torsion-free $\mathcal{A}$-module of rank one with Chern classes $c_{1}$ and $c_{2}$ is locally projective. 


\subsection{The case of an Azumaya Algebra on an abelian surface}

Assume $E_{1}$ and $E_{2}$ are smooth elliptic curves over $\mathbb{C}$.

\section{Definition 2.19:}

An isogeny $f: E_{1} \rightarrow E_{2}$ is a morphism of curves which maps the point at infinity $0_{1}$ in $E_{1}$ to the point at infinity $0_{2}$ in $E_{2}$.

Remark 2.20:

Since an elliptic curve is also an algebraic group, this definition of isogeny already implies that an isogeny is a morphism of algebraic groups.

As $E_{1}$ is a complete nonsingular curve, there are only two possibilities for the image of $E_{1}$ under $f$ due to the following proposition:

Proposition 2.21 ([Har77, Proposition II.6.8]):

Let $f: X \rightarrow Y$ be a morphism where $X$ is a complete nonsingular curve over $\mathbb{C}$ and $Y$ is any curve over $\mathbb{C}$. Then either $f(X)$ is a point or $f(X)=Y$. In the second case $\mathbb{C}(X)$ is a finite extension of $\mathbb{C}(Y), f$ is a finite morphism and $Y$ is also complete.

\section{Example 2.22:}

Look at the multiplication by n map $[n]: E_{1} \rightarrow E_{1}, P \mapsto n P$. Then we certainly have $[n]\left(0_{1}\right)=0_{1}$ so that $[n]$ is a nontrivial isogeny.

\section{Remark 2.23:}

If $f: E_{1} \rightarrow E_{2}$ is a nontrivial isogeny, then this morphism induces a map

$$
f^{*}: \operatorname{Pic}^{0}\left(E_{2}\right) \rightarrow \operatorname{Pic}^{0}\left(E_{1}\right)
$$

which yields the so-called dual isogeny $\hat{f}: E_{2} \rightarrow E_{1}$ by noticing that $E_{i} \cong P i c^{0}\left(E_{i}\right)$. With the help of the dual isogeny one can show that being isogenous is an equivalence relation.

So if $E_{1}$ and $E_{2}$ are non-isogenous elliptic curves, there is only the zero isogeny between them, which maps the whole of $E_{1}$ to $0_{2}$ in $E_{2}$.

We end this short recollection about isogenies with this lemma, which will be used later.

\section{Lemma 2.24:}

Assume $E_{1}$ and $E_{2}$ are two non-isogenous elliptic curves, then $E_{1}\left(\mathbb{C}\left(E_{2}\right)\right)=E_{1}(\mathbb{C})$.

\section{Proof:}

Assume we have a $\mathbb{C}\left(E_{2}\right)$-valued point on $E_{1}$, that is a morphism $f_{\eta}: \operatorname{Spec}\left(\mathbb{C}\left(E_{2}\right)\right) \rightarrow E_{1}$, here $\eta=\operatorname{Spec}\left(\mathbb{C}\left(E_{2}\right)\right)$ denotes the generic point of $E_{2}$. We choose a closed point $x \in E_{2}$, that is $x$ has codimension one in $E_{2}$.

Since $E_{1}$ is proper, the morphism $f_{\eta}$ extends uniquely to a morphism $f_{x}: \operatorname{Spec}\left(\mathcal{O}_{E_{2}, x}\right) \rightarrow E_{1}$ by the valuative criterion of properness. But as these curves are Noetherian and of finite type over $\mathbb{C}$, there is an open subset $U \subset E_{2}$ containing $x$ such the morphism $f_{x}$ extends uniquely to a morphism $f_{U}: U \rightarrow E_{1}$.

Consequently there are finitely many points on $E_{2}$ for which this map is not defined. But then 
$f_{U}$ extends uniquely to a morphism $f: E_{2} \rightarrow E_{1}$, compatible with $f_{\eta}: \operatorname{Spec}\left(\mathbb{C}\left(E_{2}\right)\right) \rightarrow E_{1}$, see [Liu02, Corollary 4.1.17].

Possibly using an appropriate translation, we may assume the map $f: E_{2} \rightarrow E_{1}$ preserves the origins. But then this map is either an isogeny or constant. Since $E_{1}$ and $E_{2}$ are non-isogenous, the morphism $f$ constructed from $f_{\eta}$ must be constant.

That is we have a factorization of $f$ through $\operatorname{Spec}(\mathbb{C})$ :

$$
E_{2} \longrightarrow \operatorname{Spec}(\mathbb{C}) \longrightarrow E_{1},
$$

but then $f_{\eta}$ also factors through $\operatorname{Spec}(\mathbb{C})$. This shows that the $\mathbb{C}\left(E_{2}\right)$-valued point is in fact a $\mathbb{C}$-valued point.

We are interested in the abelian surface defined by $X:=E_{1} \times E_{2}$. We remark that the canonical line bundle $\omega_{X}$ is trivial, i.e $K_{X}=0$. The cohomology groups of the structure sheaf are given by $H^{0}\left(\mathcal{O}_{X}\right)=H^{2}\left(\mathcal{O}_{X}\right)=k$ and $H^{1}\left(\mathcal{O}_{X}\right)=k^{2}$.

Now choose two-torsion line bundles $L_{i} \in \operatorname{Pic}\left(E_{i}\right)$ for $i=1,2$, this means $L_{i}$ satisfies $L_{i}^{2} \cong \mathcal{O}_{E_{i}}$. Define $I:=\pi_{1}^{*} L_{1}$ and $J:=\pi_{2}^{*} L_{2}$, where $\pi_{i}: X \rightarrow E_{i}$ are the projections. Then $I, J$ and $K=I \otimes J$ are two-torsion line bundles on $X$. So we can fix isomorphisms $\alpha_{I}: I^{2} \stackrel{\sim}{\longrightarrow} \mathcal{O}_{X}$ and $\alpha_{J}: J^{2} \stackrel{\sim}{\longrightarrow} \mathcal{O}_{X}$. Then we define a locally free sheaf $\mathcal{A}$ of rank four on $X$ by:

$$
\mathcal{A}=\mathcal{O}_{X} \oplus I \oplus J \oplus K
$$

Using $\alpha_{I}$ and $\alpha_{J}$ we can define the structure of a quaternion algebra on $\mathcal{A}$ :

- if $l_{1}, l_{2} \in \Gamma(U, I)$ we define $l_{1} \cdot l_{2}=\alpha_{I}\left(l_{1} l_{2}\right) \in \mathcal{O}_{X}(U)$ and do the same for $J$ and $K$;

- for $l \in \Gamma(U, I)$ and $m \in \Gamma(U, J)$ we define $l \cdot m=-m \cdot l$.

With this definition we have $\alpha_{K}=-\alpha_{I} \otimes \alpha_{J}$.

To see that $\mathcal{A}$ is an Azumaya algebra we note that we can find local sections $i$ and $j$ for $I$ and $J$ over an open set $U$ in the étale topology such that $\alpha_{I}\left(i^{2}\right)=1$ and $\alpha_{J}\left(j^{2}\right)=1$. Defining $k=i \otimes j$, we see that $\{1, i, j, k\}$ with $i j=k, j i=-k$ and $i^{2}=j^{2}=-k^{2}=1$ is a local basis for $\mathcal{A}$ on $U$. But then there is an isomorphism

$$
\mathcal{A} \otimes \mathcal{O}_{X} \mathcal{O}_{U} \cong M_{2}\left(\mathcal{O}_{U}\right) .
$$

This can be seen like in the case of a field $F$, where such an isomorphism is given by mapping a basis $\{1, i, j, k\}$ with the properties described above via

$$
1 \mapsto\left(\begin{array}{cc}
1 & 0 \\
0 & 1
\end{array}\right), i \mapsto\left(\begin{array}{cc}
1 & 0 \\
0 & -1
\end{array}\right), j \mapsto\left(\begin{array}{cc}
0 & 1 \\
1 & 0
\end{array}\right) \text { and } k \mapsto\left(\begin{array}{cc}
0 & 1 \\
-1 & 0
\end{array}\right)
$$

to $M_{2}(F)$. One checks that this is indeed an isomorphism and transfers this to the global situation.

Now we want to see that $\mathcal{A}$ is a nontrivial Azumaya algebra on $X$, which means its Brauer class $[\mathcal{A}] \in \operatorname{Br}(X)$ is nontrivial. By the sequence $(1.4)$ the map $[\mathcal{A}] \rightarrow\left[\mathcal{A}_{\eta}\right]$ from $\operatorname{Br}(X)$ to $\operatorname{Br}(\mathbb{C}(X))$ is injective, so it is enough to show that $\left[\mathcal{A}_{\eta}\right]$ is nontrivial in $\operatorname{Br}(\mathbb{C}(X))$. But

$$
\mathcal{A}_{\eta}=\left(\mathcal{O}_{X} \oplus I \oplus J \oplus K\right)_{\eta}=\mathcal{O}_{X, \eta} \oplus I_{\eta} \oplus J_{\eta} \oplus K_{\eta}
$$

and $\mathcal{O}_{X, \eta}=I_{\eta}=J_{\eta}=K_{\eta}=\mathbb{C}(X)$.

If $I=\mathcal{O}_{X}(D)$, then $I^{2} \cong \mathcal{O}_{X}$ means $2 D=\operatorname{div}(f)$ for some $f \in \mathbb{C}(X)^{\times}$. So 


$$
I^{2}=\mathcal{O}_{X}(\operatorname{div}(f))=\frac{1}{f} \mathcal{O}_{X}
$$

and $\alpha_{I}$ is given by multiplication with $f$, see for example [Har77, Proposition II.6.13]. Likewise $J^{2}=\mathcal{O}_{X}(\operatorname{div}(g))$ implies $J^{2}=\frac{1}{g} \mathcal{O}_{X}$ and $\alpha_{J}$ is multiplication with $g$. This shows that $\mathcal{A}_{\eta}$ is the quaternion algebra $(f, g)_{2}$, that is the $\mathbb{C}(X)$-algebra generated by elements $i$ and $j$ with $i^{2}=f$, $j^{2}=g$ and $i j=-j i$.

It remains to show that $\left[(f, g)_{2}\right]$ is nontrivial in $\operatorname{Br}(\mathbb{C}(X))$. But this is true, due to the following proposition:

Proposition 2.25 ([CT02, Proposition 11]):

Assume $E_{1}$ and $E_{2}$ are non-isogenous elliptic curves and choose $f \in \mathbb{C}\left(E_{1}\right)^{\times}$and $g \in \mathbb{C}\left(E_{2}\right)^{\times}, f$ and $g$ no squares, such that $\operatorname{div}(f)$ and $\operatorname{div}(g)$ are multiples of two. Then the quaternion algebra $(f, g)_{2}$ is nontrivial in $\operatorname{Br}\left(\mathbb{C}\left(E_{1} \times E_{2}\right)\right)$.

\section{Remark 2.26:}

We will outline the basic ideas behind the proof of this proposition:

Assume $k$ is an arbitrary field of characteristic different from two and let $E$ be an elliptic curve over $k$ whose two-torsion points are rational. Fix an isomorphism $\gamma:(\mathbb{Z} / 2 \mathbb{Z})^{2} \cong E(k)[2]$.

Now define $B r^{0}(E):=\operatorname{ker}\left(B r(E) \stackrel{\epsilon_{0}^{*}}{\rightarrow} B r(k)\right)$, where $\epsilon_{0}: \operatorname{Spec}(k) \rightarrow E$ is the inclusion of the origin $0_{E} \in E$, that is $\epsilon_{0}^{*}$ is the restriction of an Azumaya algebra to the origin. Then one has a canonical isomorphism

$$
B r^{0}(E) \cong H^{1}(k, E),
$$

where $H^{i}(k,-)$ is Galois cohomology, see [Wit04, Lemma 2.1]. The Kummer sequence

$$
0 \longrightarrow E[2] \longrightarrow E \stackrel{[2]}{\longrightarrow} E \longrightarrow 0
$$

gives a long exact sequence in Galois cohomology, which can be split in parts to give the following short exact sequence:

$$
0 \longrightarrow E(k) / 2 E(k) \longrightarrow H^{1}(k, E[2]) \longrightarrow H^{1}(k, E)[2] \longrightarrow 0 .
$$

Using the isomorphism $\gamma, H^{1}(k, \mathbb{Z} / n \mathbb{Z}) \cong k^{\times} /\left(k^{\times}\right)^{n}$ and $\operatorname{Br}^{0}(E)=H^{1}(k, E)$ gives the following sequence

$$
0 \longrightarrow E(k) / 2 E(k) \longrightarrow\left(k^{\times} / k^{\times 2}\right)^{2} \stackrel{\phi}{\longrightarrow}{ }_{2} B r^{0}(E) \longrightarrow 0 .
$$

where ${ }_{2} B r^{0}(E)$ denotes the two-torsion part of $B r^{0}(E)$.

If we take $E$ to be our elliptic curve $E_{1}$ and take $k$ to be the function field $\mathbb{C}\left(E_{2}\right)$ of the other curve, then $(2.24)$ says that $E(k)=E_{1}\left(\mathbb{C}\left(E_{2}\right)\right)=E_{1}(\mathbb{C})$. But since $\mathbb{C}$ is algebraically closed we have $E_{1}(\mathbb{C})=2 E_{1}(\mathbb{C})$, implying $E(k) / 2 E(k)=0$.

Furthermore we have $\operatorname{Br}\left(\mathbb{C}\left(E_{2}\right)\right)=0$, see (A.5), so $\operatorname{Br}^{0}\left(E_{1}\right)=\operatorname{Br}\left(E_{1}\right)$.

Thus we have an isomorphism

$$
\left(k^{\times} / k^{\times 2}\right)^{2} \cong{ }_{2} \operatorname{Br}\left(E_{1}\right)
$$

where $E_{1}$ is defined over $k=\mathbb{C}\left(E_{2}\right)$. Now any class $g \in \mathbb{C}\left(E_{2}\right)^{\times}$described in the lemma actually comes from a two-torsion line bundle on $E_{2}$, which are classified by $H_{\text {êt }}^{1}\left(E_{2}, \mathbb{Z} / 2 \mathbb{Z}\right)$. So restricting to these classes gives an injection $\left(H_{\text {êt }}^{1}\left(E_{2}, \mathbb{Z} / 2 \mathbb{Z}\right)\right)^{2} \hookrightarrow{ }_{2} \operatorname{Br}\left(E_{1}\right)$. But with the help of this injection and the map $\phi$ one can then show that 


$$
H_{\text {êt }}^{1}\left(E_{1}, \mathbb{Z} / 2 \mathbb{Z}\right) \otimes H_{\text {êt }}^{1}\left(E_{2}, \mathbb{Z} / 2 \mathbb{Z}\right) \cong{ }_{2} B r\left(E_{1} \times E_{2}\right) .
$$

The result then follows because one has an explicit description of the map $\phi$ involving the algebras $(a, b)_{2}$ see [Wit04, Proposition 2.2].

Using the Künneth formula for $X=E_{1} \times E_{2}$ shows that we have

$$
H^{0}(X, \mathcal{A})=H^{2}(X, \mathcal{A})=k \text { and } H^{1}(X, \mathcal{A})=k^{2},
$$

so the Euler characteristic vanishes: $\chi(X, \mathcal{A})=0$. Furthermore we have

$$
c_{1}(\mathcal{A})=0 \text { and } c_{2}(\mathcal{A})=0 .
$$

We would like to study the moduli space $M_{\mathcal{A} / X ; c_{1}, c_{2}}$ of torsion-free $\mathcal{A}$-module of rank one with Chern classes $c_{1}=0$ and $c_{2}=0$. We immediately see that $M_{\mathcal{A} / X ; 0,0}$ is not empty because it contains $\mathcal{A}$. If $M$ is an $\mathcal{A}$-module with the prescribed Chern classes then we have

$$
v_{\mathcal{A}}(M)=(2,0,0)
$$

by (2.13) and hence $\left(v_{\mathcal{A}}(M), v_{\mathcal{A}}(M)\right)=0$. Using (2.15) we obtain

$$
\operatorname{dim}\left(M_{\mathcal{A} / X ; 0,0}\right)=2 .
$$

As the space is not empty it contains at least one connected component of dimension two. Because the space is smooth, see (3.1), this component is in fact irreducible, hence a smooth projective surface. We would like to describe this component completely and use (2.18) to show that this component is in fact the whole moduli space. To do this we need the following lemmas (for the rest of this chapter $\otimes$ always means the tensor product over $\mathcal{O}_{X}$ ):

\section{Lemma 2.27:}

Assume $G$ is the Kleinian four-group $\left\{\mathcal{O}_{X}, I, J, K\right\} \subset \operatorname{Pic}(X)$. Then for $L \in \operatorname{Pic}(X)$ we have: $\mathcal{A} \otimes L \cong \mathcal{A}$ as $\mathcal{O}_{X}$-modules if and only if $L \in G$.

\section{Proof:}

Since $\mathcal{A}$ is a direct sum of line bundles as an $\mathcal{O}_{X}$-module we see:

$$
\begin{aligned}
\mathcal{A} & =\mathcal{O}_{X} \oplus I \oplus J \oplus K \\
\mathcal{A} \otimes L & =L \oplus(I \otimes L) \oplus(J \otimes L) \oplus(K \otimes L) .
\end{aligned}
$$

If $L \in G$ we immediately get that $\mathcal{A} \otimes L \cong \mathcal{A}$.

Assume $\mathcal{A} \otimes L \cong \mathcal{A}$ holds. Since the Krull-Schmidt theorem is true for locally free sheaves, see [Ati56, Theorem 5.3], one direct summand of $\mathcal{A} \otimes L$ must be isomorphic to the direct summand $\mathcal{O}_{X}$ in $\mathcal{A}$. So we must have

$$
L \cong \mathcal{O}_{X}, I \otimes L \cong \mathcal{O}_{X}, J \otimes L \cong \mathcal{O}_{X} \text { or } K \otimes L \cong \mathcal{O}_{X}
$$

Since $I, J$ and $K$ are two-torsion line bundles we can multiply each isomorphism with the respective line bundle and get the desired result.

\section{Corollary 2.28:}

Assume $G$ is the Kleinian four-group $\left\{\mathcal{O}_{X}, I, J, K\right\} \subset \operatorname{Pic}(X)$ and $L_{i} \in \operatorname{Pic}(X)$ for $i=1,2$. Then we have $\mathcal{A} \otimes L_{1} \cong \mathcal{A} \otimes L_{2}$ as $\mathcal{O}_{X}$-modules if and only if $L_{1} \otimes L_{2}^{-1} \in G$. 


\section{Proof:}

We have $\mathcal{A} \otimes L_{1} \cong \mathcal{A} \otimes L_{2}$ if and only if $\mathcal{A} \cong \mathcal{A} \otimes\left(L_{1} \otimes L_{2}^{-1}\right)$. Now one can use (2.27).

\section{Corollary 2.29:}

Assume $L_{1}$ and $L_{2}$ are line bundles on $X$. Then we have $\mathcal{A} \otimes L_{1} \cong \mathcal{A} \otimes L_{2}$ as $\mathcal{O}_{X}$-modules if and only if $\mathcal{A} \otimes L_{1} \cong \mathcal{A} \otimes L_{2}$ as $\mathcal{A}$-modules.

\section{Proof:}

If these $\mathcal{A}$-line bundles are isomorphic as $\mathcal{A}$-modules, then they are isomorphic as $\mathcal{O}_{X}$-modules using the forgetful functor from $\mathcal{A}$-modules to $\mathcal{O}_{X}$-modules.

Assume $\mathcal{A} \otimes L_{1} \cong \mathcal{A} \otimes L_{2}$ as $\mathcal{O}_{X}$-modules. Then by (2.28) we have

$$
H^{0}\left(X, \mathcal{A} \otimes L_{1} \otimes L_{2}^{-1}\right)=H^{0}(X, \mathcal{A})=k .
$$

But then we see that

$$
\begin{aligned}
\operatorname{Hom}_{\mathcal{A}}\left(\mathcal{A} \otimes L_{2}, \mathcal{A} \otimes L_{1}\right) & =\operatorname{Hom}_{\mathcal{A}}\left(\mathcal{A}, \mathcal{A} \otimes L_{1} \otimes L_{2}^{-1}\right) \\
& =\operatorname{Hom}_{\mathcal{O}_{X}}\left(\mathcal{O}_{X}, \mathcal{A} \otimes L_{1} \otimes L_{2}^{-1}\right) \\
& =H^{0}\left(X, \mathcal{A} \otimes L_{1} \otimes L_{2}^{-1}\right) \\
& =k .
\end{aligned}
$$

Now (1.48) shows that $\mathcal{A} \otimes L_{1} \cong \mathcal{A} \otimes L_{2}$ as $\mathcal{A}$-modules.

We use lemma (1.79) to see that for any $\mathcal{A}$-module $M$ and any line bundle $L \in P i c^{0}(X)$ we have:

- $c_{1}\left(M \otimes \mathcal{O}_{X} L\right)=c_{1}(M)$;

- $c_{2}\left(M \otimes \mathcal{O}_{X} L\right)=c_{2}(M)$.

Since $E_{1}$ and $E_{2}$ are non-isogenous, we deduce from the proof of [BL04, Theorem 11.5.1] that

$$
\operatorname{Pic}(X)=\pi_{1}^{*} \operatorname{Pic}\left(E_{1}\right) \times \pi_{2}^{*} \operatorname{Pic}\left(E_{2}\right) .
$$

So the group $G=\left\{\mathcal{O}_{X}, I, J, K\right\}$ is given as the product $H_{1} \times H_{2}$ where $H_{1}=\langle I\rangle$ and $\mathrm{H}_{2}=<J>$.

Furthermore we have

$$
\operatorname{Pic}^{0}(X)=\pi_{1}^{*} \operatorname{Pic}^{0}\left(E_{1}\right) \times \pi_{2}^{*} \operatorname{Pic}^{0}\left(E_{2}\right),
$$

which is true for any two curves. So $\operatorname{Pic}^{0}(X)$ can be identified with $X$ itself, since $\operatorname{Pic}^{0}(E) \cong E$ for an elliptic curve.

We see that in fact $H_{i} \subset \pi_{i}^{*} \operatorname{Pic}^{0}\left(E_{i}\right)$ so that $G$ also acts on $\operatorname{Pic}^{0}(X)$ and the action of $G$ is exactly the product of the actions of the $H_{i}$ on $\pi_{i}^{*} P i c^{0}\left(E_{i}\right)$. The action of $G$ on $\operatorname{Pic}^{0}(X)$ is obviously free.

As a consequence $G$ also acts freely on $X$. As $G$ is finite and $X$ is projective this implies that $X / G$ exists and that it is smooth, see [Mum74, Appendix to §6]. It is also known that $X / G$ is again projective, see for example [Har92, Lecture 10].

Since the tensor product with a line bundle in $\operatorname{Pic}^{0}(X)$ preserves the Chern classes, we get a map

$$
\operatorname{Pic}^{0}(X) \longrightarrow M_{\mathcal{A} / X ; 0,0}
$$


which maps a line bundle $L$ to the isomorphism class of $\mathcal{A} \otimes_{\mathcal{O}_{X}} L$. Putting everything together we have a map

$$
X / G \longrightarrow M_{\mathcal{A} / X ; 0,0}
$$

which sends the equivalence class $[L]$ of a line bundle in $X / G$ to the isomorphism class of $\mathcal{A} \otimes_{\mathcal{O}_{X}} L$. By (2.28) this map is well defined and satisfies the following property: if $\left[L_{1}\right] \neq\left[L_{2}\right]$ then $\mathcal{A} \otimes \mathcal{O}_{X} L_{1} \neq \mathcal{A} \otimes \mathcal{O}_{X} L_{2}$.

Thus we have an embedding

$$
X / G \hookrightarrow M_{\mathcal{A} / X ; 0,0} .
$$

So $X / G$ is a complete connected component of the moduli space. But then by (2.18) we have

$$
X / G=M_{\mathcal{A} / X ; 0,0} .
$$

We can also describe the quotient $X / G$ more explicitly: Since $X=E_{1} \times E_{2}, G=H_{1} \times H_{2}$ and the action of $G$ is exactly the product of the actions of the $H_{i}$ we see that in fact we have

$$
X / G=\left(E_{1} \times E_{2}\right) /\left(H_{1} \times H_{2}\right) \cong E_{1} / H_{1} \times E_{2} / H_{2} .
$$

Now we use the following proposition:

Proposition 2.30 ([Sil86, Proposition III.4.12]):

Assume $E$ is an elliptic curve and $G$ is a finite subgroup of $E$. Then there exists a unique elliptic curve $E^{\prime}$ and an isogeny $f: E \rightarrow E^{\prime}$ such that $\operatorname{ker}(f)=G$ and $\operatorname{deg}(f)=|G|$.

So there are two elliptic curves $E_{1}^{\prime}$ and $E_{2}^{\prime}$ such that $E_{i}$ is isogenous to $E_{i}^{\prime}$ for $i=1,2$. Since being isogenous is an equivalence relation we see that $E_{1}^{\prime}$ and $E_{2}^{\prime}$ are also non-isogenous like $E_{1}$ and $E_{2}$. Summing up everything, we proved the following theorem:

\section{Theorem 2.31:}

Assume $X$ is a smooth abelian surface, given as the product of two smooth non-isogneous elliptic curves $E_{1}$ and $E_{2}$. Let $\mathcal{A}$ be the Azumaya algebra on $X$ defined by (8). Then the moduli space $M_{\mathcal{A} / X ; 0,0}$ of torsion-free $\mathcal{A}$-modules of rank one with Chern classes $c_{1}=0$ and $c_{2}=0$ is a smooth projective surface, which is itself a product of non-isogenous elliptic curves $E_{1}^{\prime}$ and $E_{2}^{\prime}$ such that $E_{i}^{\prime}$ is isogenous to $E_{i}$ via an isogeny of degree two for $i=1,2$. 


\section{Moduli spaces over the projective plane: del Pezzo orders}

\subsection{Smoothness}

In [HS05] the following theorem is one of the main results:

\section{Theorem 3.1:}

Assume $X$ is a $K 3$ or abelian surface and $\mathcal{A}$ is an Azumaya algebra on $X$. Then the moduli space $M_{\mathcal{A} / X ; c_{1}, c_{2}}$ is smooth and one has

$$
\operatorname{dim}\left(M_{\mathcal{A} / X ; c_{1}, c_{2}}\right)=\frac{1}{r^{2}} \Delta-c_{2}(\mathcal{A})-r^{2} \chi\left(\mathcal{O}_{X}\right)+2
$$

where $r k(\mathcal{A})=r^{2}$ and $\Delta=2 r^{2} c_{2}-\left(r^{2}-1\right) c_{1}^{2}$ is the discriminant of the $\mathcal{A}$-modules.

In this section we will prove a similar result assuming $\mathcal{A}$ is a terminal del Pezzo order on $\mathbb{P}^{2}$.

\section{Lemma 3.2:}

Assume $X$ is a smooth projective surface and $\mathcal{A}$ is a terminal order on $X$. If $M$ is a torsion-free $\mathcal{A}$-module of rank one, then so is $\omega_{\mathcal{A}} \otimes_{\mathcal{A}} M$.

\section{Proof:}

Because $M$ and $\omega_{\mathcal{A}}$ are torsion-free $\mathcal{A}$-modules of rank one, the generic stalks $M_{\eta}$ and $\omega_{\mathcal{A}, \eta}$ are one dimensional vector spaces over the division $\operatorname{ring} \mathcal{A}_{\eta}$. This shows that $\omega_{\mathcal{A}, \eta} \otimes_{\mathcal{A}_{\eta}} M_{\eta}$ is also a one dimensional vector space over $\mathcal{A}_{\eta}$, so it is a simple $\mathcal{A}_{\eta}$-module. We conclude that $\omega_{\mathcal{A}} \otimes_{\mathcal{A}} M$ is of rank one.

Since $\omega_{\mathcal{A}} \otimes_{\mathcal{A}} M$ is coherent, it remains to show that $\omega_{\mathcal{A}} \otimes_{\mathcal{A}} M$ is a torsion-free $\mathcal{O}_{X}$-module, meaning we have to show that for every point $p \in X$ the $\mathcal{O}_{X, p}$-module $\left(\omega_{\mathcal{A}} \otimes_{\mathcal{A}} M\right)_{p}=\omega_{\mathcal{A}, p} \otimes_{\mathcal{A}_{p}} M_{p}$ is torsion-free.

As $M$ is coherent and torsion-free we know that $M_{p}$ is a torsion-free $\mathcal{O}_{X, p}$-module for every $p \in X$. We thus get an embedding:

$$
M_{p} \hookrightarrow M_{p} \otimes_{\mathcal{O}_{X, p}} K,
$$

where $K=\operatorname{Quot}\left(\mathcal{O}_{X, p}\right)$ is the field of fractions of $\mathcal{O}_{X, p}$.

Now $\omega_{\mathcal{A}, p}$ is a finitely generated free $\mathcal{O}_{X, p}$-module, hence it is a projective $\mathcal{A}_{p}$-module by (1.13). So it is especially a flat $\mathcal{A}_{p}$-module. We tensor (9) with $\omega_{\mathcal{A}, p}$ over $\mathcal{A}_{p}$ to get an injection:

$$
\omega_{\mathcal{A}, p} \otimes_{\mathcal{A}_{p}} M_{p} \hookrightarrow \omega_{\mathcal{A}, p} \otimes_{\mathcal{A}_{p}}\left(M_{p} \otimes_{\mathcal{O}_{X, p}} K\right) .
$$

As the tensor product is associative we see:

$$
\omega_{\mathcal{A}, p} \otimes_{\mathcal{A}_{p}}\left(M_{p} \otimes_{\mathcal{O}_{X, p}} K\right) \cong\left(\omega_{\mathcal{A}, p} \otimes_{\mathcal{A}_{p}} M_{p}\right) \otimes_{\mathcal{O}_{X, p}} K .
$$

But the latter module is a vector space over $K$, hence it is a torsion-free $\mathcal{O}_{X, p}$-module.

This implies that $\omega_{\mathcal{A}, p} \otimes_{\mathcal{A}_{p}} M_{p}$ is a torsion-free $\mathcal{O}_{X, p}$-module as it is a submodule of a torsion-free module.

\section{Remark 3.3:}

These modules appear when applying Serre duality for $\mathcal{A}$-modules. This lemma shows that all results from section (1.4) are also true for $\mathcal{A}$-modules of the form $\omega_{\mathcal{A}} \otimes_{\mathcal{A}} M$ for some torsion-free $\mathcal{A}$-module $M$ of rank one. 


\section{Lemma 3.4:}

Assume $\mathcal{A}$ is a terminal del Pezzo order on $\mathbb{P}^{2}$. If $M$ and $N$ are torsion-free $\mathcal{A}$-modules of rank one with $c_{1}(M)=c_{1}(N)$, then $\operatorname{Ext}_{\mathcal{A}}^{2}(M, N)=0$.

\section{Proof:}

Using Serre duality we see that:

$$
\operatorname{Ext}_{\mathcal{A}}^{2}(M, N) \cong \operatorname{Hom}_{\mathcal{A}}\left(N, \omega_{\mathcal{A}} \otimes_{\mathcal{A}} M\right)^{\prime} .
$$

So assume $\operatorname{Hom}_{\mathcal{A}}\left(N, \omega_{\mathcal{A}} \otimes_{\mathcal{A}} M\right) \neq 0$ and pick a nontrivial element $f \in \operatorname{Hom}_{\mathcal{A}}\left(N, \omega_{\mathcal{A}} \otimes_{\mathcal{A}} M\right)$. Then by (1.47) $f$ is injective. So we get an exact sequence:

$$
0 \longrightarrow N \stackrel{f}{\longrightarrow} \omega_{\mathcal{A}} \otimes_{\mathcal{A}} M \longrightarrow \mathcal{Q} \longrightarrow 0 .
$$

Now $D:=c_{1}\left(\omega_{\mathcal{A}} \otimes_{\mathcal{A}} M\right)-c_{1}(N)$ should be an effective divisor, but we can compute this difference. We first note that:

$$
\omega_{\mathcal{A}} \otimes_{\mathcal{A}} M=\mathcal{H o m}_{\mathcal{O}_{X}}\left(\mathcal{A}, \omega_{\mathbb{P}^{2}}\right) \otimes_{\mathcal{A}} M \cong \omega_{\mathbb{P}^{2}} \otimes_{\mathcal{O}_{X}} \mathcal{A}^{*} \otimes_{\mathcal{A}} M
$$

This shows that

$$
c_{1}\left(\omega_{\mathcal{A}} \otimes_{\mathcal{A}} M\right)=r k(\mathcal{A}) c_{1}\left(\omega_{\mathbb{P}^{2}}\right)+c_{1}\left(\mathcal{A}^{*} \otimes_{\mathcal{A}} M\right) .
$$

Using (1.84) we get:

$$
c_{1}\left(\omega_{\mathcal{A}} \otimes_{\mathcal{A}} M\right)=r k(\mathcal{A}) c_{1}\left(\omega_{\mathbb{P}^{2}}\right)+c_{1}(M)-2 c_{1}(\mathcal{A}) .
$$

Putting everything together gives:

$$
\begin{aligned}
D & =r k(\mathcal{A}) c_{1}\left(\omega_{\mathbb{P}^{2}}\right)+c_{1}(M)-2 c_{1}(\mathcal{A})-c_{1}(N) \\
& =r k(\mathcal{A}) c_{1}\left(\omega_{\mathbb{P}^{2}}\right)-2 c_{1}(\mathcal{A})
\end{aligned}
$$

By lemma $(1.83)$ we have $c_{1}(\mathcal{A})=-\frac{r k(\mathcal{A})}{2} \sum_{i=1}^{l}\left(1-\frac{1}{e_{i}}\right) C_{i}$ and as $c_{1}\left(\omega_{\mathbb{P}^{2}}\right)=K_{\mathbb{P}^{2}}$, we finally see:

$$
\begin{aligned}
D & =r k(\mathcal{A})\left(K_{\mathbb{P}^{2}}+\sum_{i=1}^{l}\left(1-\frac{1}{e_{i}}\right) C_{i}\right) \\
& =r k(\mathcal{A}) K_{\mathcal{A}} .
\end{aligned}
$$

But $\mathcal{A}$ is a del Pezzo order, so the canonical divisor $-K_{\mathcal{A}}$ is ample. As $\mathbb{P}^{2}$ has Picard number one, every non-zero effective divisor is ample, see [Har77, Example II.7.6.1].

So $D$ cannot be effective, because if it were it also had to be ample, which is impossible since $-D$ is ample. Consequently such an element $f$ cannot exist.

We conclude that $\operatorname{Hom}_{\mathcal{A}}\left(N, \omega_{\mathcal{A}} \otimes_{\mathcal{A}} M\right)=0$, which implies that $\operatorname{Ext}_{\mathcal{A}}^{2}(M, N)=0$.

\section{Remark 3.5:}

Regarding $\mathcal{A}$ as a torsion-free $\mathcal{A}$-module of rank one over itself, the previous lemma (3.4) and the local-to-global spectral sequence show that for every terminal del Pezzo order $\mathcal{A}$ on $\mathbb{P}^{2}$ :

$$
H^{2}\left(\mathbb{P}^{2}, \mathcal{A}\right)=\operatorname{Ext}_{\mathcal{A}}^{2}(\mathcal{A}, \mathcal{A})=0 .
$$


Theorem 3.6:

Assume $\mathcal{A}$ is a terminal del Pezzo order on $\mathbb{P}^{2}$, then the moduli space $M_{\mathcal{A} / \mathbb{P}^{2} ; c_{1}, c_{2}}$ of torsion-free $\mathcal{A}$-modules of rank one with Chern classes $c_{1}$ and $c_{2}$ is smooth.

\section{Proof:}

Assume $M$ is a torsion-free $\mathcal{A}$-module of rank one, which defines a point in $M_{\mathcal{A} / \mathbb{P}^{2} ; c_{1}, c_{2}}$.

We have to show that all obstruction classes in $\operatorname{Ext}_{\mathcal{A}}^{2}(M, M)$ vanish. But by the previous lemma (3.4) we even have $\operatorname{Ext}_{\mathcal{A}}^{2}(M, M)=0$ in this case. So all obstruction classes must vanish and $M_{\mathcal{A} / \mathbb{P}^{2} ; c_{1}, c_{2}}$ is smooth.

\section{Proposition 3.7:}

The dimension of the moduli space $M_{\mathcal{A} / \mathbb{P}^{2} ; c_{1}, c_{2}}$ at a point depends only on the Chern classes $c_{1}$ and $c_{2}$.

\section{Proof:}

The Kodaira-Spencer map gives an isomorphism for a torsion-free $\mathcal{A}$-module $M$ representing a point in the moduli space:

$$
T_{[M]} M_{\mathcal{A} / \mathbb{P}^{2} ; c_{1}, c_{2}} \cong \operatorname{Ext}_{\mathcal{A}}^{1}(M, M) .
$$

As the moduli space is smooth, its dimension at $[M]$ is the dimension of its tangent space.

The $\mathcal{A}$-Euler characteristic $\chi_{\mathcal{A}}(M, M)=\sum_{i=0}^{2}(-1)^{i} \operatorname{dim}\left(\operatorname{Ext}_{\mathcal{A}}^{i}(M, M)\right)$ can be computed just using the Chern classes of $M$, see (2.14).

We know that:

- $\operatorname{Ext}_{\mathcal{A}}^{0}(M, M)=\operatorname{End}_{\mathcal{A}}(M)=k$ from (1.49);

- $\operatorname{Ext}_{\mathcal{A}}^{2}(M, M)=0$ from (3.4).

So we see that $\operatorname{dim}\left(M_{\mathcal{A} / \mathbb{P}^{2} ; c_{1}, c_{2}}\right)=1+\left(v_{\mathcal{A}}(M), v_{\mathcal{A}}(M)\right)$ and the last summand only depends on $c_{1}$ and $c_{2}$.

\section{Remark 3.8:}

One could actually write down an exact formula for the dimension using the theory of $\mathcal{A}$-Mukai vectors. But as this is a rather messy formula and it does not bring new understandings of these spaces, we will omit this formula here and compute some dimensions in special cases later.

\section{Corollary 3.9:}

All connected components of the moduli space $M_{\mathcal{A} / \mathbb{P}^{2} ; c_{1}, c_{2}}$ have the same dimension.

\section{Proof:}

By the previous proposition, the dimension of the moudli space at a point depends only on the Chern classes of the bundle which represents that point. But all bundles have the same Chern classes, so this dimension is the same everywhere. 


\subsection{Punctual Quot-Schemes}

\section{Definition 3.10:}

Assume $\mathcal{A}$ is a terminal order on a smooth projective surface $X$ and $M$ is a torsion-free $\mathcal{A}$-module of rank one. Furthermore we choose a point $p \in X$ in the Azumaya locus of $\mathcal{A}$. Then we call the moduli scheme classifying quotients $M \rightarrow T$, where $T$ is a coherent $\mathcal{A}$-module of finite length $l$ supported at $p$, the punctual $\mathcal{A}$-Quot-scheme at $p$ and denote it by:

$$
\operatorname{Quot}_{\mathcal{A}}(M, l, p) \text {. }
$$

\section{Remark 3.11:}

The punctual Quot-scheme depends only on the complete local ring $\hat{\mathcal{O}}_{X, p}$ at $p$. Over the complete local ring the stalk $\hat{\mathcal{A}}_{p}$ is isomorphic to a matrix algebra since $\mathcal{A}$ is unramified at $p$. So we may use Morita equivalence in local computations. For the existence of Quot-schemes, see for example [HL10, Theorem 2.2.4]

\section{Lemma 3.12:}

$\operatorname{Quot}_{\mathcal{A}}(M, l, p)$ is a projective scheme over $k$.

\section{Proof:}

Every $\mathcal{A}$-quotient of $M$ is also a $\mathcal{O}_{X}$-quotient of $M$. If $\operatorname{rank}(\mathcal{A})=r^{2}$ we see, using Morita equivalence, that we have $l_{\mathcal{O}_{X}}(T)=r l_{\mathcal{A}}(T)$.

This shows that $\operatorname{Quot}_{\mathcal{A}}(M, l, p)$ is a subscheme of the projective scheme $Q u o t(M, r l, p)$ classifying $\mathcal{O}_{X}$-quotients of length $r l$ supported at $p$. But $\operatorname{Quot}_{\mathcal{A}}(M, l, p)$ is even a closed subscheme.

To see this, we note that every point $M \rightarrow T$ in $\operatorname{Quot}(M, r l, p)$ defines an exact sequence

$$
0 \longrightarrow N \longrightarrow M \longrightarrow 0 \text {. }
$$

The condition for this point to be in $\operatorname{Quot}_{\mathcal{A}}(M, l, p)$ is that $N$ is an $\mathcal{A}$-submodule of $M$. But this is equivalent to the vanishing of the map $\mathcal{A} \otimes_{\mathcal{O}_{X}} N \rightarrow T$. This map is more explicitly given as the composition of the following maps:

1. $\mathcal{A} \otimes_{\mathcal{O}_{X}} N \hookrightarrow \mathcal{A} \otimes_{\mathcal{O}_{X}} M$ using the exact sequence and the flatness of $\mathcal{A}$;

2. $\mathcal{A} \otimes_{\mathcal{O}_{X}} M \rightarrow M$ using the $\mathcal{A}$-module structure of $M$;

3. $M \rightarrow T$ which is the given surjection.

As a closed subscheme of a projective scheme, $\operatorname{Quot}_{\mathcal{A}}(M, l, p)$ is itself projective over $k$.

\section{Remark 3.13:}

In the same manner one can see that the Flag-scheme $\operatorname{Flag}_{\mathcal{A}}\left(M, l_{1}, l_{2}, p\right)$, classifying iterated quotients $M \rightarrow T_{1} \rightarrow T_{2}$ of length $l_{i}$ supported at $p$, is a projective scheme.

Sending an iterated quotient $M \rightarrow T_{1} \rightarrow T_{2}$ to $M \rightarrow T_{1}$ and $M \rightarrow T_{2}$ defines two morphisms $\pi_{i}: \operatorname{Flag}_{\mathcal{A}}\left(M, l_{1}, l_{2}, p\right) \rightarrow \operatorname{Quot}_{\mathcal{A}}\left(M, l_{i}, p\right)$.

We want to show that the punctual Quot-scheme is connected, to do so, we need the following lemma: 
Lemma 3.14 ([Liu02, Chapter 3.3 Excercise 3.12]):

Assume $f: X \rightarrow Y$ is a surjective proper morphism of schemes. If $Y$ and all fibers $f^{-1}(y)$ are connected, then $X$ is connected.

\section{Proof:}

We note that since $f$ is proper, it is especially a closed morphism, hence it maps closed subsets to closed subsets.

If $X$ were not connected, there would be two non-empty closed subsets $U, V \subset X$ satisfying:

$$
U \cap V=\emptyset \text { and } U \cup V=X .
$$

Since $f$ is surjective we have $Y=f(U \cup V)=f(U) \cup f(V)$. This shows that $Y$ is the union of two non-empty closed subsets, since $f$ is closed.

As $Y$ is connected by assumption, we must have $f(U) \cap f(V) \neq \emptyset$. So we pick $y \in f(U) \cap f(V)$ and let $F$ be the fiber $f^{-1}(y)$ over $y$.

But then $F$ decomposes as $F=(F \cap U) \cup(F \cap V)$. Here $F \cap U$ and $F \cap V$ are two disjoint nonempty closed subsets of $F$. This contradicts the connectedness of $F$. So $X$ must be connected.

Theorem 3.15:

If $M$ is locally projective at $p$, then the punctual Quot-scheme Quot $_{\mathcal{A}}(M, l, p)$ is connected for any $l \geq 1$.

\section{Proof:}

We use induction on $l$. The case $l=1$ asks to find all surjections $M \rightarrow T$ with $l_{\mathcal{A}}(T)=1$. Thus $T$ is a simple $\mathcal{A}$-module, so any nontrivial morphism $M \rightarrow T$ is automatically surjective. Now multiplying a morphism with a constant in $k^{\times}$gives the same quotient. We see that all these quotients are classified by $\left(\operatorname{Hom}_{\mathcal{A}}(M, T) \backslash\{0\}\right) / k^{\times}$. But this a projective space $\mathbb{P}^{n}$ for some $n \geq 1$ and $\mathbb{P}^{n}$ is connected for $n \geq 1$.

So assume $\operatorname{Quot}_{\mathcal{A}}(M, l, p)$ is connected.

We have the two morphisms $\pi_{1}$ and $\pi_{2}$, which give the following diagram:

$$
\begin{aligned}
\operatorname{Flag}_{\mathcal{A}}(M, l+1, l, p) \stackrel{\pi_{1}}{\longrightarrow} \operatorname{Quot}_{\mathcal{A}}(M, l+1, p) \\
\pi_{2} \downarrow \\
\operatorname{Quot}_{\mathcal{A}}(M, l, p)
\end{aligned}
$$

First we will show that $\pi_{1}$ and $\pi_{2}$ are surjective.

For a given point $\left(M \rightarrow T_{1}\right) \in \operatorname{Quot}_{\mathcal{A}}(M, l+1, p)$ we pick a nontrivial element from $\operatorname{Hom}_{\mathcal{A}}\left(S, T_{1}\right)$, where $l_{\mathcal{A}}(S)=1$ and $S$ is supported at $p$. Such an element exitst as $\operatorname{Hom}_{\mathcal{A}}\left(S, T_{1}\right) \neq\{0\}$. This can be seen using Morita equivalence at $p$ and [EL99, Lemma 2].

Since $S$ is a simple $\mathcal{A}$-module, any such nontrivial map must be injective, so we get an exact sequence

$$
0 \longrightarrow S \longrightarrow T_{1} \longrightarrow T_{2} \longrightarrow 0
$$

with $l_{\mathcal{A}}\left(T_{2}\right)=l$ so that $M \rightarrow T_{1} \rightarrow T_{2}$ maps to $M \rightarrow T_{1}$ under $\pi_{1}$, implying that $\pi_{1}$ is surjective. Given $\left(M \rightarrow T_{2}\right) \in \operatorname{Quot}_{\mathcal{A}}(M, l, p)$, let $N$ be the kernel of this surjection.

Using Morita equivalence at $p$ and [EL99, Lemma 2] again, we see that $\operatorname{Hom}_{\mathcal{A}}(N, S) \neq\{0\}$. 
We choose a nontrivial element from $\operatorname{Hom}_{\mathcal{A}}(N, S)$. This element defines the following pushout diagram:

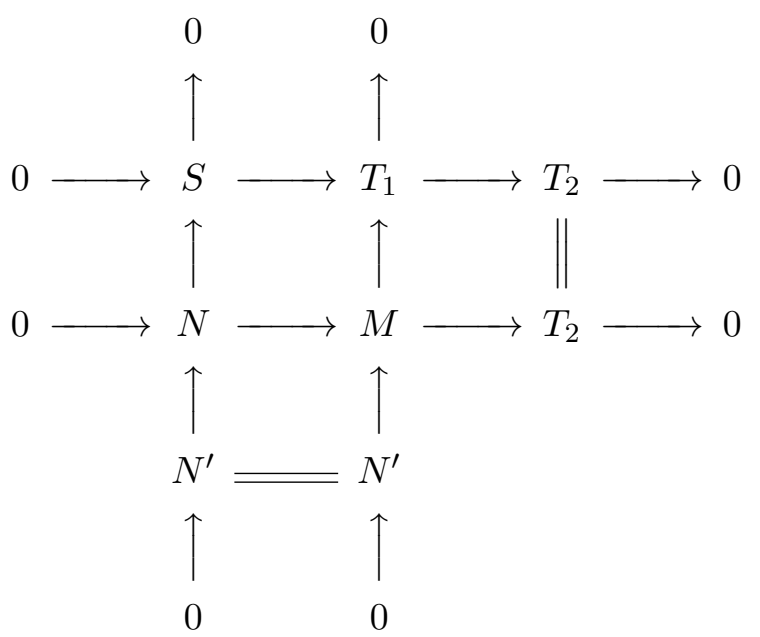

This pushout defines an iterated quotient $\left(M \rightarrow T_{1} \rightarrow T_{2}\right) \in \operatorname{Flag}_{\mathcal{A}}(M, l+1, l, p)$ which maps to $\left(M \rightarrow T_{2}\right)$ under $\pi_{2}$, thus $\pi_{2}$ is also surjective.

This furthermore shows that all possible flags over $M \rightarrow T_{2}$ are parametrized by the projective space $\mathbb{P}\left(\operatorname{Hom}_{\mathcal{A}}(N, S)\right)$. Hence the fiber of $\pi_{2}$ over $\left(M \rightarrow T_{2}\right)$ is connected.

As the Flag-scheme is projective, the morphism $\pi_{2}$ is projective, so it is especially proper. This shows that $\pi_{2}: \operatorname{Flag}_{\mathcal{A}}(M, l+1, l, p) \rightarrow \operatorname{Quot}_{\mathcal{A}}(M, l, p)$ satisfies all conditions of lemma (3.14). We see that $\operatorname{Flag}_{\mathcal{A}}(M, l+1, l, p)$ is connected.

But as $\pi_{1}$ is surjective, we conclude that $\operatorname{Quot}_{\mathcal{A}}(M, l+1, p)$ is connected too.

\section{Remark 3.16:}

Given a torsion-free $\mathcal{A}$-module of rank one $M$ which is locally projective at $p$, we have a map $\operatorname{Quot}_{\mathcal{A}}(M, l, p) \rightarrow M_{\mathcal{A}, X}$ by sending a quotient to its kernel. Since the punctual Quot-scheme is connected, the image of this map is contained in one connected component of the moduli space.

\subsection{Deformations}

Given a terminal del Pezzo order $\mathcal{A}$ on $\mathbb{P}^{2}$ and a torsion-free $\mathcal{A}$-module $M$ of rank one. Then there are two possibilities: either $M$ is even a locally projective $\mathcal{A}$-module, or $M$ is just torsion-free. This fact is captured by the canonical exact sequence

$$
0 \longrightarrow M \stackrel{\iota}{\longrightarrow} M^{* *} \longrightarrow T \longrightarrow 0 .
$$

Since $M$ is torsion-free, it is in fact free in codimension one so that $\iota$ is an isomorphism in codimension one. This implies that $T$ is supported at finitely many points and is therefore an Artinian sheaf.

We denote $\operatorname{supp}(T)$ by $\operatorname{sing}(M)$. The points in $\operatorname{sing}(M)$ are exactly the points in $\mathbb{P}^{2}$ at which $M$ is not locally projective.

The length $n(M):=l_{\mathcal{A}}\left(M^{* *} / M\right)=l_{\mathcal{A}}(T)$ of the Artinian sheaf $T$ is a measure of deviation of $M$ from being locally projective.

The idea is now to use deformation theory to find deformations $N$ of $M$ such that $n(N)<n(M)$. We start with the definition of deformations. 


\section{Definition 3.17:}

$A$ deformation $\mathcal{M}$ of $M$ over a base scheme $B$ is a family of torsion-free $\mathcal{A}$-modules over $B$ such that $\mathcal{M}_{t_{0}}=M$ for some $t_{0} \in B$. For a general $t \in B$ we call the $\mathcal{A}$-module $N:=\mathcal{M}_{t}$ also a deformation of $M$.

\section{Remark 3.18:}

From now, when we talk about the base scheme $B$, we will always mean the pointed space $\left(B, t_{0}\right)$.

\section{Example 3.19:}

It is well known that isomorphism classes of deformations of $M$ as a sheaf of $\mathcal{O}_{X}$-modules over $B=\operatorname{Spec}(R)$ with $R:=k[\epsilon] /\left(\epsilon^{2}\right)$ are classified by $\operatorname{Ext}_{\mathcal{O}_{X}}^{1}(M, M)$.

If we are interested in deformations as a sheaf of $\mathcal{A}$-modules, then these are classified by $\operatorname{Ext}_{\mathcal{A}}^{1}(M, M)$, see [HS05, Lemma 3.1].

Since $B$ comes equipped with a distinguished point $t_{0}$ we can look at the Zariski tangent space $T_{t_{0}} B$ of $B$ at $t_{0}$.

Picking a tangent vector $v \in T_{t_{0}} B$ is the same as giving a morphism $v: \operatorname{Spec}(R) \rightarrow B$ which maps the unique closed point in $\operatorname{Spec}(R)$ to $t_{0}$. This allows us to define a class in $\operatorname{Ext}_{\mathcal{O}_{X}}^{1}(M, M)$ for a given deformation $\mathcal{M}$.

\section{Definition 3.20:}

The Kodaira-Spencer map of a deformation $\mathcal{M}$ is a map

$$
\theta_{\mathcal{M}}: T_{t_{0}} B \rightarrow \operatorname{Ext}_{\mathcal{O}_{X}}^{1}(M, M)
$$

defined the following way: choose a Zariski tangent vector $v \in T_{t_{0}} B$ and identify it with a map $v: \operatorname{Spec}(R) \rightarrow B$. Then we can pullback $\mathcal{M}$ along the map $f:=\left(i d_{X} \times v\right): X \times \operatorname{Spec}(R) \rightarrow X \times B$ and get a deformation $f^{*} \mathcal{M}$ of $M$ over the base $\operatorname{Spec}(R)$. Its isomorphism class defines an element in $\operatorname{Ext}_{\mathcal{O}_{X}}^{1}(M, M)$.

Now we want to find deformations $N$ of $M$ which are somehow better than $M$ in the sense that they are closer to being locally projective than $M$, meaning $n(N)<n(M)$. To find such deformations we will use a result of Artamkin in [Art91], which we will sketch now, as some of the ideas are used latter.

Assume $X$ is a smooth projective surface and $F$ is a coherent torsion-free simple $\mathcal{O}_{X}$-module, that is $\operatorname{End}_{\mathcal{O}_{X}}(F)=k$.

We have the standard short exact sequence:

$$
0 \longrightarrow F \longrightarrow F^{* *} \longrightarrow S \longrightarrow 0 .
$$

Applying $\mathcal{H o m}_{\mathcal{O}_{X}}\left(-, \mathcal{O}_{X}\right)$ shows that

$$
\mathcal{E} x t_{\mathcal{O}_{X}}^{1}\left(F, \mathcal{O}_{X}\right) \cong \mathcal{E} x t_{\mathcal{O}_{X}}^{2}\left(S, \mathcal{O}_{X}\right) .
$$

This is due to the fact that $\mathcal{E}_{x} t_{\mathcal{O}_{X}}^{i}\left(F^{* *}, \mathcal{O}_{X}\right)=0$ for $i=1,2$ as $F^{* *}$ locally free.

Now pick $\xi \in \operatorname{Ext}_{\mathcal{O}_{X}}^{1}(F, F)$. This element defines an extension

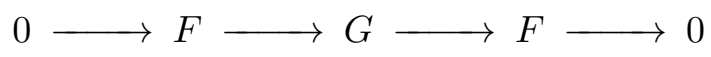


which in turn gives us the dual exact sequence

$$
0 \longrightarrow F^{*} \longrightarrow G^{*} \longrightarrow F^{*} \stackrel{j(\xi)}{\longrightarrow} \mathcal{E} x t_{\mathcal{O}_{X}}^{1}\left(F, \mathcal{O}_{X}\right),
$$

by again applying $\mathcal{H o m}_{\mathcal{O}_{X}}\left(-, \mathcal{O}_{X}\right)$.

That is $j(\xi) \in \operatorname{Hom}\left(F^{*}, \mathcal{E} x t_{\mathcal{O}_{X}}^{1}\left(F, \mathcal{O}_{X}\right)\right)$. Using (10) shows that $j(\xi) \in \operatorname{Hom}\left(F^{*}, \mathcal{E} t_{\mathcal{O}_{X}}^{2}\left(S, \mathcal{O}_{X}\right)\right)$. As $F$ is coherent, the sheaf $F^{*}$ must be reflexive. Because $X$ is a smooth projective surface this implies that $F^{*}$ is locally free. By [Har77, Proposition III.6.7] we get isomorphisms:

$$
\operatorname{Hom}\left(F^{*}, \mathcal{E} x t_{\mathcal{O}_{X}}^{2}\left(S, \mathcal{O}_{X}\right)\right) \cong \operatorname{Hom}\left(\mathcal{O}_{X}, \mathcal{E} x t_{\mathcal{O}_{X}}^{2}\left(S, \mathcal{O}_{X}\right) \otimes F^{* *}\right) \cong \operatorname{Hom}\left(\mathcal{O}_{X}, \mathcal{E} x t_{\mathcal{O}_{X}}^{2}\left(S, F^{* *}\right)\right) .
$$

We conclude that $j(\xi) \in H^{0}\left(X, \mathcal{E} x t_{\mathcal{O}_{x}}^{2}\left(S, F^{* *}\right)\right)$.

As $S$ is Artinian we see that in fact we have $j(\xi) \in \operatorname{Ext}_{\mathcal{O}_{X}}^{2}\left(S, F^{* *}\right)$. Thus we have constructed a map:

$$
j: \operatorname{Ext}_{\mathcal{O}_{X}}^{1}(F, F) \rightarrow \operatorname{Ext}_{\mathcal{O}_{X}}^{2}(S, F) .
$$

\section{Remark 3.21:}

Since we have a decomposition $S=\bigoplus_{i=0}^{n} S_{p_{i}}$, we also have a decomposition $j(\xi)=\bigoplus_{i=0}^{n} j_{p_{i}}(\xi)$.

Thus there is a map:

$$
j_{p_{i}}: \operatorname{Ext}_{\mathcal{O}_{X}}^{1}(F, F) \rightarrow \operatorname{Ext}_{\mathcal{O}_{X}}^{2}\left(S_{p_{i}}, F^{* *}\right) .
$$

The last Ext-group only depends on $p_{i}$, that is:

$$
\operatorname{Ext}_{\mathcal{O}_{X}}^{2}\left(S_{p_{i}}, F^{* *}\right)=H^{0}\left(X, \mathcal{E} x t_{\mathcal{O}_{X}}^{2}\left(S_{p_{i}}, F^{* *}\right)\right)=\operatorname{Ext}_{\mathcal{O}_{X, p_{i}}}^{2}\left(S_{p_{i}}, F_{p_{i}}^{* *}\right) .
$$

So we can also work with the exact sequence

$$
0 \longrightarrow F \longrightarrow F_{i} \longrightarrow S_{p_{i}} \longrightarrow 0,
$$

where $F_{i}$ is given as the kernel of the surjection $F^{* *} \rightarrow \bigoplus_{i=0}^{n} S_{p_{i}} \rightarrow S_{p_{i}}$. We see that $F_{i}$ agrees with $F$ away from $p_{i}$ and agrees with $F^{* *}$ in a neighbourhood of $p_{i}$.

\section{Remark 3.22:}

The map $j: \operatorname{Ext}_{\mathcal{O}_{X}}^{1}(F, F) \rightarrow \operatorname{Ext}_{\mathcal{O}_{X}}^{2}\left(S, F^{* *}\right)$ can also be constructed by another way: we start with the exact sequence

$$
0 \longrightarrow F \stackrel{\iota}{\longrightarrow} F^{* *} \longrightarrow S \longrightarrow 0 .
$$

Then we have induced long exact sequences:

$$
\ldots \longrightarrow \operatorname{Ext}_{\mathcal{O}_{X}}^{1}(F, F) \stackrel{\delta}{\longrightarrow} \operatorname{Ext}_{\mathcal{O}_{X}}^{2}(S, F) \longrightarrow \operatorname{Ext}_{\mathcal{O}_{X}}^{2}\left(F^{* *}, F\right) \longrightarrow \ldots
$$

using $\operatorname{Hom}_{\mathcal{O}_{X}}(-, F)$. Here $\delta$ is the connecting homomorphism.

By applying $\operatorname{Hom}_{\mathcal{O}_{X}}(S,-)$ we get:

$$
\ldots \longrightarrow \operatorname{Ext}_{\mathcal{O}_{X}}^{2}(S, F) \stackrel{\iota_{*}}{\longrightarrow} \operatorname{Ext}_{\mathcal{O}_{X}}^{2}\left(S, F^{* *}\right) \longrightarrow \operatorname{Ext}_{\mathcal{O}_{X}}^{2}(S, S) \longrightarrow 0 .
$$

Then we have $j=\iota_{*} \circ \delta$, see [Art91, Lemma 6.2]. 


\section{Remark 3.23:}

There are canonical surjective trace maps:

$$
t r^{i}: \operatorname{Ext}_{\mathcal{O}_{X}}^{i}(F, F) \rightarrow H^{i}\left(X, \mathcal{O}_{X}\right) .
$$

If one defines $a d^{i}(F)=\operatorname{ker}\left(t r^{i}\right)$, then $a d^{1}(F)$ corresponds to deformations of $F$ with constant determinant.

For example if $X=\mathbb{P}^{2}$ then we have $a d^{1}(F)=\operatorname{Ext}_{\mathcal{O}_{X}}^{1}(F, F)$.

\section{Definition 3.24:}

Assume $\mathcal{F}$ is a deformation of $F$ over a base $B$, then we define the following function on $B$ :

$$
n: B \rightarrow \mathbb{Z}, t \mapsto n(t)=n\left(\mathcal{F}_{t}\right)
$$

Furthermore we define a natural number by:

$$
n^{\mathcal{F}}=\min _{t \in B} n(t)
$$

\section{Remark 3.25:}

Similary one has functions $n_{p_{i}}$ and numbers $n_{p_{i}}^{\mathcal{F}}$ for the points in $\operatorname{sing}(F)$, so that we have for example:

$$
n_{p_{i}}\left(t_{0}\right)=l_{\mathcal{O}_{X}}\left(S_{p_{i}}\right)
$$

Definition 3.26 ([Art91, Chapter 1]):

A point $p_{i}$ in sing $(F)$ is said to be cancellable in the deformation $\mathcal{F}$ if $n_{p_{i}}^{\mathcal{F}}<n_{p_{i}}\left(t_{0}\right)$. This means that in the deformation $\mathcal{F}$ the order of the singularity $p_{i}$ decreases.

We have the following aforementioned result of Artamkin:

Theorem 3.27 ([Art91, Corollary 1.3]):

If $\xi \in \operatorname{Ext}_{\mathcal{O}_{X}}^{1}(F, F)$ is the Kodaira-Spencer class of a deformation $\mathcal{F}$ of $F$ over a one dimensional base $B$ with the property $j_{p_{i}}(\xi) \neq 0$, then $p_{i}$ is cancellable in $\mathcal{F}$.

In [HS05] the authors prove the following theorem about deformations:

Theorem 3.28 ([HS05, Theorem 3.6.(iii)]):

Assume $X$ is a smooth $K 3$ or abelian surface and $\mathcal{A}$ is an Azumaya algebra on $X$. If $M$ is a torsion-free $\mathcal{A}$-module of rank one with Chern classes $c_{1}$ and $c_{2}$, then there is a deformation $N$ of $M$ such that $N$ is a locally projective $\mathcal{A}$-module with the same Chern classes as $M$.

Unfortunately we cannot prove such a strong result in the ramified case.

Using the fact that we have a decomposition $\mathbb{P}^{2}=D \cup\left(\mathbb{P}^{2} \backslash D\right)$, where $D$ is the ramification locus of $\mathcal{A}$, we get an induced decomposition $\operatorname{sing}(M)=H^{A z} \cup H^{r}$. Here we have:

$$
H^{A z}=\operatorname{sing}(M) \cap\left(\mathbb{P}^{2} \backslash D\right) \text { and } H^{r}=\operatorname{sing}(M) \cap D .
$$

So for a point $p \in \operatorname{sing}(M)$ there are two possibilities: either $\mathcal{A}$ is an Azumaya algebra at $p$ or $\mathcal{A}$ is ramified at $p$. We will consider these cases separately. 


\section{Theorem 3.29:}

Assume $\mathcal{A}$ is a terminal del Pezzo order on $\mathbb{P}^{2}$. If $M$ is a torsion-free $\mathcal{A}$-module of rank one, then there is a deformation $N$ of $M$ such that $\operatorname{sing}(N) \subset D$ and $N$ has the same Chern classes as $M$.

\section{Proof:}

Using remark (3.21) it is enough to show the following: given a torsion-free $\mathcal{A}$-module $M$ of rank one, which is not locally projective at some point $p \in \operatorname{sing}(M) \cap\left(\mathbb{P}^{2} \backslash D\right)$, then there is a deformation $N$ of $M$ which is locally projective at $p$ and has the same Chern classes as $M$. This implies $\operatorname{sing}(N)=\operatorname{sing}(M) \backslash\{p\}$.

Because if we have this result, we can simply apply it to the finitely many points belonging to $\operatorname{sing}(M)$ one at a time. Since a deformation of a deformation is still a deformation, we finally get a deformation $N$ of $M$ with the same Chern classes as $M$, such that $\operatorname{sing}(N) \cap\left(\mathbb{P}^{2} \backslash D\right)=\emptyset$ implying $\operatorname{sing}(N) \subset D$.

To prove the mentioned result, we start by forming the bidual of $M$ which gives the quotient:

$$
M^{* *} / M=T=\bigoplus_{p \in \operatorname{sing}(M)} T_{p}
$$

For $p \in \operatorname{sing}(M) \cap\left(\mathbb{P}^{2} \backslash D\right)$ we can look at the quotient $M^{* *} \rightarrow T \rightarrow T_{p}$. Let $M^{A z}$ be its kernel. This shows that we have a short exact sequence:

$$
0 \longrightarrow M \longrightarrow M^{A z} \longrightarrow T_{p} \longrightarrow 0 \text {. }
$$

Here $M^{A z}$ agrees with the bidual $M^{* *}$ at $p$, particularly $M^{A z}$ is locally projective at $p$, and at all other points $M^{A z}$ agrees with $M$. This sequence defines an element in the punctual Quot-scheme $\operatorname{Quot}_{\mathcal{A}}\left(M^{A z}, l, p\right)$ for some $l \geq 1$. By (3.15) this scheme is connected.

We may assume that this sequence is as simple as possible, meaning it is locally at $p$ Morita equivalent to an exact sequence of the form:

$$
0 \longrightarrow I \oplus \mathcal{O}_{p}^{r-1} \longrightarrow \mathcal{O}_{p}^{r} \longrightarrow \mathcal{O}_{p} / I \longrightarrow 0 .
$$

Here $\mathcal{O}_{p}=\widehat{\mathcal{O}}_{X, p}$ is the complete local ring at $\mathrm{p}$ and $I \subset \mathcal{O}_{p}$ is an ideal of colength $l$.

We can do this because the Quot-scheme is connected, that is all other possible kernels $\tilde{M}$ of $M^{A z} \rightarrow T_{p}$ lie in the same connected component of the moduli space as $M$, see remark (3.16). Since the moduli space is smooth we can choose a smooth connected curve which connects $M$ and $\tilde{M}$. This curve defines a deformation of $M$ (to $\tilde{M}$ ), which allows us to work with $\tilde{M}$ instead of $M$.

Now we modify the argument given in [HS05], which uses the result of Artamkin. We consider the diagram, resulting from (11):

$$
\begin{aligned}
\operatorname{Ext}_{\mathcal{A}}^{1}(M, M) \stackrel{\delta}{ } & \operatorname{Ext}_{\mathcal{A}}^{2}\left(T_{p}, M\right) \longrightarrow \operatorname{Ext}_{\mathcal{A}}^{2}\left(M^{A z}, M\right) \\
& \downarrow^{\iota_{*}} \\
& \operatorname{Ext}_{\mathcal{A}}^{2}\left(T_{p}, M^{A z}\right)
\end{aligned}
$$

Since $c_{1}(M)=c_{1}\left(M^{A z}\right)$ we have $\operatorname{Ext}_{\mathcal{A}}^{2}\left(M^{A z}, M\right)=0$ by (3.4). This implies that the connecting homomorphism $\delta$ is surjective.

Furthermore the map $\iota_{*}$ is nontrivial. To see this we assume the contrary: $\iota_{*}=0$. Then the long exact sequence asssociated to $\operatorname{Hom}_{\mathcal{A}}\left(T_{p},-\right)$ shows that there must be an isomorphism: 


$$
\operatorname{Ext}_{\mathcal{A}}^{2}\left(T_{p}, M^{A z}\right) \cong \operatorname{Ext}_{\mathcal{A}}^{2}\left(T_{p}, T_{p}\right) .
$$

Using Serre duality, $\omega_{\mathcal{A}, p}=\mathcal{A}_{p}$ (since $\mathcal{A}$ is unramified at $p$ ) and Morita equivalence, this isomorphism corresponds to the isomorphism:

$$
\operatorname{Hom}_{\mathcal{O}_{p}}\left(\mathcal{O}_{p} / I, \mathcal{O}_{p} / I\right) \cong \operatorname{Hom}_{\mathcal{O}_{p}}\left(\mathcal{O}_{p}^{r}, \mathcal{O}_{p} / I\right) .
$$

But we certainly have:

$$
\operatorname{Hom}_{\mathcal{O}_{p}}\left(\mathcal{O}_{p} / I, \mathcal{O}_{p} / I\right) \cong \mathcal{O}_{p} / I \text { and } H_{o m} \mathcal{O}_{p}\left(\mathcal{O}_{p}^{r}, \mathcal{O}_{p} / I\right)=\left(\mathcal{O}_{p} / I\right)^{r}
$$

Here $\mathcal{O}_{p} / I$ has length $l$ by assumption. But $\left(\mathcal{O}_{p} / I\right)^{r}$ has length $r l$. Since $\mathcal{A}$ is nontrivial, we have $r \geq 2$. This shows that there is no such isomorphism as $l \neq r l$, hence $\iota_{*}$ must be nontrivial. We can thus find $\xi \in \operatorname{Ext}_{\mathcal{A}}^{1}(M, M)$ whose image in $\operatorname{Ext}_{\mathcal{A}}^{2}\left(T_{p}, M^{A z}\right)$ is non-zero: by the previous argument we can pick $\gamma \in \operatorname{Ext}_{\mathcal{A}}^{2}\left(T_{p}, M\right)$ with $\iota_{*}(\gamma) \neq 0$. As $\delta$ is surjective, we can find a class $\xi \in \operatorname{Ext}_{\mathcal{A}}^{1}(M, M)$ with $\delta(\xi)=\gamma$. This implies $\iota_{*}(\delta(\xi)) \neq 0$ in $\operatorname{Ext}_{\mathcal{A}}^{2}\left(T_{p}, M^{A z}\right)$.

Now there is a deformation $\mathcal{M}$ of $M$ over a smooth connected curve $B$ whose Kodaira-Spencer class is $\xi$. This can be seen in the following way: we have an isomorphism for the tangent space at $[M]$ :

$$
T_{[M]} M_{\mathcal{A} / \mathbb{P}^{2} ; c_{1}, c_{2}} \cong \operatorname{Ext}_{\mathcal{A}}^{1}(M, M) .
$$

This shows that $\xi$ correspondends to a morphism $v: \operatorname{Spec}\left(k[\epsilon] /\left(\epsilon^{2}\right)\right) \rightarrow M_{\mathcal{A} / \mathbb{P}^{2} ; c_{1}, c_{2}}$ such that the unique closed point gets mapped to $[M]$. The smoothness of the moduli space implies that we can find a smooth connected curve $B$ with a distinguished point $t_{0} \in B$ and an embedding $j: B \hookrightarrow M_{\mathcal{A} / \mathbb{P}^{2} ; c_{1}, c_{2}}$ such that:

- the embedding $j$ maps $t_{0}$ to $[M]$;

- $j(B)$ has tangent vector $v$ at $[M]$.

Then $B$ and $j$ define a deformation $\mathcal{M}$ with the desired properties.

For a generic $t \in B$, that is especially $t \neq t_{0}$, let $N=\mathcal{M}_{t}$ be the fiber of $\mathcal{M}$ over $t$. Forming the bidual we get an exact sequence:

$$
0 \longrightarrow N \longrightarrow N^{* *} \longrightarrow S \longrightarrow 0 \text {. }
$$

By construction, $\mathrm{S}$ is also supported only at $p$. Using Lemma (1.62) shows that the forgetful map:

$$
\operatorname{Ext}_{\mathcal{A}}^{2}\left(T_{p}, M^{A z}\right) \rightarrow \operatorname{Ext}_{\mathcal{O}_{\mathbb{P} 2}}^{2}\left(T_{p}, M^{A z}\right)
$$

is injective.

This implies that the Kodaira-Spencer class of $\mathcal{M}$, as an element in $\operatorname{Ext}_{\mathcal{O}_{\mathbb{P} 2}}^{1}(M, M)$, has nonzero image in $\operatorname{Ext}_{\mathcal{O}_{\mathbb{P} 2}}^{2}\left(T_{p}, M^{A z}\right)$. By the theorem of Artamkin (3.27) this implies that $N$ is less singular at $p$. That is: the length of $S_{p}$ as an $\mathcal{O}_{\mathbb{P}^{2}, p^{-}}$module is strictly less than $l r$. But $S$ is an $\mathcal{A}$-module, so the length at $p$ must be divisible by $r$ since $\mathcal{A}$ is an Azumaya algebra of rank $r^{2}$ at this point, implying it has $\mathcal{O}_{\mathbb{P}^{2}}$-length less or equal to $(l-1) r$ so $l_{\mathcal{A}}\left(T_{p}\right) \leq l-1$.

Repeating this process finitely many times we end up with a quotient whose length is strictly less then $r$ but also divisible by $r$, implying it must be zero. That is $N$ is locally projective at p. It remains to find the Chern classes of $N$. But we have: 


$$
c_{i}(N)=c_{i}\left(\mathcal{M}_{t}\right)=c_{i}\left(\mathcal{M}_{t_{0}}\right)=c_{i}(M),
$$

due to the fact that the Chern classes, as elements of $H^{2 i}(X, \mathbb{Q})$, are locally constant in a flat family. Since the base $B$ is connected they must be constant.

\section{Theorem 3.30:}

Assume $\mathcal{A}$ is a terminal del Pezzo order on $\mathbb{P}^{2}$ and $M$ is a torsion-free $\mathcal{A}$-module of rank one. If $\operatorname{sing}(M) \subset D$ and every $p \in \operatorname{sing}(M)$ is a smooth point of $D$ with $l_{\mathcal{A}_{p}}\left(M_{p}^{* *} / M_{p}\right)=1$, then there is deformation $N$ of $M$ such that $N$ is locally projective and has the same Chern classes as $M$.

\section{Proof:}

Using remark (3.21) again and the same argument as in the previous theorem, we see that it is enough to prove this for the case $\operatorname{sing}(M)=\{p\}$.

We have the canonical exact sequence

$$
0 \longrightarrow M \stackrel{\iota}{\longrightarrow} M^{* *} \longrightarrow T \longrightarrow 0 \text {. }
$$

This sequence induces the following diagram

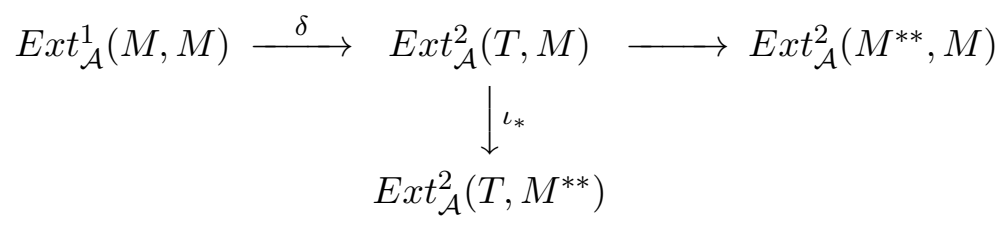

Since $c_{1}(M)=c_{1}\left(M^{* *}\right)$ we have $\operatorname{Ext}_{\mathcal{A}}^{2}\left(M^{* *}, M\right)=0$ by (3.4). This shows that the connecting homomorphism $\delta$ is surjective.

We will show that in this case $\iota_{*}$ is nontrivial. Using the following sequence:

$$
\operatorname{Ext}_{\mathcal{A}}^{2}(T, M) \stackrel{\iota_{*}}{\longrightarrow} \operatorname{Ext}_{\mathcal{A}}^{2}\left(T, M^{* *}\right) \longrightarrow \operatorname{Ext}_{\mathcal{A}}^{2}(T, T) \longrightarrow 0,
$$

it is enough to see that $\operatorname{Ext}_{\mathcal{A}}^{2}(T, T)=0$.

Since both questions are local at $p$ we may work over the complete local ring $R=\hat{\mathcal{O}}_{X, p}$ at $p$. There $A=\hat{\mathcal{A}}_{p}$ is isomorphic to $M_{f}(B)$ for some $f \geq 1$, see (1.9). We will distinguish the cases $f=1$ and $f>1$. We start with the case $f=1$.

By (2.16) we have $\chi_{\mathcal{A}}(T, T)=0$, since $T$ is an Artinian $\mathcal{A}$-module.

As $T$ is a simple Artinian $\mathcal{A}$-module and $k$ is algebraically closed, we must have $\operatorname{Hom}_{\mathcal{A}}(T, T)=k$ by Schur's lemma. To see that $\operatorname{Ext}_{\mathcal{A}}^{2}(T, T)=0$ it is thus enough to show that $\operatorname{Ext}_{\mathcal{A}}^{1}(T, T)=k$. To do this we begin once more with:

$$
0 \longrightarrow M \longrightarrow M^{* *} \longrightarrow T \longrightarrow 0,
$$

and look at the long sequence induced by $\operatorname{Hom}_{\mathcal{A}}(-, T)$ :

$$
\begin{aligned}
0 \longrightarrow \operatorname{Hom}_{\mathcal{A}}(T, T) & \longrightarrow \operatorname{Hom}_{\mathcal{A}}\left(M^{* *}, T\right) \longrightarrow \operatorname{Hom}_{\mathcal{A}}(M, T) \\
\longrightarrow \operatorname{Ext}_{\mathcal{A}}^{1}(T, T) \longrightarrow \operatorname{Ext}_{\mathcal{A}}^{1}\left(M^{* *}, T\right) \longrightarrow & \ldots
\end{aligned}
$$

Since $M^{* *}$ is locally projective and $T$ is Artinian, we have:

$$
\operatorname{Ext}_{\mathcal{A}}^{1}\left(M^{* *}, T\right)=\operatorname{Ext}_{\mathcal{A}_{p}}^{1}\left(M_{p}^{* *}, T_{p}\right)=0 .
$$


Using (1.37) we see that:

$$
\operatorname{Hom}_{\mathcal{A}}\left(M^{* *}, T\right)=\operatorname{Hom}_{\mathcal{A}_{p}}\left(M_{p}^{* *}, T_{p}\right) \cong \operatorname{Hom}_{\mathcal{A}_{p}}\left(\mathcal{A}_{p}, T_{p}\right)=T_{p}=k,
$$

where the last equality uses the complete local structure of $T$, given by (16).

This shows that there is an isomorphism:

$$
H o m_{\mathcal{A}}(T, T) \cong H o m_{\mathcal{A}}\left(M^{* *}, T\right) .
$$

The long sequence therefore induces an isomorphism:

$$
\operatorname{Hom}_{\mathcal{A}}(M, T) \cong \operatorname{Ext}_{\mathcal{A}}^{1}(T, T) .
$$

Thus we have reduced the problem to show that $\operatorname{Hom}_{\mathcal{A}}(M, T)=k$.

Using (1.9) and the fact that $f=1$, we see that the algebra $A$ is given by:

$$
\left(\begin{array}{cccc}
R & \cdots & \cdots & R \\
u R & R & \ddots & \vdots \\
\vdots & \ddots & \ddots & \vdots \\
u R & \cdots & u R & R
\end{array}\right)
$$

We have an isomorphism $\hat{M}_{p}^{* *} \cong \hat{\mathcal{A}}_{p}$ by $(1.37)$, as $T_{p}$ is simple we can identify $\hat{M}_{p}$ with a maximal left ideal $\mathfrak{m}$ in $A$ :

$$
\left(\begin{array}{cccc}
(u, v) & \cdots & \cdots & R \\
u R & R & \ddots & \vdots \\
\vdots & \ddots & \ddots & \vdots \\
u R & \cdots & u R & R
\end{array}\right)
$$

We remark that there are other maximal left ideals. These are those ideals with exactly one $(u, v)$-term is on the diagonal. Since all the following computations are equivalent for these ideals, we choose without loss of generality the maximal ideal described above.

We then conclude that $T$ is given locally at $p$ by:

$$
\left(\begin{array}{cccc}
k & \cdots & \cdots & 0 \\
0 & 0 & \ddots & \vdots \\
\vdots & \ddots & \ddots & \vdots \\
0 & \cdots & 0 & 0
\end{array}\right)
$$

Now we want to determine $\operatorname{Hom}_{A}(\mathfrak{m}, A / \mathfrak{m})$.

First we further reduce to determine: $\operatorname{Hom}_{A}\left(\mathfrak{m} / \mathfrak{m}^{2}, A / \mathfrak{m}\right):$ if $\phi: \mathfrak{m} \rightarrow A / \mathfrak{m}$ is a morphism, then $\phi\left(\mathfrak{m}^{2}\right)=\mathfrak{m} \cdot \phi(\mathfrak{m})=0$ since $\phi$ is $A$-linear. So $\mathfrak{m}^{2} \subset \operatorname{ker}(\phi)$ and $\phi$ induces a map $\mathfrak{m} / \mathfrak{m}^{2} \rightarrow A / \mathfrak{m}$. From the description given above:

$$
\mathfrak{m}=\left(\begin{array}{cccc}
(u, v) & \cdots & \cdots & R \\
u R & R & \ddots & \vdots \\
\vdots & \ddots & \ddots & \vdots \\
u R & \cdots & u R & R
\end{array}\right)
$$


it follows that $\mathfrak{m}^{2}$ is given by:

$$
\mathfrak{m}^{2}=\left(\begin{array}{cccc}
(u, v)^{2}+(u) & \cdots & \cdots & R \\
u R & R & \ddots & \vdots \\
\vdots & \ddots & \ddots & \vdots \\
u R & \cdots & u R & R
\end{array}\right)
$$

But as ideals in $R$ we have: $(u, v)^{2}+(u)=\left(u^{2}, u v, v^{2}\right)+(u)=\left(u, v^{2}\right)$. We conclude:

$$
\mathfrak{m} / \mathfrak{m}^{2} \cong\left(\begin{array}{cccc}
(u, v) /\left(u, v^{2}\right) & \cdots & \cdots & 0 \\
0 & 0 & \ddots & \vdots \\
\vdots & \ddots & \ddots & \vdots \\
0 & \cdots & 0 & 0
\end{array}\right) \cong\left(\begin{array}{cccc}
k & \cdots & \cdots & 0 \\
0 & 0 & \ddots & \vdots \\
\vdots & \ddots & \ddots & \vdots \\
0 & \cdots & 0 & 0
\end{array}\right)
$$

This shows that $\operatorname{Hom}_{A}(\mathfrak{m}, A / \mathfrak{m})=k$. So $\operatorname{Hom}_{\mathcal{A}}(M, T)=k$, implying $\operatorname{Ext}_{\mathcal{A}}^{1}(T, T)=k$. Putting everything together shows that $\operatorname{Ext}_{\mathcal{A}}^{2}(T, T)=0$.

Thus the sequence (13) degenerates to

$$
\operatorname{Ext}_{\mathcal{A}}^{2}(T, M) \stackrel{\iota_{*}}{\longrightarrow} \operatorname{Ext}_{\mathcal{A}}^{2}\left(T, M^{* *}\right) \longrightarrow 0 .
$$

This means that $\iota_{*}$ is a surjective map between nontrivial spaces, hence $\iota_{*}$ is nontrivial.

Now assume $f>1$, then $A=M_{f}(B)$ is Morita equivalent to the algebra $B$ described in (1.9). The sequence (14) is locally at $p$ Morita equivalent to:

$$
0 \longrightarrow \mathfrak{m} \oplus B^{f-1} \longrightarrow B^{f} \longrightarrow S \longrightarrow 0
$$

where $\mathfrak{m}$ is the maximal left ideal of $B$ described above and $S$ the associated simple $B$-module. Then long exact sequence associted to $\operatorname{Hom}_{B}(-, S)$ is given by:

$$
\begin{gathered}
0 \longrightarrow \operatorname{Hom}_{B}(S, S) \longrightarrow \operatorname{Hom}_{B}\left(B^{f}, S\right) \longrightarrow \operatorname{Hom}_{B}\left(\mathfrak{m} \oplus B^{f-1}, S\right) \\
\left.\longrightarrow \operatorname{Ext}_{B}^{1}(S, S) \longrightarrow B^{f}, S\right) \longrightarrow
\end{gathered}
$$

Again we have $\operatorname{Hom}_{B}(S, S)=k$ by Schur's lemma and $\operatorname{Ext}_{B}^{1}\left(B^{f}, S\right)=0$ since $B^{f}$ is a free $B$-module. Furthermore $\operatorname{Hom}_{B}\left(B^{f}, S\right)=k^{f}$ and by the previous computations we see that $H o m_{B}\left(\mathfrak{m} \oplus B^{f-1}, S\right)=k^{f}$.

As the Euler characteristic of the long sequence must vanish, we conclude that $\operatorname{Ext}_{B}^{1}(S, S)=k$. So by Morita equivalence $\operatorname{Ext}_{\mathcal{A}}^{1}(T, T)=k$ and again $\operatorname{Ext}_{\mathcal{A}}^{2}(T, T)=0$ so that $\iota_{*}$ is also nontrivial in this case.

Knowing this we can argue as in the previous theorem: since $\delta$ is surjective and $\iota_{*}$ is nontrivial we can pick $\xi \in \operatorname{Ext}_{\mathcal{A}}^{1}(M, M)$ with nontrivial image in $\operatorname{Ext}_{\mathcal{A}}^{2}\left(T, M^{* *}\right)$.

There is a deformation $\mathcal{M}$ of $M$ over a smooth connected curve $B$ with Kodaira-Spencer class $\xi$. The fiber $N=\mathcal{M}_{t}$ over a general $t \in B$ must be locally projective at $p$ using the result of Artamkin and the fact that the length as an $\mathcal{O}_{\mathbb{P}^{2}}$-module must be divisible by $f$ in this case. Furthermore the Chern classes of $N$ and $M$ agree for the same reasons as in the previous theorem.

In the following we denote by $M_{\mathcal{A} / X ; c_{1}, c_{2}}^{l p}$ the open subscheme of the moduli space $M_{\mathcal{A} / X ; c_{1}, c_{2}}$ which classifies only locally projective $\mathcal{A}$-modules of rank one. If $\mathcal{A}$ is a maximal order of rank $r^{2}$, then for every ramification curve $C$ with ramification index $e$, the natural number $f:=\frac{r}{e}$ is well defined, see [Art86, Definition 2.13]. 


\section{Corollary 3.31:}

Assume $\mathcal{A}$ is a terminal del Pezzo order on $\mathbb{P}^{2}$ with ramification curves $\left\{C_{i}\right\}$, ramification indices $\left\{e_{i}\right\}$ and associated numbers $\left\{f_{i}\right\}$ for $i=1, \ldots$, l. If $M_{\mathcal{A} / X ; c_{1}, c_{2}}^{l p} \neq \emptyset$ then $M_{\mathcal{A} / X, c_{1}, c_{2}+f_{i}}^{l p} \neq \emptyset$ for every $i=1, \ldots, l$.

\section{Proof:}

It is enough to show this for a fixed ramification curve $C$ with ramificationd index $e$ and associated number $f$.

Since $M_{\mathcal{A} / X ; c_{1}, c_{2}}^{l p} \neq \emptyset$, we can pick a locally projective $\mathcal{A}$-module $M$ of rank one with the desired Chern classes.

We choose a point $p$ on $C$ which is a nonsingular point of the ramification locus. Furthermore we choose a simple Artinian $\mathcal{A}$-module $T$ which is supported at $p$. Using the knowledge of the complete local structure at $p$ we conclude that $l_{\mathcal{O}_{X}}(T)=f l_{\mathcal{A}}(T)=f$ implying $c_{2}(T)=-f$. Using (1.37) and $T \neq 0$, we see that $\operatorname{Hom}_{\mathcal{A}}(M, T) \neq\{0\}$. Thus we get a short exact sequence:

$$
0 \longrightarrow \mathrm{K} \longrightarrow \mathrm{M} \longrightarrow \mathrm{T} \longrightarrow 0,
$$

here $K:=\operatorname{ker}(N \rightarrow S)$.

We compute the Chern classes and see that:

$$
c_{1}(K)=c_{1}(M) \text { and } c_{2}(K)=c_{2}(M)-c_{2}(T)=c_{2}(M)+f .
$$

As $T$ is non-zero the $\mathcal{A}$-module $K$ cannot be locally projective. But by construction $M=K^{* *}$ and $l_{\mathcal{A}}\left(K^{* *} / K\right)=1$ so we can use (3.30) to see that we can deform $K$ into a locally projective $\mathcal{A}$-module $N$ with the same Chern classes, so $N$ defines a point in $M_{\mathcal{A} / X, c_{1}, c_{2}+f}^{l p}$.

\section{Corollary 3.32:}

Assume $\mathcal{A}$ is a terminal del Pezzo order on $\mathbb{P}^{2}$. If $M_{\mathcal{A} / X ; c_{1}, c_{2}}^{l p} \neq \emptyset$ then $M_{\mathcal{A} / X, c_{1}, c_{2}+n f_{i}}^{l p} \neq \emptyset$ for all $i=1, \ldots, l$ and any $n \geq 1$.

\section{Proof:}

Use the previous Corollary $n$-times.

The last corollary shows that if the open subscheme of locally projective $\mathcal{A}$-modules of rank one is nonempty for one pair of Chern classes, then there are infinitely many pairs of Chern classes for which this subscheme is non-empty. Especially we obtain infinitely many non-empty moduli spaces. Since $\mathcal{A}$ is always a locally projective $\mathcal{A}$-module of rank one, we also have a starting point for this method. 


\section{Examples of del Pezzo orders on the projective plane}

\subsection{Del Pezzo order ramified on a smooth quartic}

The first explicit example of a moduli space of $\mathcal{A}$-line bundles was given in [CK11]. In that example $\mathcal{A}$ should be a maximal order of rank four on $\mathbb{P}^{2}$ ramified on a smooth quartic $D$. The idea is to use the noncommutative cyclic covering trick defined in (1.3).

This constructs $\mathcal{A}$ as a cyclic algebra $A=A\left(Y, L_{\sigma}, \phi\right)$ on a double cover $Y$ of $\mathbb{P}^{2}$. So any $\mathcal{A}$-line bundle on $\mathbb{P}^{2}$ comes from a locally free sheaf of rank two on $Y$. The problem of studying locally free sheaves of rank four on $\mathbb{P}^{2}$ reduces to the study of locally free sheaves of rank two on $Y$ with certain properties.

\section{Remark 4.1:}

The map $\pi: X \rightarrow Y$ used in the noncommutative cyclic covering trick is finite. Thus $\pi$ is especially an affine morphism so that $\pi_{*}$ induces an equivalence from the category of quasi-coherent $\mathcal{O}_{X}$-modules on $X$ and the category of quasi-coherent $\pi_{*} \mathcal{O}_{X}$-modules on $Y$, and since $\pi$ is finite this equivalence maps coherent locally free $\mathcal{O}_{X}$-modules to coherent locally free $\pi_{*} \mathcal{O}_{X}$-modules. Furthermore we have for all $i \geq 0$ and all quasi-coherent sheaves $F$ on $X$ an isomorphism $H^{i}(X, F) \cong H^{i}\left(Y, \pi_{*} F\right)$, see [Har77, Excercise III.4.1]. So all cohomology groups of $\mathcal{A}$-modules in question can also be computed on $X$. Because of this we omit the notation of $\pi_{*}$ and will work completely on $X$.

We will now summarize the results of [CK11] to see if we can obtain similar results in other cases:

Assume $D$ is a smooth quartic curve in $\mathbb{P}^{2}$. There is a double cover $\pi: Y \rightarrow \mathbb{P}^{2}$ ramified on $D$, see [WCdV84, Lemma 17.1]. The Galois group $G$ of $\pi$ is generated by an element $\sigma$ of order 2, the so-called Geisser involution. Using $K_{Y}=\pi^{*} K_{\mathbb{P}^{2}}+R$, where $\mathrm{R}$ is the ramification divisor in $Y$, one can show that $K_{Y}^{2}=2$ so that $Y$ can also be seen as the blow up of $\mathbb{P}^{2}$ at 7 points $\left\{P_{i}\right\}_{1 \leq i \leq 7}$ in general position. We have the morphism $\phi: Y \rightarrow \mathbb{P}^{2}$ which contracts the exceptional divisors. It is known that $Y$ contains $56(-1)$-curves, they are given by:

- the exceptional divisors $E_{i}$ correpsonding to $P_{i}$ for $i=1, \ldots, 7$;

- the strict transforms $L_{i j}$ of the lines containing two points $P_{i}$ and $P_{j}$ for $1 \leq i<j \leq 7$;

- the strict transforms $C_{i j}$ of the conics containg all points except $P_{i}$ and $P_{j}$ for $1 \leq i<j \leq 7$;

- the strict transforms $D_{i}$ of the cubics passing to all points with a double point at $P_{i}$.

We have the following facts about $Y$ :

- $\operatorname{Pic}(Y)=\mathbb{Z}^{8}$ generated by the $E_{i}$ for $i=1, \ldots, 7$ and $H=\phi^{*} l$ where $l \subset \mathbb{P}^{2}$ is a line;

- $K_{Y}=-\pi^{*} l=-3 H+\sum_{i=1}^{7} E_{i}$

The 56 curves can also be described in the following way:

It is known that the quartic $D$ has 28 bitangents $l_{i}$, so that the preimage $H_{i}=\pi^{-1}\left(l_{i}\right)$ decomposes into two (-1)-curves. Since $\pi \circ \sigma=\pi$ we see that each $H_{i}$ is $G$-invariant, so that the decomposition must look like $H_{i}=C_{i} \cup \sigma\left(C_{i}\right)$. The 56 curves come in 28 pairs $\left(C_{i}, \sigma(C i)\right)$. To find the action of $\sigma$ on $\operatorname{Pic}(X)$ we need to determine the images of the exceptional divisors $\left\{E_{i}\right\}_{1 \leq i \leq 7}$ and $H$ under $\sigma$. To do this the following lemma helps: 


\section{Lemma 4.2 ([DO88, Section VII.4]):}

Assume $\pi: Y \rightarrow \mathbb{P}^{2}$ is a double cover ramified on a smooth quartic. If we denote the (-1)-curves in $Y$ as described above and let $G=<\sigma>$ be the Galois group of $\pi$ then we have:

$$
\sigma\left(E_{i}\right)=D_{i}, \sigma\left(L_{i j}\right)=C_{i j} \text { and } \sigma(H)=8 H-3 \sum_{i=1}^{7} E_{i} .
$$

Since $D_{i}=3 H-2 E_{i}-\sum_{j \neq i} E_{j}$ in $\operatorname{Pic}(X)$ we can finally see that the action of $G$ on $\operatorname{Pic}(Y)$ with respect to the basis $\left\{H, E_{1}, \ldots, E_{7}\right\}$ is given by:

$$
\operatorname{Pic}(\sigma)=\left(\begin{array}{rrrrrrrr}
8 & 3 & 3 & 3 & 3 & 3 & 3 & 3 \\
-3 & -2 & -1 & -1 & -1 & -1 & -1 & -1 \\
-3 & -1 & -2 & -1 & -1 & -1 & -1 & -1 \\
-3 & -1 & -1 & -2 & -1 & -1 & -1 & -1 \\
-3 & -1 & -1 & -1 & -2 & -1 & -1 & -1 \\
-3 & -1 & -1 & -1 & -1 & -2 & -1 & -1 \\
-3 & -1 & -1 & -1 & -1 & -1 & -2 & -1 \\
-3 & -1 & -1 & -1 & -1 & -1 & -1 & -2
\end{array}\right)
$$

Using methods from linear algebra we can deduce the following lemma:

Lemma 4.3 ([Cha05, Chapter 6]):

Assume $\pi: Y \rightarrow \mathbb{P}^{2}$ is the double cover ramified on a smooth quartic D. If $G=\langle\sigma\rangle$ is the Galois group of $\pi$, then:

1. $H^{0}(G, \operatorname{Pic}(Y))=\operatorname{Pic}(Y)^{G}=<\pi^{*} l>=\pi^{*} \operatorname{Pic}\left(\mathbb{P}^{2}\right) ;$

2. $H^{1}(G, \operatorname{Pic}(Y))=(\mathbb{Z} / 2 \mathbb{Z})^{6}$ and this group is generated by $E_{i}-E_{j}$ for $i \neq j$;

3. if $E$ and $E^{\prime}$ are exceptional curves in $Y$, then $E-E^{\prime} \in H^{1}(G, P i c(Y))$.

We pick two disjoint exceptional curves $E$ and $E^{\prime}$, then $L:=\mathcal{O}_{Y}\left(E-E^{\prime}\right)$ has the property that $\phi: L_{\sigma}^{2} \stackrel{\sim}{\longrightarrow} \mathcal{O}_{Y}$ is an isomorphism and that this relation satisfies the overlap condition. So $A=\mathcal{O}_{Y} \oplus L_{\sigma}$ defines a terminal maximal order $\mathcal{A}$ on $\mathbb{P}^{2}$ ramified on $D$.

Since $\mathcal{A}$-modules are locally free sheaves of rank two on $Y$, one can look at their Chern classes on $Y$. The next proposition will determine the possible first Chern classes of $A$-line bundles. In the following $H$ will denote the pullback of a general line $l \subset \mathbb{P}^{2}$ under $\pi$.

Proposition 4.4 ([CK11, Proposition 5.1]):

Assume $M$ is an $A$-line bundle, then there is an $n \in \mathbb{Z}$ such that $c_{1}(M)=L \otimes \mathcal{O}_{Y}(n H)$.

One can show that it is enough to consider the cases $n=0$ and $n=1$, see [Ler11, Remark 3.8]. In these cases one can find the minimal $c_{2}$ :

Proposition 4.5 ([CK11, Proposition 5.2]):

Assume $M$ is an $A$-line bundle with first Chern class $c_{1}=L \otimes \mathcal{O}_{Y}(n H)$ with $n=0$ or $n=1$, then the minimal $c_{2}$ is given by 0 respectively 1 . 
Theorem 4.6 ([CK11, Proposition 6.10]):

Assume $M$ is an A-line bundle with $c_{1}=L$ and $c_{2}=0$, then $M \cong A$. The coarse moduli space $\operatorname{Pic}(A)$ of such line bundles is a point.

Lemma 4.7 ([CK11, Theorem 6.10]):

Assume $M$ is an A-line bundle with $c_{1}=L \otimes \mathcal{O}_{Y}(H)$ and $c_{2}=1$. Then $M$ sits in an exact sequence

$$
0 \longrightarrow \mathcal{O}_{Y} \longrightarrow M \longrightarrow I_{p} \mathcal{O}_{Y}\left(E+\sigma E^{\prime}\right) \longrightarrow 0
$$

for some $p \in Y$. (Here $I_{p}$ is the ideal sheaf of $p$ and $E+\sigma E^{\prime}=E-E^{\prime}+E^{\prime}+\sigma E^{\prime}=E-E^{\prime}+H$ ).

The sheaf $M$ in the middle of this sequence is locally free due to the Serre correspondence for codimension two subsets, see for example [HL10, Theorem 5.1.1]. Using this knowledge, a family of $\mathcal{O}_{Y}$-modules over a rational curve $C \subset Y$ is constructed in [CK11, Lemma 6.8], which exhibits the moduli space as a double cover of $C$ ramified at 6 points. This leads to the following result:

Theorem 4.8 ([CK11, Theorem 6.11]):

The coarse moduli scheme Pic $(A)$ of A-line bundles with Chern classes $c_{1}=L \otimes \mathcal{O}_{Y}(H)$ and $c_{2}=1$ is a smooth projective curve of genus 2.

\subsection{Del Pezzo order ramified on two conics}

The second explicit computation was done in [Ler11] for an order $\mathcal{A}$ ramified on a union of two conics, intersecting in four distinct points. So the ramification locus is singular in this case. We will summarize the result from the mentioned article:

Assume $E \subset \mathbb{P}^{2}$ is a smooth conic and look at the double cover $\pi: Y \rightarrow \mathbb{P}^{2}$ ramified on $E$. Let $G=<\sigma>$ be the Galois group of $\pi$.

Then the following facts are well known:

- $Y \cong \mathbb{P}^{1} \times \mathbb{P}^{1}$

- $\operatorname{Pic}(Y)=\mathbb{Z} \oplus \mathbb{Z}$;

- $\sigma$ acts on $\operatorname{Pic}(Y)$ via $\sigma^{*}\left(\mathcal{O}_{Y}(n, m)\right)=\mathcal{O}_{Y}(m, n)$.

Let $H=\pi^{*} l$ for a general line $l \subset \mathbb{P}^{2}$, then $H$ is an ample $(1,1)$-divisor.

We choose another smooth conic $E^{\prime}$ intersecting $E$ in four distinct points and set $D:=\pi^{*} E^{\prime}$, then $D$ is a smooth $(2,2)$-divisor.

Let $L=\mathcal{O}_{Y}(-1,-1)$ and fix a morphism $\phi: L_{\sigma}^{2} \stackrel{\sim}{\rightarrow} \mathcal{O}_{Y}(-D) \hookrightarrow \mathcal{O}_{Y}$. One can show that $\phi$ satisfies the overlap condition. Thus $A=A\left(Y, L_{\sigma}, \phi\right)$ defines an order $\mathcal{A}$ on $\mathbb{P}^{2}$ ramified on $E \cup E^{\prime}$ and one can show that $\mathcal{A}$ is maximal and terminal.

To begin, we check the possible first Chern classes of $A$-line bundles on $Y$.

Proposition 4.9 ([Ler11, Proposition 3.4]):

Assume $M$ is an $A$-line bundle, then there is some $n \in \mathbb{Z}$ such that $c_{1}(M)=\mathcal{O}_{Y}(n, n)$.

Again one can see that it is enough to consider just two cases, here they are $n=-1$ and $n=-2$. 
Proposition 4.10 ([Ler11, Proposition 3.6]):

Assume $M$ is an $A$-line bundle with first Chern class $c_{1}=\mathcal{O}_{Y}(n, n)$ where $n=-1$ or $n=-2$, then the minimal $c_{2}$ is given by 0 respectively 2 .

Theorem 4.11 ([Ler11, Theorem 3.11]):

Assume $M$ is an A-line bundle with $c_{1}=\mathcal{O}_{Y}(-1,-1)$ and $c_{2}=0$, then $M \cong A$. The coarse moduli space Pic $(A)$ of such line bundles is a point.

For the second pair of Chern classes one first checks how the $\mathcal{O}_{Y}$-module structure of an $A$-line bundle looks like.

Theorem 4.12 ([Ler11, Theorem 3.12]):

Assume $M$ is an A-line bundle with $c_{1}=\mathcal{O}_{Y}(-2,-2)$ and $c_{2}=2$. Then we have either

$$
M \cong \mathcal{O}_{Y}(-1,-1) \oplus \mathcal{O}_{Y}(-1,-1)
$$

as an $\mathcal{O}_{Y}$-module, or

$$
M \cong A \otimes \mathcal{O}_{Y} \mathcal{O}_{Y}(-F)
$$

as A-modules, where $F$ is either an $(1,0)$-or a $(0,1)$-divisor.

Next the so-called Hilbert scheme $\operatorname{Hilb}(A)$ of $A$ is constructed in [Ler11]. This is the moduli space of left sided quotients of $A$ with some fixed numerical data. With a lot of work it is proven that this moduli scheme is connected and hence:

Theorem 4.13 ([Ler11, Section 4.2]):

The Hilbert scheme Hilb(A) of left sided quotients with $c_{1}=\mathcal{O}_{Y}(1,1)$ and $c_{2}=2$ is a smooth projective surface.

One observes that the kernel of such a quotient is actually an $A$-line bundle with $c_{1}=\mathcal{O}_{Y}(-2,-2)$ and $c_{2}=2$, so that there is a map from the Hilbert scheme to the coarse moduli space of such $A$-line bundles, giving this interesting theorem:

Theorem 4.14 ([Ler11, Theorem 5.1]):

The Hilbert scheme Hilb(A) of left sided quotients with $c_{1}=\mathcal{O}_{Y}(1,1)$ and $c_{2}=2$ is a ruled surface over the coarse moduli space Pic $(A)$ of $A$-line bundles with $c_{1}=\mathcal{O}_{Y}(-2,-2)$ and $c_{2}=2$.

Then it is finally shown, using the previous theorem, that:

Theorem 4.15 ([Ler11, Theorem 5.2]):

The coarse moduli space Pic $(A)$ of A-line bundles with $c_{1}=\mathcal{O}_{Y}(-2,-2)$ and $c_{2}=2$ is a smooth projective curve of genus 2. 


\subsection{Del Pezzo orders ramified on a smooth cubic curve}

In the last two sections we have seen moduli spaces of $\mathcal{A}$-line bundles, where $\mathcal{A}$ is a terminal del Pezzo order on $\mathbb{P}^{2}$ with a ramification divisor of degree four. Looking at the classification of terminal del Pezzo orders on $\mathbb{P}^{2}$ in (1.25), we see that there should also be del Pezzo orders with smooth ramification divisor of degree three and five.

Unfortunately it is impossible to construct such an order with ramification divisor of degree five using the noncommutative cyclic covering trick, since the ramification index $e$ has to be two in this case and there is no double cover ramified on a quintic.

But we can construct an order ramified on a smooth cubic using the noncommutative cyclic covering trick. We have to use a triple cover $Y$ of $\mathbb{P}^{2}$ leading to locally free sheaves of rank three on $Y$ and therefore $\mathcal{A}$-line bundles of rank nine on $\mathbb{P}^{2}$.

Assume $D \subset \mathbb{P}^{2}$ is a smooth cubic curve. We pick a triple cover $\pi: Y \rightarrow \mathbb{P}^{2}$ ramified on $D$. The Galois group $G$ of $\pi$ is given by $G=<\sigma>=\left\{i d_{Y}, \sigma, \sigma^{2}\right\}$.

First we compute the canonical divisor of $Y$ :

There is the well known formula:

$$
K_{Y}=\pi^{*} K_{\mathbb{P}^{2}}+R,
$$

where $R \subset Y$ is the ramification divisor of $\pi$. Using [WCdV84, Lemma I.17.1] we see that $R=2 \pi^{*} l$ for a general line $l \subset \mathbb{P}^{2}$. As $K_{\mathbb{P}^{2}}=-3 l$, we conclude that $K_{Y}=-\pi^{*} l$.

Since $\pi$ is a triple cover, $\pi_{*} \pi^{*}$ is multiplication by three on divisors, consequently we get:

$$
K_{Y}^{2}=\pi^{*} l . \pi^{*} l=\left(\pi_{*} \pi^{*} l\right) . l=3 .
$$

This shows that $K_{Y}^{2}=K_{\mathbb{P}^{2}}^{2}-6$, and so $Y$ can also be seen as the blow up of $\mathbb{P}^{2}$ at 6 points $\left\{P_{i}\right\}_{1 \leq i \leq 6}$.

Such a blow up $\psi: Y \rightarrow \mathbb{P}^{2}$ is known to be a smooth cubic surface. A smooth cubic surface contains 27 lines which are in fact the 27 irreducible (-1)-curves of the blow up.

Theorem 4.16 ([Har77, Theorem V.4.9]):

The smooth cubic surface $Y$ contains exactly 27 lines, which are the 27 irreducible (-1)-curves. They are given by:

- the exceptional divisors $E_{i}$ for $i=1, \ldots, 6$;

- the strict transforms $F_{i j}$ of the lines in $\mathbb{P}^{2}$ containing $P_{i}$ and $P_{j}$ for $1 \leq i<j \leq 6$;

- the strict transforms $G_{j}$ of the conics in $\mathbb{P}^{2}$ containing five $P_{i}$ for $i \neq j, j=1, \ldots, 6$.

Let $L$ be the pullback of a general line $l \subset \mathbb{P}^{2}$ under $\psi$, then we know the following facts about $Y$, see [Har77, Proposition V.4.8]:

- $\operatorname{Pic}(Y)=\mathbb{Z}^{7}$, generated by the classes of $E_{1}, \ldots, E_{6}$ for $i=1, \ldots, 6$ and $L$;

- the intersection pairing is given by: $L^{2}=1, E_{i}^{2}=-1, L E_{i}=0$ and $E_{i} E_{j}=0$ for $i \neq j$;

- the canonical divisor is $K_{Y}=-\pi^{*} l=-3 L+\sum_{i=1}^{6} E_{i}$. 
It is also known that $D$ has 9 inflection points, so there are 9 inflection lines $l_{i}$ tangent to $D$. Thus $\pi^{-1}\left(l_{i}\right)$ decomposes as a union of three irreducible $(-1)$-curves $\pi^{-1}\left(l_{i}\right)=C_{1}^{i} \cup C_{2}^{i} \cup C_{3}^{i}$ meeting in one point, a so-called Eckardt point of $Y$. As $\pi$ is $G$-invariant the decomposition must be given by $\pi^{-1}\left(l_{i}\right)=C_{i} \cup \sigma\left(C_{i}\right) \cup \sigma^{2}\left(C_{i}\right)$. So the 27 lines on $Y$ come in nine 3 -tuples $\left(C_{i}, \sigma\left(C_{i}\right), \sigma^{2}\left(C_{i}\right)\right)$.

To find an explicit blow up model for $Y$ starting from the triple cover description and to understand the behaviour of the lines on $Y$ with respect to the Galois group $G$ we will use the following proposition:

Proposition 4.17 ([Har77, Proposition 4.10]):

Let $Y$ be a smooth cubic surface and let $E_{1}, \ldots, E_{6}$ be six mutually skew lines on $Y$. Then there is a morphism $\phi: Y \rightarrow \mathbb{P}^{2}$ making $Y$ isomorphic to the blow up of $\mathbb{P}^{2}$ in six points $P_{1}, \ldots, P_{6}$ (no 3 collinear and not all 6 on a conic), such that $E_{1}, \ldots, E_{6}$ are the exceptional divisors for $\phi$.

So assume $\pi: Y \rightarrow \mathbb{P}^{2}$ is the triple cover ramified on $D$, then we choose 6 mutually skew lines $E_{1}, \ldots, E_{6}$ on $Y$ necessarily lying over distinct inflection lines and use the previous proposition to obtain a blow up model $\phi: Y \rightarrow \mathbb{P}^{2}$.

\section{Remark 4.18:}

We can easily deduce the incidence relations among the 27 lines on $Y$, see [Har77, Remark V.4.10.1]:

- $E_{i} \cap E_{j}=\emptyset$ if $i \neq j$;

- $E_{i} \cap F_{j k} \neq \emptyset$ if and only if $i=j$ or $i=k$;

- $E_{i} \cap G_{j} \neq \emptyset$ if and only if $i \neq j$;

- $F_{i j} \cap F_{k l} \neq \emptyset$ if and only if $i, j, k, l$ are all distinct;

- $F_{i j} \cap G_{k} \neq \emptyset$ if and only if $i=k$ or $j=k$;

- $G_{i} \cap G_{j}=\emptyset$ for $i \neq j$.

Now we want to understand the action of $G$ on $\operatorname{Pic}(Y)$. To do that, we need to understand the six 3 -tuples given by $\left(E_{i}, \sigma\left(E_{i}\right), \sigma^{2}\left(E_{i}\right)\right)$ for $i=1, \ldots, 6$.

\section{Lemma 4.19:}

Assume $E_{i}$ is an exceptional divisor of $\phi: Y \rightarrow \mathbb{P}^{2}$, then there exists a permutation $\tau \in S_{6}$ without fixed points such that:

$$
\left\{\sigma\left(E_{i}\right), \sigma^{2}\left(E_{i}\right)\right\}=\left\{F_{i \tau(i)}, G_{\tau(i)}\right\} \text { or }\left\{\sigma\left(E_{i}\right), \sigma^{2}\left(E_{i}\right)\right\}=\left\{F_{\tau(i) i}, G_{\tau(i)}\right\} .
$$

\section{Proof:}

Since $\pi^{*} l=3 L-\sum_{i=1}^{6} E_{i}$ we see that $E_{i} \cdot \pi^{*} l=1$, so we have

$$
E_{i} \cdot \pi^{*}\left(l_{i}\right)=E_{i} \cdot\left(E_{i}+\sigma\left(E_{i}\right)+\sigma^{2}\left(E_{i}\right)\right)=1 .
$$

Using $E_{i}^{2}=-1$ and $E_{i} \cdot \sigma^{j}\left(E_{i}\right) \in\{0,1\}$ for $j=1,2$ we see that: 


$$
E_{i} \cdot \sigma\left(E_{i}\right)=E_{i} \cdot \sigma^{2}\left(E_{i}\right)=1 .
$$

The properties of the intersection pairing then show that $\sigma\left(E_{i}\right) \cdot \sigma^{2}\left(E_{i}\right)=1$.

Accordingly we have:

$$
E_{i} \cap \sigma\left(E_{i}\right) \neq \emptyset \text { and } E_{i} \cap \sigma^{2}\left(E_{i}\right) \neq \emptyset .
$$

The incidence relations and the fact that $E_{i}, \sigma\left(E_{i}\right)$ and $\sigma^{2}\left(E_{i}\right)$ meet in one point now imply the following:

$$
\left\{\sigma\left(E_{i}\right), \sigma^{2}\left(E_{i}\right)\right\}=\left\{F_{i j}, G_{k}\right\} \text { or }\left\{\sigma\left(E_{i}\right), \sigma^{2}\left(E_{i}\right)\right\}=\left\{F_{j i}, G_{k}\right\}
$$

for some $j$ and $k$ with $j, k \neq i$.

But we also know $\sigma\left(E_{i}\right) \cap \sigma^{2}\left(E_{i}\right) \neq \emptyset$ so that $j$ and $k$ must conincide.

Applying this method to all exceptional divisors $E_{i}$ for $i=1, \ldots, 6$ we see that there exists an element $\tau \in S_{6}$ without fixed points such that

$$
\left\{\sigma\left(E_{i}\right), \sigma^{2}\left(E_{i}\right)\right\}=\left\{F_{i \tau(i)}, G_{\tau(i)}\right\}
$$

if $i<\tau(i)$ or

$$
\left\{\sigma\left(E_{i}\right), \sigma^{2}\left(E_{i}\right)\right\}=\left\{F_{\tau(i) i}, G_{\tau(i)}\right\}
$$

if $\tau(i)<i$.

Since we now fully understand the set $\left\{E_{i}, \sigma\left(E_{i}\right), \sigma^{2}\left(E_{i}\right)\right\}$, we can find the action of $G$ on $\operatorname{Pic}(Y)$ :

\section{Lemma 4.20:}

Assume $\pi: Y \rightarrow \mathbb{P}^{2}$ is a triple cover ramified on a smooth cubic D. If $G$ is the Galois group of $\pi$, then $G$ acts on Pic $(Y)$ via:

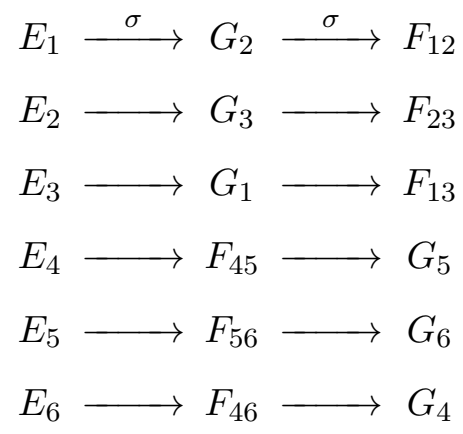

\section{Proof:}

The lines $E_{1}, \ldots, E_{6}$ are mutually skew, so the six lines $\sigma\left(E_{1}\right), \ldots, \sigma\left(E_{6}\right)$ must also be mutually skew and the six lines $\sigma^{2}\left(E_{1}\right), \ldots, \sigma^{2}\left(E_{6}\right)$ as well.

By (4.19) we know that:

$$
\left\{\sigma\left(E_{i}\right), \sigma^{2}\left(E_{i}\right)\right\}=\left\{F_{i \tau(i)}, G_{\tau(i)}\right\} \text { or }\left\{\sigma\left(E_{i}\right), \sigma^{2}\left(E_{i}\right)\right\}=\left\{F_{\tau(i) i}, G_{\tau(i)}\right\}
$$

for a permutation $\tau \in S_{6}$.

With the help of the incidence relations we see that $\sigma\left(E_{1}\right), \ldots, \sigma\left(E_{6}\right)$ and $\sigma^{2}\left(E_{1}\right), \ldots, \sigma^{2}\left(E_{6}\right)$ each contain three of the $F_{i \tau(i)}$ and three of the $G_{i}$. So up to a permutation of the six lines we can choose $\tau=(123)(456)$ and see that the map is given as described. 
To compute the matrix of $\operatorname{Pic}(\sigma)$ with respect to the basis $\left\{L, E_{1}, \ldots, E_{6}\right\}$ we need to determine the image of $L$ under $\sigma$.

Since $F_{45}$ is the strict transform of the line containing $P_{4}$ and $P_{5}$, we know that we have

$$
F_{45}=L-E_{4}-E_{5} \text { in } \operatorname{Pic}(Y) .
$$

Consequently we get:

$$
\sigma\left(F_{45}\right)=\sigma\left(L-E_{4}-E_{5}\right)=\sigma(L)-\sigma\left(E_{4}\right)-\sigma\left(E_{5}\right) .
$$

Using (4.20) we know $\sigma\left(F_{45}\right), \sigma\left(E_{4}\right)$ and $\sigma\left(E_{5}\right)$ in $\operatorname{Pic}(Y)$. Putting everything together we get:

$$
\sigma(L)=4 L-\left(E_{1}+E_{2}+E_{3}\right)-2\left(E_{4}+E_{5}+E_{6}\right) .
$$

As we have $F_{i j}=L-E_{i}-E_{j}$ and $G_{i}=2 L-\sum_{j \neq i} E_{j}$ in $\operatorname{Pic}(Y)$ we see that the action of $G$ on $\operatorname{Pic}(Y)$ with respect to the basis $\left\{L, E_{1}, \ldots, E_{6}\right\}$ is given by:

$$
\operatorname{Pic}(\sigma)=\left(\begin{array}{rrrrrrr}
4 & 2 & 2 & 2 & 1 & 1 & 1 \\
-1 & -1 & -1 & 0 & 0 & 0 & 0 \\
-1 & 0 & -1 & -1 & 0 & 0 & 0 \\
-1 & -1 & 0 & -1 & 0 & 0 & 0 \\
-2 & -1 & -1 & -1 & -1 & 0 & -1 \\
-2 & -1 & -1 & -1 & -1 & -1 & 0 \\
-2 & -1 & -1 & -1 & 0 & -1 & -1
\end{array}\right)
$$

If we denote the matrix by $Z$, then one can easily verify that:

- $Z^{2}=\operatorname{Pic}\left(\sigma^{2}\right)$;

- $Z^{3}=\operatorname{Pic}\left(\sigma^{3}\right)=\operatorname{Pic}\left(i d_{Y}\right)=i d_{P i c(Y)}$.

To find a maximal order $\mathcal{A}$ on $\mathbb{P}^{2}$ ramified on $D$, we need to find $L \in \operatorname{Pic}(Y)$ with a relation $\phi$ : $L_{\sigma}^{3} \stackrel{\sim}{\longrightarrow} \mathcal{O}_{Y}$ which satisfies the overlap condition. For this purpose we compute $H^{1}(G, \operatorname{Pic}(Y))$. To do this we need the following two matrices:

$$
\begin{array}{r}
1+Z+Z^{2}=\left(\begin{array}{rrrrrrr}
9 & 3 & 3 & 3 & 3 & 3 & 3 \\
-3 & -1 & -1 & -1 & -1 & -1 & -1 \\
-3 & -1 & -1 & -1 & -1 & -1 & -1 \\
-3 & -1 & -1 & -1 & -1 & -1 & -1 \\
-3 & -1 & -1 & -1 & -1 & -1 & -1 \\
-3 & -1 & -1 & -1 & -1 & -1 & -1 \\
-3 & -1 & -1 & -1 & -1 & -1 & -1
\end{array}\right) \\
1-Z=\left(\begin{array}{rrrrrrr}
-3 & -2 & -2 & -2 & -1 & -1 & -1 \\
1 & 2 & 1 & 0 & 0 & 0 & 0 \\
1 & 0 & 2 & 1 & 0 & 0 & 0 \\
1 & 1 & 0 & 2 & 0 & 0 & 0 \\
2 & 1 & 1 & 1 & 2 & 0 & 1 \\
2 & 1 & 1 & 1 & 1 & 2 & 0 \\
2 & 1 & 1 & 1 & 0 & 1 & 2
\end{array}\right)
\end{array}
$$


Using linear algebra we can deduce the following result:

\section{Lemma 4.21:}

Assume $\pi: Y \rightarrow \mathbb{P}^{2}$ is a triple cover ramified on a smooth cubic curve. If $G=\langle\sigma\rangle$ is the Galois group of $\pi$ then:

1. $H^{0}(G, \operatorname{Pic}(Y))=\operatorname{ker}(1-Z)=\operatorname{Pic}(Y)^{G}=<3 L-\sum_{i=1}^{6} E_{i}>=<\pi^{*} l>=\pi^{*} \operatorname{Pic}\left(\mathbb{P}^{2}\right) ;$

2. $\operatorname{ker}\left(1+Z+Z^{2}\right)=<L-3 E_{1}, E_{i}-E_{i+1}$ for $i=1, \ldots, 5>=\mathbb{Z}^{6}$.

So especially the difference of two exceptional divisors is in $H^{1}(G, \operatorname{Pic}(Y))$.

We will only mention that one can compute $i m(1-Z)=\mathbb{Z}^{6}$ and using the Smith normal form over the principal ideal domain $\mathbb{Z}$, one gets:

$$
H^{1}(G, \operatorname{Pic}(Y))=\operatorname{ker}\left(1+A+A^{2}\right) / \operatorname{im}(1-A)=\mathbb{Z} / 3 \mathbb{Z} \times \mathbb{Z} / 3 \mathbb{Z} .
$$

We recall that the Hirzebruch-Riemann-Roch formula for rank three bundles $M$ on $Y$ is given by:

$$
\chi(M)=\frac{c_{1}^{2}-c_{1} K_{Y}}{2}-c_{2}+3
$$

\subsubsection{Construction of an order}

Using the results of the previous section, we choose the line bundle $L=\mathcal{O}_{Y}\left(E_{1}-E_{2}\right)$. Then we have an isomorphism $\phi: L_{\sigma}^{3} \stackrel{\sim}{\longrightarrow} \mathcal{O}_{Y}$ which satisfies the overlap condition. Thus the algebra $A=\mathcal{O}_{Y} \oplus L_{\sigma} \oplus L_{\sigma}^{2}$ is a cyclic algebra and defines a del Pezzo order of rank nine on $\mathbb{P}^{2}$ ramified on the cubic curve $D$.

Because we have $L_{\sigma}^{2}=\left(L \otimes \mathcal{O}_{Y} \sigma^{*} L\right)_{\sigma^{2}}$, we see that the Chern classes of $A$ are given by:

$$
\begin{aligned}
& c_{1}(A)=2\left(E_{1}-E_{2}\right)+\sigma^{*}\left(E_{1}-E_{2}\right) \\
& c_{2}(A)=-1
\end{aligned}
$$

If we define $H:=\pi^{*} l$ for a general line $l \subset \mathbb{P}^{2}$, then the ramification divisor $R$ of $\pi$ on $Y$ is given by $2 H$. Using this and the result [CK11, Proposition 4.1], we see that the $\mathcal{O}_{Y}$-module associated to $\omega_{A}$ is given by:

$$
\omega_{\mathbb{P}^{2}} \otimes_{\mathcal{O}_{\mathbb{P}^{2}}}\left(A \otimes_{\mathcal{O}_{Y}} \mathcal{O}_{Y}(R)\right) \cong \mathcal{O}_{Y}(-3 H) \otimes_{\mathcal{O}_{Y}}\left(A \otimes_{\mathcal{O}_{Y}} \mathcal{O}_{Y}(2 H)\right) \cong A \otimes_{\mathcal{O}_{Y}} \mathcal{O}_{Y}(-H),
$$

where the first isomorphism is the projection formula for $\pi_{*}$.

We note that $c_{1}\left(\omega_{A}\right)=c_{1}(A)-3 H$. This result will be needed later.

\subsubsection{Possible Chern classes}

Since $L_{\sigma}^{3} \cong \mathcal{O}_{Y}$ we can use the argument given in [CK11, Theorem 3.3] to see the following: if $M$ is an $A$-line bundle then we must have $L_{\sigma} \otimes M \cong M$, thus implying:

$$
L \otimes \mathcal{O}_{Y} \sigma^{*} M \cong M .
$$

This observation leads to the following lemma. 


\section{Lemma 4.22:}

If $M$ is an A-line bundle, then $c_{1}(M)=c_{1}(A)+n H$ for some $n \in \mathbb{Z}$.

\section{Proof:}

Using the isomorphism (17) and applying $c_{1}$ shows that

$$
3 c_{1}(L)+c_{1}\left(\sigma^{*} M\right)=c_{1}(M)
$$

respectively

$$
c_{1}(M)-\sigma^{*} c_{1}(M)=3\left(E_{1}-E_{2}\right) .
$$

We see that if we have two solutions to the last equation for $c_{1}(M)$, then their difference is an element of $\operatorname{ker}\left(1-\sigma^{*}\right)=\operatorname{Pic}(Y)^{G}$. But we saw that $\operatorname{Pic}(Y)^{G}=\pi^{*} \operatorname{Pic}\left(\mathbb{P}^{2}\right)=\mathbb{Z} H$.

One solution to this equation is given by $c_{1}(M)=c_{1}(A)=2\left(E_{1}-E_{2}\right)+\sigma^{*}\left(E_{1}-E_{2}\right)$, since $L_{\sigma}^{3} \cong \mathcal{O}_{Y}$ implies that $E_{1}-E_{2}$ is equivalent to $-\sigma^{*}\left(E_{1}-E_{2}\right)-\sigma^{2^{*}}\left(E_{1}-E_{2}\right)$ in $\operatorname{Pic}(Y)$.

So if $M$ is an $A$-line bundle then there is an $n \in \mathbb{Z}$ such that $c_{1}(M)=c_{1}(A)+n H$.

The next lemma gives a vanishing criterion for the second cohomology group of an $A$-line bundle. This will be helpful when we are working with the Euler characteristic.

\section{Lemma 4.23:}

Let $M$ be an A-line bundle with $c_{1}(M)=c_{1}(A)+n H$ and $n \geq-2$. Then we have $H^{2}(Y, M)=0$.

\section{Proof:}

Since $M$ is an $A$-module, we have

$$
H^{2}(Y, M)=H^{2}\left(Y, \mathcal{H o m}_{A}(A, M)\right)=\operatorname{Ext}_{A}^{2}(A, M) .
$$

Using Serre duality shows that we have

$$
H^{2}(Y, M)=\operatorname{Hom}_{A}\left(M, \omega_{A}\right)^{\prime} .
$$

Now if $\operatorname{Hom}_{A}\left(M, \omega_{A}\right)$ is non-empty, then by $(1.80) c_{1}\left(\omega_{A}\right)-c_{1}(M)$ must be effective, but this divisor is

$$
c_{1}(A)-3 H-c_{1}(A)-n H=-(n+3) H .
$$

By the choice of $n$ this divisor cannot be effective, so $\operatorname{Hom}_{A}\left(M, \omega_{A}\right)=0$, which implies that $H^{2}(Y, M)=0$.

Again it is enough to consider the cases where $n \in\{0,1,2\}$. For these cases we will now try to find the minimal $c_{2}$.

\section{Lemma 4.24:}

If $M$ is an A-line bundle with $c_{1}=c_{1}(A)$, then the minimal second Chern class is $c_{2}=-1$.

\section{Proof:}

One first computes $c_{1}(A)^{2}=-6$ and $c_{1}(A) . H=0$. So if $M$ is an $A$-line bundle with $c_{1}=c_{1}(A)$ and some $c_{2}$ then Hirzebruch-Riemann-Roch shows that: 


$$
\chi(M)=\frac{-6}{2}-c_{2}+3=-c_{2} .
$$

Now we assume there is an $A$-line bundle $M$ with $c_{2}(M)<-1$.

Then we must have $\chi(M)>1$. But (4.23) shows that $H^{2}(Y, M)=0$ so we must have $\operatorname{dim}\left(H^{0}(Y, M)\right)>1$.

This implies that there is an embedding $A \hookrightarrow M$, and since $c_{1}(M)=c_{1}(A)$ this must be an isomorphism since both bundles are locally free.

But this is impossible since $c_{2}(M) \neq c_{2}(A)$. We conclude that such an $A$-line bundle cannot exist, implying $c_{2}=-1$ is minimal.

\section{Lemma 4.25:}

If $M$ is an A-line bundle with $c_{1}=c_{1}(A)+H$, then the minimal second Chern class is $c_{2}=0$.

\section{Proof:}

Assume there is an $A$-line bundle $M$ with $c_{1}=c_{1}(A)+H$ and $c_{2}(M)=m<0$.

Define a rank three vector bundle $N$ by $N:=M \otimes_{\mathcal{O}_{Y}} \mathcal{O}_{Y}\left(-\sigma^{2}\left(E_{2}\right)\right)$.

We compute the Chern classes of $N$ by using (1.79) and get:

$$
c_{1}(N)=c_{1}(M)-3 \sigma^{2}\left(E_{2}\right) \text { and } c_{2}(N)=c_{2}(M)-2 c_{1}(M) \cdot \sigma^{2}\left(E_{2}\right)+3\left(\sigma^{2}\left(E_{2}\right)\right)^{2}
$$

Since we know all possible intersection products we see that:

$$
c_{1}(N)^{2}=-6, c_{1}(M) \cdot \sigma^{2}\left(E_{2}\right)=-1, c_{1}(N) \cdot H=0 \text { and } c_{2}(N)=m-1 .
$$

So Hirzebruch-Riemann-Roch shows that $\chi(N)=\frac{-6}{2}-m+1+3=1-m$.

As $-m>0$ by assumption we must have $\operatorname{dim}\left(H^{0}(Y, N)\right)>0$ or $\operatorname{dim}\left(H^{2}(Y, N)\right)>0$. But we see that

$$
H^{2}(Y, N)=\operatorname{Ext}_{\mathcal{O}_{Y}}^{2}\left(\mathcal{O}_{Y}, N\right)=\operatorname{Ext}_{\mathcal{O}_{Y}}^{2}\left(\mathcal{O}_{Y}\left(\sigma^{2}\left(E_{2}\right)\right), M\right)=\operatorname{Ext}_{A}^{2}\left(A \otimes_{\mathcal{O}_{Y}} \mathcal{O}_{Y}\left(\sigma^{2}\left(E_{2}\right)\right), M\right) .
$$

Using Serre duality and $\omega_{A}=A \otimes_{\mathcal{O}_{Y}} \mathcal{O}_{Y}(-H)$ shows that the last group is isomorphic to $\operatorname{Hom}_{A}\left(M, A \otimes_{\mathcal{O}_{Y}} \mathcal{O}_{Y}\left(\sigma^{2}\left(E_{2}\right)-H\right)\right)$. But as

$$
c_{1}\left(A \otimes_{\mathcal{O}_{Y}} \mathcal{O}_{Y}\left(\sigma^{2}\left(E_{2}\right)-H\right)\right)=c_{1}\left(A \otimes_{\mathcal{O}_{Y}} \mathcal{O}_{Y}\left(\sigma^{2}\left(E_{2}\right)\right)\right)-3 H=c_{1}(A)+H-3 H=c_{1}(A)-2 H
$$

we conclude that $c_{1}\left(A \otimes \mathcal{O}_{Y} \mathcal{O}_{Y}\left(\sigma^{2}\left(E_{2}\right)-H\right)\right)-c_{1}(M)=-3 H$, which is not effective.

So the Hom-group is trivial and thus also $H^{2}(Y, N)=0$.

We must have $\operatorname{dim}\left(H^{0}(Y, N)\right)>0$ implying that there is an embedding $A \otimes_{\mathcal{O}_{Y}} \mathcal{O}_{Y}\left(\sigma^{2}\left(E_{2}\right)\right) \hookrightarrow M$, since

$$
H^{0}(Y, N)=\operatorname{Hom}_{\mathcal{O}_{Y}}\left(\mathcal{O}_{Y}, N\right)=H_{o m} \mathcal{O}_{Y}\left(\mathcal{O}_{Y}\left(\sigma^{2}\left(E_{2}\right)\right), M\right)=H o m_{A}\left(A \otimes_{\mathcal{O}_{Y}} \mathcal{O}_{Y}\left(\sigma^{2}\left(E_{2}\right)\right), M\right) .
$$

As both bundles are locally free and have the same first Chern class, they must be isomorphic. But since $c_{2}\left(A \otimes \mathcal{O}_{Y} \mathcal{O}_{Y}\left(\sigma^{2}\left(E_{2}\right)\right)\right)=0>m=c_{2}(M)$ we see that this is impossible.

We conclude that no such $A$-line bundle can exist. So if we have an $A$-line bundle with $c_{1}=$ $c_{1}(A)+H$ then the minimal second Chern class is given by $c_{2}=0$.

For the case $n=2$ we only have partial results:

\section{Lemma 4.26:}

If $M$ is an A-line bundle with $c_{1}=c_{1}(A)+2 H$, then the second Chern class is bounded from below: $c_{2} \geq 3$. 


\section{Proof:}

The $A$-line bundle $A \otimes_{\mathcal{O}_{Y}} \mathcal{O}_{Y}\left(\sigma\left(E_{2}\right)+\sigma^{2}\left(E_{2}\right)\right)$ has Chern classes $c_{1}=c_{1}(A)+2 H$ and $c_{2}=4$. Assume there is an $A$-line bundle $M$ with $c_{1}=c_{1}(A)+2 H$ and $c_{2}=m \leq 2$. Define $N:=$ $M \otimes_{\mathcal{O}_{Y}} \mathcal{O}_{Y}\left(-\sigma\left(E_{2}\right)-\sigma^{2}\left(E_{2}\right)\right)$. Then we can compute:

$$
c_{1}(N)^{2}=-12, c_{1}(N) \cdot H=0 \text { and } c_{2}(N)=m-6
$$

Hirzebruch-Riemann-Roch shows that $\chi(N)=3-m$, but using the same arguments as in the previous lemma, we see that $H^{2}(Y, N)=0$, so that $\operatorname{dim}\left(H^{0}(Y, N)\right)>0$ for $m \leq 2$. We thus have an embedding $A \otimes_{\mathcal{O}_{Y}} \mathcal{O}_{Y}\left(\sigma\left(E_{2}\right)+\sigma^{2}\left(E_{2}\right)\right) \hookrightarrow M$ which must be an isomorphism, as in the previous lemma. But these bundles have different second Chern classes, so this impossible. Thus if $M$ is an $A$-line bundle with $c_{1}=c_{1}(A)+2 H$, then we must have $c_{2} \geq 3$.

Unfortunately we were not able to find an $A$-line bundle with second Chern class $c_{2}=3$. Possibly no such $A$-line bundle exists, in which case $c_{2}=4$ would be the minimal second Chern class, and the corresponding moduli space is not empty, because $A \otimes_{\mathcal{O}_{Y}} \mathcal{O}_{Y}\left(\sigma\left(E_{2}\right)+\sigma^{2}\left(E_{2}\right)\right)$ defines an element in it.

\subsubsection{Moduli spaces of line bundles with minimal second Chern classes}

\section{Lemma 4.27:}

Let $M$ be an A-line bundle with $c_{1}=c_{1}(A)$ and $c_{2}=-1$, then $M \cong A$. The moduli space Pic $(A)$ of such line bundles is a point.

\section{Proof:}

Let $M$ be such an $A$-line bundle. Then $\chi(M)=\chi(A)=1$.

By (4.23) we have $H^{2}(Y, M)=0$ and so $\operatorname{dim}\left(H^{0}(Y, M)\right) \geq 1$. This gives an embedding $A \hookrightarrow M$ which must be an isomorphism because $c_{1}(M)=c_{1}(A)$.

Finally, we see $\operatorname{dim}\left(\operatorname{Ext}_{A}^{1}(A, A)\right)=\operatorname{dim}\left(H^{1}(Y, A)\right)=0$, as $\operatorname{dim}\left(H^{0}(Y, A)\right)=1$ by (1.50) and $\operatorname{dim}\left(H^{2}(Y, A)\right)=0$ since $A$ defines a terminal del Pezzo order on $\mathbb{P}^{2}$, see (3.5).

Thus the moduli space $\operatorname{Pic}(A)$ is a point.

\section{Lemma 4.28:}

Let $M$ be an A-line bundle with $c_{1}=c_{1}(A)+H$ and $c_{2}=0$, then $M \cong A \otimes \mathcal{O}_{Y} \mathcal{O}_{Y}\left(\sigma^{2}\left(E_{2}\right)\right)$. The moduli space Pic $(A)$ of such line bundles is a point.

\section{Proof:}

Assume $M$ is such an $A$-line bundle. We define $N:=M \otimes_{\mathcal{O}_{Y}} \mathcal{O}_{Y}\left(-\sigma^{2}\left(E_{2}\right)\right)$, like in the proof of (4.25).

We see that $\chi(N)=1$ and $H^{2}(Y, N)=0$. So we have an embedding

$$
A \otimes \mathcal{O}_{Y} \mathcal{O}_{Y}\left(\sigma^{2}\left(E_{2}\right)\right) \hookrightarrow M
$$

which is in fact an isomorphism by comparing first Chern classes.

It remains to determine $\operatorname{Ext}_{A}^{1}(M, M)$.

But as $A \otimes_{\mathcal{O}_{Y}} \mathcal{O}_{Y}\left(\sigma^{2}\left(E_{2}\right)\right)=\mathcal{O}_{Y}\left(\sigma^{2}\left(E_{2}\right)\right) \oplus \mathcal{O}_{Y}\left(E_{1}\right) \oplus \mathcal{O}_{Y}\left(E_{1}-E_{2}+\sigma\left(E_{1}\right)\right)$, we see using (1.46) that this Ext-group is actually isomorphic to:

$$
H^{1}\left(Y, \mathcal{O}_{Y}\right) \oplus H^{1}\left(Y, \mathcal{O}_{Y}\left(E_{1}-\sigma^{2}\left(E_{2}\right)\right)\right) \oplus H^{1}\left(Y, \mathcal{O}_{Y}\left(E_{1}-E_{2}+\sigma\left(E_{1}\right)-\sigma^{2}\left(E_{2}\right)\right)\right) .
$$


But certainly $H^{1}\left(Y, \mathcal{O}_{Y}\right)=0$ and the other two summands vanish as well.

We will prove this for $K=\mathcal{O}_{Y}\left(E_{1}-\sigma^{2}\left(E_{2}\right)\right)$. Hirzebruch-Riemann-Roch shows that $\chi(K)=0$. But $H^{0}(Y, K)=0$, because if $K$ had a global section, then $K \cong \mathcal{O}_{Y}(D)$ for some nontrivial effective divisor $D$ linear equivalent to $E_{1}-\sigma^{2}\left(E_{2}\right)$.

Because $H$ is ample we must have $D . H>0$. But since the product only depends on the linear equivalence class of a divisor, we see $D . H=\left(E_{1}-\sigma^{2}\left(E_{2}\right)\right) . H=0$. So such a divisor $D$ does not exist and we have $H^{0}(Y, K)=0$.

A similar argument using Serre duality shows $H^{2}(Y, K)=0$, we conclude that we must have $H^{1}(Y, K)=0$.

So $\operatorname{Ext}_{A}^{1}(M, M)=0$ and the moduli space $\operatorname{Pic}(A)$ is a point. 


\section{Moduli spaces on the projective plane: Calabi-Yau orders}

\subsection{Construction of an order and some cohomological computations}

In this chapter we want to study moduli spaces of $\mathcal{A}$-line bundles, where $\mathcal{A}$ is a terminal CalabiYau order on $\mathbb{P}^{2}$. We will use the results found in [BA12] to find a Calabi-Yau order $\mathcal{A}$ on $\mathbb{P}^{2}$ of rank four ramified in a smooth sextic $D$. This order arises as a noncommutative cyclic cover. To use the noncommutative cyclic covering trick we need to find a double cover $\pi: Y \rightarrow \mathbb{P}^{2}$ ramified on $D$. This time we start with $Y$, find a group of automorphisms $G \subset A u t(Y)$ such that $Y / G=\mathbb{P}^{2}$ and so that the map $\pi: Y \rightarrow Y / G$ ramifies over a smooth sextic $D$. This is possible, because almost everything we look for is determined by the Picard lattice of the $K 3$ surface.

It is known that for any smooth $K 3$ surface $X$ we have $\Lambda:=H^{2}(X, \mathbb{Z})=\mathbb{Z}^{22}$. If we equip this lattice with the cup product, then it is called the $K 3$ lattice and it is isomorphic to:

$$
\Lambda=\mathbb{E} \perp \mathbb{E} \perp \mathbb{H} \perp \mathbb{H} \perp \mathbb{H},
$$

here $\mathbb{E}$ is the negative of the usual $\mathbb{E}_{8}$-lattice and $\mathbb{H}$ is a hyperbolic plane. Using the exponential sequence:

$$
0 \longrightarrow \mathbb{Z} \longrightarrow \mathcal{O}_{Y} \longrightarrow \mathcal{O}_{Y}^{\times} \longrightarrow 0
$$

and the fact that $H^{1}\left(Y, \mathcal{O}_{Y}\right)=0$ show that we have an embedding $\operatorname{Pic}(Y) \hookrightarrow \Lambda$. Furthermore we have the following theorem due to Morrison:

Theorem 5.1 ([BA12, Proposition 3.4]):

Assume $S \hookrightarrow \Lambda$ is a primitive sublattice, that is $\Lambda / S$ is torsion-free, with rank $\rho$ and signature $(1, \rho-1)$. Then there exists a K3 surface $Y$ and an isometry $\operatorname{Pic}(Y) \cong S$.

We set $S=\mathbb{Z}^{3}=\left\langle S_{1}, S_{2}, S_{3}\right\rangle$ and define the intersection form on $S$ by the matrix

$$
\left(\begin{array}{ccc}
-2 & 3 & 0 \\
3 & -2 & 1 \\
0 & 1 & -2
\end{array}\right) .
$$

In [BA12] it is shown that $S \hookrightarrow \Lambda$ is a primitive sublattice with signature $(1,2)$. So we can find a $K 3$ surface $Y$ with $S$ as its Picard lattice. The next step is to see that the automorphism of $S$ given by:

$$
\left(\begin{array}{ccc}
0 & 1 & 1 \\
1 & 0 & 1 \\
0 & 0 & -1
\end{array}\right)
$$

extends to a so-called effective Hodge isometry between $H^{2}(Y, \mathbb{Z})$ and $H^{2}(Y, \mathbb{Z})$. The next theorem, the Strong Torelli theorem, states that this isometry actually comes from an automorphism of $Y$ :

Theorem 5.2 ([WCdV84, Theorem VIII.11.1]):

Assume $\phi: H^{2}(Y, \mathbb{Z}) \rightarrow H^{2}\left(Y^{\prime}, \mathbb{Z}\right)$ is an effective Hodge isometry between two K3 surfaces $Y$ and $Y^{\prime}$. Then there is a unique biholomorphic morphism $\sigma: Y^{\prime} \rightarrow Y$ such that $\sigma^{*}=\phi$. 
The next result in [BA12] states that if we put $G=\langle\sigma>$ then $\pi: Y \rightarrow Y / G$ is a double cover with $Y / G=\mathbb{P}^{2}$. Furthermore one has $H^{0}(G, \operatorname{Pic}(Y))=\operatorname{Pic}(Y)^{G}=\pi^{*} \operatorname{Pic}(Y / G)$ and $H^{1}(G, \operatorname{Pic}(Y))=\mathbb{Z} / 2 \mathbb{Z}$. The last group is generated by $L=\mathcal{O}_{Y}\left(S_{1}-S_{3}\right)$.

Finaly one computes that $\pi$ ramifies over a smooth sextic $D$ with 2 tritangents $l_{1}$ and $l_{2}$. Especially we have a decomposition $\pi^{-1}\left(l_{1}\right)=S_{1} \cup \sigma\left(S_{1}\right)=S_{1} \cup S_{2}$.

We see that $\phi: L_{\sigma}^{2} \stackrel{\sim}{\longrightarrow} \mathcal{O}_{Y}$ and that $\phi$ satisfies the overlap condition. So

$$
A:=\mathcal{O}_{Y} \oplus L_{\sigma}
$$

is a cyclic algebra which defines a Calabi-Yau order $\mathcal{A}$ on $\mathbb{P}^{2}$ ramified on $D$. This shows that $\mathcal{A}$ is in fact a terminal Calabi-Yau order. As noted in (4.1) we will work completely on $Y$ so that from now on we will only use $A$.

Since $\pi$ is a double cover ramified over a smooth sextic, we see that the ramification divisor in $Y$ is given by $3 H$ where $H:=\pi^{*} l$ for a general line $l \subset \mathbb{P}^{2}$. We remark that $H$ is an ample divisor on $Y$.

Using the same method as in [CK11, Proposition 4.1], we see that the $\mathcal{O}_{Y}$-module associated to $\omega_{A}$ is given by:

$$
\omega_{\mathbb{P}^{2}} \otimes_{\mathcal{O}^{2}}\left(A \otimes_{\mathcal{O}_{Y}} \mathcal{O}_{Y}(R)\right) \cong \mathcal{O}_{Y}(-3 H) \otimes_{\mathcal{O}_{Y}}\left(A \otimes_{\mathcal{O}_{Y}} \mathcal{O}_{Y}(R)\right) \cong A \otimes_{\mathcal{O}_{Y}} \mathcal{O}_{Y}(-3 H+R) \cong A
$$

The first isomorphism uses the projection formula for $\pi_{*}$, see [Har77, Exercise II.5.1 (d)], and $\omega_{\mathbb{P}^{2}}=\mathcal{O}_{\mathbb{P}^{2}}(-3 l)$. The second isomorphism uses the fact that $H$ is $G$-invariant, thus implying $\mathcal{O}_{Y}(H) \otimes_{\mathcal{O}_{Y}} A \cong A \otimes_{\mathcal{O}_{Y}} \mathcal{O}_{Y}(H)$. We recall that the Hirzebruch-Riemann-Roch formula for a rank $r$ vector bundle $M$ with Chern classes $c_{1}$ and $c_{2}$ on a $K 3$-surface is given by:

$$
\chi(M)=\frac{c_{1}^{2}}{2}-c_{2}+2 r .
$$

First we will compute some cohomology groups of line bundles on $Y$. These results will be needed later.

\section{Lemma 5.3:}

The line bundle $L=\mathcal{O}_{Y}\left(S_{1}-S_{3}\right)$ has vanishing cohomology, that is $H^{i}(Y, L)=0$ for $i=0,1,2$.

\section{Proof:}

Assume the contrary, that is $\operatorname{dim}\left(H^{0}(Y, L)\right)>0$. Then there is a nontrivial effective divisor $D$ on $Y$, linearly equivalent to $S_{1}-S_{3}$, such that $L \cong \mathcal{O}_{Y}(D)$.

Now $H$ is ample and $\left(S_{1}-S_{3}\right) \cdot H=0$ so we see that $D . H=0$ since the intersection product only depends on the linear equivalence class of $D$.

But as $D$ is nontrivial and effective and $H$ is ample, we must have D.H $>0$, see [Fri98].

We conclude that $H^{0}(Y, L)=0$ and equivalently we see that $H^{2}(Y, L)=H^{0}\left(Y, L^{-1}\right)=0$ using Serre duality.

Since $c_{1}(L)=S_{1}-S_{3}$ and $c_{2}(L)=0$ we get

$$
c_{1}^{2}=S_{1}^{2}+2 S_{1} S_{3}+S_{3}^{2}=-2+0-2=-4 .
$$

So Hirzebruch-Riemann-Roch gives $\chi(L)=\frac{-4}{2}-0+2=0$.

Using $H^{0}(Y, L)=H^{2}(Y, L)=0$ shows that we also must have $H^{1}(Y, L)=0$.

\section{Lemma 5.4:}

The line bundle $N=\mathcal{O}_{Y}\left(S_{2}-S_{3}\right)$ has cohomology $H^{i}(Y, N)=\left\{\begin{array}{ll}k, & \text { if } i=1 \\ 0, & \text { if } i=0,2\end{array}\right.$. 


\section{Proof:}

As in the previous lemma we see that $H^{0}(Y, N)=H^{2}(Y, N)=0$ since $\left(S_{2}-S_{3}\right) \cdot H=0$.

We have $c_{1}(N)=S_{2}-S_{3}$ and $c_{2}(N)=0$ implying:

$$
c_{1}^{2}=S_{2}^{2}-2 S_{2} S_{3}+S_{3}^{2}=-2-2-2=-6 .
$$

Hirzebruch-Riemann-Roch gives us $\chi(N)=-1$.

Using $H^{0}(Y, N)=H^{2}(Y, N)=0$ shows that we must have $H^{1}(Y, N)=k$.

\subsection{Possible Chern classes}

In this section we want to find the possible Chern classes of a $A$-line bundle $M$ as an $\mathcal{O}_{Y}$-module. To do this we will use the following proposition:

Proposition 5.5 ([CK11, Proposition 3.6]):

Assume $M$ is an $A$-line bundle, where $A$ is a cyclic algebra of the form $A=\mathcal{O}_{Y} \oplus L_{\sigma}$ where $\phi: L_{\sigma}^{2} \stackrel{\sim}{\longrightarrow} \mathcal{O}_{Y}$ is an isomorphism. Then $M$ is $H$-semistable as an $\mathcal{O}_{Y}$-module, where $H$ is an ample G-invariant divisor on $Y$.

Since $M$ is an $A$-line bundle we extract from the proof of [CK11, Theorem 3.3] that the multiplication morphism $L_{\sigma} \otimes_{\mathcal{O}_{Y}} M \rightarrow M$ is an isomorphism.

Using these two results we can prove the following lemma.

\section{Lemma 5.6:}

If $M$ is an A-line bundle, then $c_{1}(M)=L+n H$ for some $n \in \mathbb{Z}$.

\section{Proof:}

By definition we have $L_{\sigma} \otimes_{\mathcal{O}_{Y}} M=L \otimes_{\mathcal{O}_{Y}} \sigma^{*} M$.

So the second fact above shows that we have an isomorphism of $\mathcal{O}_{Y \text {-modules: }}$

$$
L \otimes \mathcal{O}_{Y} \sigma^{*} M \cong M .
$$

Applying $c_{1}$ and using the properties of $c_{1}$ shows:

$$
2 c_{1}(L)+\sigma^{*} c_{1}(M)=c_{1}(M) .
$$

Equivalently this can be written as:

$$
c_{1}(M)-\sigma^{*} c_{1}(M)=2 c_{1}(L) .
$$

If we have two solutions for $c_{1}(M)$, then the last equation shows that their difference is an element of $\operatorname{ker}\left(1-\sigma^{*}\right)=\operatorname{Pic}(Y)^{G}$. As we have seen, we have $\operatorname{Pic}(Y)^{G}=\pi^{*} \operatorname{Pic}\left(\mathbb{P}^{2}\right)=\mathbb{Z} H$.

Furthermore we know that $L \otimes_{\mathcal{O}_{Y}} \sigma^{*} L \cong \mathcal{O}_{Y}$, that is $\sigma^{*} L=-L$. This implies that $c_{1}(M)=L$ is a solution to the equation (18).

So if $M$ is an $A$-line bundle, then as an $\mathcal{O}_{Y}$-module there is an $n \in \mathbb{Z}$ such that:

$$
c_{1}(M)=L+n H .
$$

Since $c_{1}\left(M \otimes \mathcal{O}_{Y}(H)\right)=c_{1}(M)+2 H$, it is enough to consider the cases $n=0$ and $n=1$ in the previous lemma, see [Ler11, Remark 3.8]. Since by (5.5) any $A$-line bundle is a $H$-semistable vector bundles of rank 2 on $Y$, we can use Bogomolov's inequality on $Y$ to see that we have: 


$$
\Delta(M)=4 c_{2}(M)-c_{1}(M)^{2} \geq 0
$$

This bounds the second Chern class for an $A$-line bundle from below. We are now interested in finding the minimal second Chern classes.

\section{Lemma 5.7:}

If $M$ is an A-line bundle with $c_{1}(M)=L$, then the minimal second Chern class is $c_{2}=0$.

\section{Proof:}

We have $c_{1}(M)=L$, implying $c_{1}^{2}=-4$.

Bogomolov's inequality shows that $\Delta(M)=4 c_{2}+4 \geq 0$, which implies:

$$
c_{2}(M) \geq-1 \text {. }
$$

But this is not the best lower bound, which we will now see.

Assume there is an $A$-line bundle with $c_{1}(M)=L$ and $c_{2}(M)=-1$, then Hirzebruch-RiemannRoch shows:

$$
\chi(M)=\frac{L^{2}}{2}+1+4=-2+1+4=3 .
$$

So we must have $\operatorname{dim}\left(H^{0}(Y, M)\right)>0$ or $\operatorname{dim}\left(H^{2}(Y, M)\right)>0$.

If $\operatorname{dim}\left(H^{0}(Y, M)\right)>0$ we have an embedding $A \hookrightarrow M$. To see this, we note that because $M$ is an $A$-module, by (1.46), we have:

$$
H^{0}(Y, M)=\operatorname{Hom}_{\mathcal{O}_{Y}}\left(\mathcal{O}_{Y}, M\right)=\operatorname{Hom}_{A}(A, M) .
$$

So we get a nontrivial morphism $A \rightarrow M$, which must be injective by (1.47). As $A$ and $M$ are both locally free and $c_{1}(A)=c_{1}(M)$, we see that they must already be isomorphic.

However $c_{2}(A) \neq c_{2}(M)$, which is impossible if they are isomorphic.

So we must have $H^{0}(Y, M)=0$, implying $\operatorname{dim}\left(H^{2}(Y, M)>0\right.$.

Since $M$ is an $A$-module, the last group is by (1.46) just

$$
H^{2}(Y, M)=\operatorname{Ext}_{\mathcal{O}_{Y}}^{2}\left(\mathcal{O}_{Y}, M\right)=\operatorname{Ext}_{A}^{2}(A, M) .
$$

Using Serre duality and the fact that $\omega_{A}=A$ shows that:

$$
H^{2}(Y, M)=\operatorname{Ext}_{A}^{2}(A, M)=\operatorname{Hom}_{A}\left(M, \omega_{A}\right)^{\prime}=\operatorname{Hom}_{A}(M, A)^{\prime} .
$$

So as $H^{2}(Y, M) \neq 0$, we get an embedding $M \hookrightarrow A$.

But again $c_{1}(M)=c_{1}(A)$ so these modules must be isomorphic, which is impossible since they have diffrent second Chern classes. We conclude $H^{2}(Y, M)=0$.

But $H^{0}(Y, M)=H^{2}(Y, M)=0$ cannot happen, as $\chi(M)>0$. So no such $A$-module can exist. That is if $M$ is an $A$-module with $c_{1}(M)=L$ then:

$$
c_{2}(M) \geq 0
$$

\section{Lemma 5.8:}

If $M$ is an A-line bundle with $c_{1}(M)=L+H$, then the minimal second Chern class is $c_{2}=1$.

\section{Proof:}

As $c_{1}(M)=L+H$, we compute 


$$
c_{1}^{2}=L^{2}+2 L H+H^{2}=-4+0+2=-2 .
$$

By Bogomolov we have $\Delta(M)=4 c_{2}+2 \geq 0$ and since $c_{2} \in \mathbb{Z}$ we see that

$$
c_{2}(M) \geq 0 \text {. }
$$

Again this is not the best lower bound.

Assume there is an $A$-line bundle $M$ with $c_{1}(M)=L+H$ and $c_{2}(M)=0$.

Define $N:=M \otimes_{\mathcal{O}_{Y}} \mathcal{O}_{Y}\left(-S_{1}\right)$. Then we get by (1.79):

$$
c_{1}(N)=c_{1}(M)-2 S_{1} \text { and } c_{2}(N)=c_{2}(M)-c_{1}(M) S_{1}+S_{1}^{2} .
$$

Computing everything gives:

$$
c_{1}(N)=-S_{1}-S_{3}+H \text { and } c_{2}(N)=-1 .
$$

Because $c_{1}(N)^{2}=-6$ we get $\chi(N)=2$ by Hirzebruch-Riemann-Roch.

This implies that $\operatorname{dim}\left(H^{0}(Y, N)\right)>0$ or $\operatorname{dim}\left(H^{2}(Y, N)\right)>0$.

If $\operatorname{dim}\left(H^{0}(Y, N)\right)>0$, using (1.46), we see that:

$$
H^{0}(Y, N)=\operatorname{Hom}_{\mathcal{O}_{Y}}\left(\mathcal{O}_{Y}, N\right)=H_{\operatorname{Hom}_{\mathcal{O}_{Y}}}\left(\mathcal{O}_{Y}\left(S_{1}\right), M\right)=H o m_{A}\left(A \otimes_{Y} \mathcal{O}_{Y}\left(S_{1}\right), M\right) .
$$

We conclude that there is an embedding $A \otimes_{Y} \mathcal{O}_{Y}\left(S_{1}\right) \hookrightarrow M$ of $A$-line bundles. But

$$
c_{1}\left(A \otimes_{Y} \mathcal{O}_{Y}\left(S_{1}\right)\right)=L+H=c_{1}(M)
$$

and $A \otimes_{Y} \mathcal{O}_{Y}\left(S_{1}\right)$ and $M$ are locally free, so they must be isomorphic. This is not possible, because $c_{2}\left(A \otimes_{Y} \mathcal{O}_{Y}\left(S_{1}\right)\right)=1 \neq 0=c_{2}(M)$.

We conclude that $H^{0}(Y, N)=0$ and thus $\operatorname{dim}\left(H^{2}(Y, N)\right)>0$.

In this case we see that

$$
H^{2}(Y, N)=\operatorname{Ext}_{\mathcal{O}_{Y}}^{2}\left(\mathcal{O}_{Y}, N\right)=\operatorname{Ext}_{\mathcal{O}_{Y}}^{2}\left(\mathcal{O}_{Y}\left(S_{1}\right), M\right)=\operatorname{Ext}_{A}^{2}\left(A \otimes_{Y} \mathcal{O}_{Y}\left(S_{1}\right), M\right) .
$$

Serre duality and $\omega_{A}=A$ imply that $\operatorname{dim}\left(\operatorname{Hom}_{A}\left(M, A \otimes_{Y} \mathcal{O}_{Y}\left(S_{1}\right)\right)\right)>0$. There must be an embedding $M \hookrightarrow A \otimes_{Y} \mathcal{O}_{Y}\left(S_{1}\right)$.

Again this would imply that $M$ and $A \otimes_{Y} \mathcal{O}_{Y}\left(S_{1}\right)$ are isomorphic because they have the same first Chern class. But since their second Chern classes differ, this cannot happen.

Putting everything together we see that there cannot be an $A$-module with the desired Chern classes.

So if $M$ is an $A$-line bundle with $c_{1}=L+H$ then we must have $c_{2}(M) \geq 1$.

\subsection{Moduli spaces of line bundles for minimal second Chern class}

\section{Theorem 5.9:}

Let $M$ be an A-line bundle with $c_{1}=L$ and $c_{2}=0$, then $M \cong A$. The moduli space Pic $(A)$ of such line bundles is a point.

\section{Proof:}

Hirzebruch-Riemann-Roch gives us $\chi(M)=-2+0+4=2>0$. So $\operatorname{dim}\left(H^{0}(Y, M)\right)>0$ or $\operatorname{dim}\left(H^{2}(Y, M)\right)>0$.

If $\operatorname{dim}\left(H^{0}(Y, M)\right)>0$ then we get an embedding $A \hookrightarrow M$ which must be an isomorphism by comparing their first Chern classes. 
If $\operatorname{dim}\left(H^{2}(Y, M)\right)>0$ we get, by using Serre duality $\operatorname{Ext}_{A}^{2}(A, M)=\operatorname{Hom}_{A}(M, A)^{\prime}$, an embed$\operatorname{ding} M \hookrightarrow A$, which is an isomorphism.

In both cases we see that $M \cong A$.

Finally, we have to compute $\operatorname{Ext}_{A}^{1}(A, A)$. But

$$
\operatorname{Ext}_{A}^{1}(A, A)=\operatorname{Ext}_{\mathcal{O}_{Y}}^{1}\left(\mathcal{O}_{Y}, A\right)=H^{1}(Y, A)=H^{1}\left(Y, \mathcal{O}_{Y} \oplus L_{\sigma}\right)=H^{1}\left(Y, \mathcal{O}_{Y}\right) \oplus H^{1}(Y, L),
$$

which by (5.3) shows that $\operatorname{Ext}_{A}^{1}(A, A)=0$.

We see that as in all previous examples, the moduli space of $A$-line bundles containing $A$ itself is just a reduced point.

The situation in the second cases seems not that simple:

\section{Theorem 5.10:}

Let $M$ be an A-line bundle with $c_{1}=L+H$ and $c_{2}=1$, then $M \cong A \otimes \mathcal{O}_{Y} \mathcal{O}_{Y}\left(S_{1}\right)$.

\section{Proof:}

We look at the vector bundle $N:=M \otimes_{Y} \mathcal{O}_{Y}\left(-S_{1}\right)$, like in (5.8) and we see that:

$$
c_{1}(N)=-\left(S_{1}+S_{3}\right)+H \text { and } c_{2}(N)=0 .
$$

Hirzebruch-Riemann-Roch gives us $\chi(N)=-3+0+4=1>0$.

So we have $\operatorname{dim}\left(H^{0}(Y, N)\right)>0$ or $\operatorname{dim}\left(H^{2}(Y, N)\right)>0$.

If $\operatorname{dim}\left(H^{0}(Y, N)\right)>0$, we have:

$$
H^{0}(Y, N)=H_{o m} \mathcal{O}_{Y}\left(\mathcal{O}_{Y}, N\right)=H_{\mathcal{O}_{Y}}\left(\mathcal{O}_{Y}\left(S_{1}\right), M\right)=H o m_{A}\left(A \otimes_{Y} \mathcal{O}_{Y}\left(S_{1}\right), M\right)
$$

This gives an embedding $A \otimes_{Y} \mathcal{O}_{Y}\left(S_{1}\right) \hookrightarrow M$, which must be an isomorphism, by looking at the first Chern classes.

If $H^{0}(Y, N)=0$, then we must have $\operatorname{dim}\left(H^{2}(Y, N)>0\right.$.

In this case we see that we have:

$$
H^{2}(Y, N)=\operatorname{Ext}_{\mathcal{O}_{Y}}^{2}\left(\mathcal{O}_{Y}, N\right)=\operatorname{Ext}_{\mathcal{O}_{Y}}^{2}\left(\mathcal{O}_{Y}\left(S_{1}\right), M\right)=\operatorname{Ext}_{A}^{2}\left(A \otimes_{Y} \mathcal{O}_{Y}\left(S_{1}\right), M\right) .
$$

Using Serre duality $\operatorname{Ext}_{A}^{2}\left(A \otimes_{Y} \mathcal{O}_{Y}\left(S_{1}\right), M\right)=\operatorname{Hom}_{A}\left(M, A \otimes_{Y} \mathcal{O}_{Y}\left(S_{1}\right)\right)^{\prime}$ shows that there is an embedding $M \hookrightarrow A \otimes_{Y} \mathcal{O}_{Y}\left(S_{1}\right)$, which must be an isomorphism.

\section{Lemma 5.11:}

The dimension of the tangent space of the moduli space at $[M]=\left[A \otimes_{\mathcal{O}_{Y}} \mathcal{O}_{Y}\left(S_{1}\right)\right]$ is one.

\section{Proof:}

The Kodaira-Spencer map gives an isomorphism:

$$
T_{[M]} M_{\mathcal{A} / \mathbb{P}^{2} ; c_{1}, c_{2}} \cong \operatorname{Ext}_{\mathcal{A}}^{1}(M, M) .
$$

But, using (1.46), we can compute that:

$$
\operatorname{Ext}_{A}^{1}\left(A \otimes_{Y} \mathcal{O}_{Y}\left(S_{1}\right), A \otimes_{Y} \mathcal{O}_{Y}\left(S_{1}\right)\right)=H^{1}\left(Y, \mathcal{O}_{Y}\right) \oplus H^{1}(Y, N) .
$$

This shows that $\operatorname{Ext}_{A}^{1}(M, M)=k$.

So the topological space of the moduli space consits of a single point. But its scheme structure is harder to find. Since the dimension of the tangent space is one, the moduli space should look like $\operatorname{Spec}\left(k[\epsilon] /\left(\epsilon^{n}\right)\right)$ for some $n \geq 2$. Unfortunately we were not able to determine this $n$ exactly. 


\section{A Azumaya algebras and Brauer groups}

Let $k$ be any field.

\section{Definition A.1:}

A finite dimensional k-algebra $A$ is called Azumaya algebra, if it is a central simple $k$-algebra. Here

- $A$ is a central $k$-algebra, if the center $Z(A)=\{a \in A \mid a b=b a$ for all $b \in A\}$ is $k$;

- $A$ is a simple $k$-algebra, if the only two sided ideals of $A$ are (0) and $A$.

The structure of an Azumaya algebra is well known due to the following theorem of Artin and Wedderburn:

\section{Theorem A.2:}

Assume $A$ is an Azumaya algebra over $k$. Then there is an $n \in \mathbb{N}$ and a finite dimensional division ring $D$ over $k$ such that $A$ is isomorphic to the $n \times n$ matrix ring over $D, A \cong M_{n}(D)$.

\section{Definition A.3:}

Let $A$ and $B$ be Azumaya algebras over $k$, then we say $A$ and $B$ are similar, $A \sim B$, if there are $n$ and $m$ in $\mathbb{N}$ and an isomorphism $A \otimes_{k} M_{n}(k) \cong B \otimes_{k} M_{m}(k)$.

Similarity defines an equivalence relation on the isomorphism classes of Azumaya algebras over $k$. We write $[A]:=\{B \mid B \sim A\}$ for an equivalence class.

\section{Definition A.4:}

Let $[A]$ and $[B]$ be equivalence classes of Azumaya algebras over $k$. Define a multiplication by $[A] \cdot[B]=\left[A \otimes_{k} B\right]$, then this multiplication is well-defined, associative and commutative. Furthermore $[K]$ is the unit element and $[A]^{-1}=\left[A^{\text {op }}\right]$ (here $A^{\text {op }}$ is the opposite ring, that is the same additive group as $A$ but with multiplication perfomed in reverse order). This multiplication defines a group structure on the set of equivalence classes of Azumaya algebras over $k$. This group is called $\operatorname{Br}(k)$, the Brauer group of $k$.

\section{Example A.5:}

The matrix ring $M_{n}(k)$ is an Azumaya algebra over $k$ for any $n \in \mathbb{N}$. For $k=\mathbb{R}$ the Hamilton quaternions $\mathbb{H}$, see (1.40), is an Azumaya algebra over $\mathbb{R}$. This algebra also defines a nontrivial element $[\mathbb{H}]$ in $\operatorname{Br}(\mathbb{R})$. One can in fact show that $\operatorname{Br}(\mathbb{R})=\{[\mathbb{R}],[\mathbb{H}]\} \cong \mathbb{Z} / 2 \mathbb{Z}$. If $k$ is algebraically closed then every finite dimensional division ring over $k$ is trivial, this implies $\operatorname{Br}(k)=\{[k]\}$ by (A.2). One famous result due to Tsen states that if $k$ is algebraically closed and $k(C)$ is the function field of an algebraic curve $C$ over $k$, then $\operatorname{Br}(k(C))=\{[k(C)]\}$.

Now assume $X$ is a noetherian, normal and integral scheme.

\section{Definition A.6:}

Assume $\mathcal{A}$ is a coherent sheaf of $\mathcal{O}_{X}$-algebras. Then $\mathcal{A}$ is called a sheaf of Azumaya algebras if $\mathcal{A}$ is locally free and for every closed point $x \in X \mathcal{A} \otimes_{\mathcal{O}_{X}} k(x)$ is an Azumaya algebra over the residue field $k(x)$. We also call $\mathcal{A}$ an Azumaya algebra on $X$. 


\section{Remark A.7:}

There are several other definitions for Azumaya algebras on schemes equivalent to the one given here. For example: a coherent sheaf of $\mathcal{O}_{X}$-algebras is an Azumaya algebra if there is a covering $\left(U_{i} \rightarrow X\right)$ for the étale topology on $X$ such that for each $i$, there is an $r_{i}$ and an isomorphism $\mathcal{A} \otimes \mathcal{O}_{X} \mathcal{O}_{U_{i}} \cong M_{r_{i}}\left(\mathcal{O}_{U_{i}}\right)$. See [Mil80] for more information.

\section{Example A.8:}

If $M$ is a coherent locally free sheaf on $X$, then $\mathcal{A}=\mathcal{E} n d_{\mathcal{O}_{X}}(M)$ is an Azumaya algebra on $X$.

\section{Lemma A.9:}

Assume $f: X \rightarrow Y$ is a morphism of Noetherian, normal and integral schemes and $\mathcal{A}$ is an Azumaya algebra on $Y$. Then $f^{*} \mathcal{A}$ is an Azumaya algebra on $X$.

\section{Proof:}

We see that $f^{*} \mathcal{A}$ has an obvious structre as an $\mathcal{O}_{X^{-}}$-algebra and it is locally free since $\mathcal{A}$ is locally free. We have to show that $f^{*} \mathcal{A} \otimes_{\mathcal{O}_{X}} k(x)$ is an Azumaya algebra over $k(x)$ for every closed point $x \in X$.

Since the question is local we may assume $X=\operatorname{Spec}(S)$ and $Y=\operatorname{Spec}(R)$. Then $\mathcal{A}$ is given by an $R$-algebra $A$ and we see that $f^{*} \mathcal{A}$ is given by $A \otimes_{R} S$. Now let $x \in X$ be a closed point. Then $k(x)$ is a field extension of $k(y)$ for $y=f(x)$. We conclude:

$$
A \otimes_{R} S \otimes_{S} k(x)=A \otimes_{R} k(x)=A \otimes_{R} k(y) \otimes_{k(y)} k(x)
$$

Here $A \otimes_{R} k(y)$ is an Azumaya algebra over $k(y)$ by assumption.

Since being Azumaya is stable under field extensions, we see that $A \otimes_{R} k(y) \otimes_{k(y)} k(x)$ is an Azumaya algebra over $k(y)$.

As in the case of Azumaya algebras over a field $k$, we can define similar algebras: $\mathcal{A}$ and $\mathcal{B}$ are similar if there are two coherent locally free sheaves $M$ and $N$ on $X$ and an isomorphism $\mathcal{A} \otimes_{\mathcal{O}_{X}} \mathcal{E} n d_{\mathcal{O}_{X}}(M) \cong \mathcal{B} \otimes_{\mathcal{O}_{X}} \mathcal{E} n d_{\mathcal{O}_{X}}(N)$. Then $[\mathcal{A}] \cdot[\mathcal{B}]=\left[\mathcal{A} \otimes_{\mathcal{O}_{X}} \mathcal{B}\right]$ defines a group structure on set of equivalence classes of Azumaya algebras on $X$. This gives the Brauer group $\operatorname{Br}(X)$ of the scheme $X$.

\section{Example A.10:}

The Brauer group of the projective plane over an algebraically closed field is trivial, that is we have $\operatorname{Br}\left(\mathbb{P}^{2}\right)=\left\{\left[\mathcal{O}_{\mathbb{P}^{2}}\right]\right\}$.

\section{B Global dimension}

Let $A$ be a ring with unit and let $M$ be a left $A$-module. Since the category $\operatorname{Mod}(A)$ of left $A$-modules has enough projective objects, there is either an exact sequence, called a projective resolution of $M$ of length $n$ (for some $n \in \mathbb{N}$ ),

$$
0 \longrightarrow P_{n} \longrightarrow \ldots \longrightarrow P_{1} \longrightarrow P_{0} \longrightarrow M \longrightarrow 0
$$

where the left modules $P_{i}$ are projective, or there is no such sequence for any $n$. 


\section{Definition B.1:}

We define the left A-projective dimension of $M$ by

$$
\text { l.pd } d_{A}(M):=\min \{n \in \mathbb{N} \mid \text { there exists a projective resolution of } M \text { of length } n\}
$$

and if no such sequence exists for any $n$ we define l.pd $d_{A}(M):=\infty$.

\section{Definition B.2:}

The left global dimension of $A$ is defined by

$$
\operatorname{l.gldim}(A):=\sup \left\{l \cdot p d_{A}(M) \mid M \in \operatorname{Mod}(A)\right\}
$$

\section{Remark B.3:}

Similiar definitions can be made for right modules and right dimensions. The left and right global dimension may differ. Fortunately Auslander showed in [Aus55], that if $A$ is Noetherian, then $\lg \operatorname{gldim}(A)=r . \operatorname{gldim}(A)$ in this case we just write $\operatorname{gldim}(A)$ and can talk about the global dimension.

\section{Remark B.4:}

- If $p d_{A}(M) \leq n$ then $\operatorname{Ext}_{A}^{n+1}(M, N)=0$ for all $A$-modules $N$, so $\operatorname{Ext}_{A}^{i}(M, N)=0$ for all $i \geq n+1$ and all $A$-modules $N$.

- If $\operatorname{gldim}(A) \leq n$ then $\operatorname{Ext}_{A}^{n+1}(M, N)=0$ for all $A$-modules $M$ and $N$, so $\operatorname{Ext}_{A}^{i}(M, N)=0$ for all $i \geq n+1$ and all $A$-modules $M$ and $N$.

\section{Example B.5:}

- If $A=k\left[x_{1}, \ldots, x_{n}\right]$ for some field $k$, then $\operatorname{gldim}(A)=n$. This is Hilbert's famous syzygy theorem.

- If $A$ is a commutative Noetherian local ring, then $A$ is regular if and only if $\operatorname{gldim}(A)<\infty$. In this case $\operatorname{gldim}(A)=\operatorname{dim}(A)$, where $\operatorname{dim}(A)$ is the Krull dimension of $A$. This is a theorem due to Serre.

Assume $X$ is a smooth projective scheme and $\mathcal{A}$ is a sufficiently nice sheaf of Noetherian rings on $X$. Let $M$ be an $\mathcal{A}$-module, coherent as an $\mathcal{O}_{X}$-module.

\section{Definition B.6:}

We define the $\mathcal{A}$-projective dimension of $M$ by $p d_{\mathcal{A}}(M)=\sup \left\{p d_{\mathcal{A}_{x}}\left(M_{x}\right) \mid x \in X\right\}$.

\section{Definition B.7:}

We define the global dimension of $\mathcal{A}$ by $\operatorname{gldim}(\mathcal{A})=\sup \left\{\operatorname{gldim}\left(\mathcal{A}_{x}\right) \mid x \in X\right\}$

\section{Example B.8:}

- If $\mathcal{A}=\mathcal{O}_{X}$ and $M=k(x)$ is the skyscraper sheaf at some point $x \in X$, then $p d_{\mathcal{O}_{X}}(k(x))=$ $\operatorname{dim}(X)$

- If $\mathcal{A}$ is a terminal order, then $\operatorname{gldim}\left(\mathcal{A}_{\eta}\right)=0$ for the generic point $\eta \in X, \operatorname{gldim}\left(\mathcal{A}_{\xi}\right)=1$ for points of codimension one and $\operatorname{gldim}\left(\mathcal{A}_{x}\right)=2$ for closed points $x \in X$. So $\operatorname{gldim}(\mathcal{A})=2$. 


\section{Grothendieck spectral sequence}

Assume $\mathfrak{A}$ and $\mathfrak{B}$ are abelian categories. Let $F: \mathfrak{A} \rightarrow \mathfrak{B}$ be an additive left exact covariant functor.

\section{Definition C.1:}

An object $I \in O b(\mathfrak{A})$ is called injective if $\operatorname{Hom}_{\mathfrak{A}}(-, I)$ is an exact functor.

\section{Definition C.2:}

An object $A \in O b(\mathfrak{A})$ is called $F$-acyclic if $R^{n} F(A)=0$ for all $n \geq 1$, where $R^{n} F$ is the $n$-th right derived functor of $F$.

\section{Theorem C.3:}

Assume $\mathfrak{A}, \mathfrak{B}$ and $\mathfrak{C}$ are abelian categories and $F: \mathfrak{A} \rightarrow \mathfrak{B}, G: \mathfrak{B} \rightarrow \mathfrak{C}$ are additive covariant left exact functors. If $\mathfrak{A}$ and $\mathfrak{B}$ have enough injectives and $F$ maps injective objects to $G$-acyclic objects, then for every object $A$ in $\mathfrak{A}$ there is a spectral sequence:

$$
E_{2}^{p, q}=R^{p} G\left(R^{q} F(A)\right) \Rightarrow R^{p+q}(G F(A)) .
$$

\section{C.1 Local-to-global spectral sequence}

Assume $\mathcal{A}$ is a maximal order on a smooth projective surface $X$.

\section{Lemma C.4:}

An $\mathcal{A}$-module $I$ is injective if and only if for every injection $\iota: M \hookrightarrow N$ of $\mathcal{A}$-modules and any map $f: M \rightarrow I$ there is a map $g: N \rightarrow I$ such that $f=g \circ \iota$.

\section{Lemma C.5:}

Assume $\mathcal{A}$ is an order on a smooth projective surface $X$ and $I$ is an injective $\mathcal{A}$-module. Then $I_{\mid U}$ is an injective $\mathcal{A}_{\mid U^{-}}$module on $U$ for every open subset $U \subset X$.

\section{Proof:}

Given an injection $\iota: M \hookrightarrow N$ of $\mathcal{A}_{\mid U}$-modules on $U$ and a morphism $f: M \rightarrow I_{\mid U}$. We need to find a map $g: N \rightarrow I_{\mid U}$ such that $f=g \circ \iota$.

Since $U \subset X$ is open, we can use the exact functor $j$ ! (extension by zero).

This functor gives an injection $j_{!}(\iota): j_{!}(M) \hookrightarrow j_{!}(N)$ and a map $j_{!}(f): j_{!}(M) \rightarrow j_{!}\left(I_{\mid U}\right)$ of $\mathcal{A}$-modules on $X$.

Now there is a canonical injection $j_{!}\left(I_{\mid U}\right) \hookrightarrow I$ on $X$, see [Har77, Excercise II.1.19 (c)]. So by composing this with $j_{!}(f)$ we get in fact a map $j_{!}(M) \rightarrow I$ of $\mathcal{A}$-modules.

As $I$ is an injective $\mathcal{A}$-module by assumption there is a map $h: j_{!}(N) \rightarrow I$ with $j_{!}(f)=h \circ j_{!}(\iota)$ by (C.4).

Now we use the exact functor $(-)_{\mid U}$ (restriction to $\mathrm{U}$ ).

This defines a map $h_{\mid U}:\left(j_{!}(N)\right)_{\mid U} \rightarrow I_{\mid U}$. But since $j_{\text {! }}$ and $(-)_{\mid U}$ are a pair of adjoint functors we see that we have natural isomorphisms:

$$
\left(j_{!}(N)\right)_{\mid U}=N,\left(j_{!}(f)\right)_{\mid U}=f, \text { and }\left(j_{!}(\iota)\right)_{\mid U}=\iota .
$$

We conclude that $h_{\mid U}: N \rightarrow I_{\mid U}$ satifies $f=h_{\mid U} \circ \iota$, so that $g:=h_{\mid U}$ is the desired map. 


\section{Lemma C.6:}

Assume $\mathcal{A}$ is a maximal order on a smooth projective surface $X$ and $I$ is an injective $\mathcal{A}$-module. Then $\mathcal{H o m}_{\mathcal{A}}(-, I)$ is an exact functor.

\section{Proof:}

Since $\mathcal{H o m}_{\mathcal{A}}(-, I)$ is a left exact contravariant functor, it is enough to show that if $M \hookrightarrow N$ is an injection, then $\mathcal{H o m}_{\mathcal{A}}(N, I) \rightarrow \mathcal{H o m}_{\mathcal{A}}(M, I)$ is surjective. To do that, it is enough to show that for every open subset $U \subset X$ the map $\operatorname{Hom}_{\mathcal{A}_{\mid U}}\left(N_{\mid U}, I_{\mid U}\right) \rightarrow \operatorname{Hom}_{\mathcal{A}_{\mid U}}\left(M_{\mid U}, I_{\mid U}\right)$ is surjective. But this follows from the previous lemma (C.5).

\section{Lemma C.7:}

Assume $\mathcal{A}$ is a maximal order on a smooth projective surface. If $M$ and I are $\mathcal{A}$-modules such that $M$ is flat as an $\mathcal{O}_{X}$-module and $I$ is injective as an $\mathcal{A}$-module, then $\mathcal{H}_{\mathcal{A}}(M, I)$ is an injective $\mathcal{O}_{X}$-module.

\section{Proof:}

We have to show that $\operatorname{Hom}_{\mathcal{O}_{X}}\left(-, \mathcal{H o m}_{\mathcal{A}}(M, I)\right)$ is an exact functor. But we know from the tensor-hom-adjunction that

$$
\operatorname{Hom}_{\mathcal{O}_{X}}\left(-, \mathcal{H o m}_{\mathcal{A}}(M, I)\right)=\operatorname{Hom}_{\mathcal{A}}\left(-\otimes_{\mathcal{O}_{X}} M, I\right)
$$

which is exact, because $\otimes_{\mathcal{O}_{X}} M$ and $\operatorname{Hom}_{\mathcal{A}}(-, I)$ are exact.

\section{Lemma C.8:}

Assume $\mathcal{A}$ is a maximal order on a smooth projective surface $X$ and $M$ is an $\mathcal{A}$-module. Then there is an exact sequence of $\mathcal{A}$-modules:

$$
0 \longrightarrow \mathrm{K} \longrightarrow \mathrm{F} \longrightarrow \mathrm{M} \longrightarrow 0,
$$

where $F$ is flat as an $\mathcal{O}_{X}$-module.

\section{Proof:}

Since $M$ is also an $\mathcal{O}_{X}$-module and every $\mathcal{O}_{X}$-module is a quotient of a flat $\mathcal{O}_{X}$-module, we can find a surjection:

$$
G \longrightarrow M \longrightarrow 0,
$$

where $G$ is a flat $\mathcal{O}_{X}$-module. Applying $\otimes_{\mathcal{O}_{X}} \mathcal{A}$ and using that there is a surjection

$$
M \otimes_{\mathcal{O}_{X}} \mathcal{A} \longrightarrow M \longrightarrow 0
$$

as $M$ is an $\mathcal{A}$-module, we get a surjection:

$$
G \otimes_{\mathcal{O}_{X}} \mathcal{A} \longrightarrow M \longrightarrow 0 .
$$

Then $F:=G \otimes_{\mathcal{O}_{X}} \mathcal{A}$ is an $\mathcal{A}$-module, which is flat as an $\mathcal{O}_{X}$-module because $G$ and $\mathcal{A}$ are flat as $\mathcal{O}_{X}$-modules. Defining $K:=\operatorname{ker}(F \rightarrow M)$ gives the desired exact sequence.

\section{Lemma C.9:}

Assume $\mathcal{A}$ is a maximal order on a smooth projective surface $X$. If $M$ and I are $\mathcal{A}$-modules, such that $I$ is injective, then $\operatorname{Hom}_{\mathcal{A}}(M, I)$ is acyclic for $\Gamma(X,-)$. 


\section{Proof:}

We have to show that $H^{i}\left(X, \mathcal{H o m}{ }_{\mathcal{A}}(M, I)\right)=0$ for all $i \geq 1$.

By the previous lemma (C.8) there is an exact sequence of $\mathcal{A}$-modules:

$$
0 \longrightarrow \mathrm{K} \longrightarrow \mathrm{F} \longrightarrow \mathrm{M} \longrightarrow 0,
$$

where $F$ is a flat $\mathcal{O}_{X}$-module. Since $I$ is injective by (C.6) we get an exact sequence:

$$
0 \longrightarrow \mathcal{H o m}_{\mathcal{A}}(M, I) \longrightarrow \mathcal{H o m}_{\mathcal{A}}(F, I) \longrightarrow \mathcal{H o m}_{\mathcal{A}}(K, I) \longrightarrow 0 .
$$

Using (C.7) shows that $H^{i}\left(X, \mathcal{H o m}_{\mathcal{A}}(F, I)\right)=0$ for all $i \geq 1$, since injective modules are acyclic for $\Gamma(X,-)$.

Looking at the long exact cohomology sequence shows that we have isomorphisms

$$
H^{i}\left(X, \mathcal{H o m}_{\mathcal{A}}(M, I)\right) \cong H^{i-1}\left(X, \mathcal{H o m}_{\mathcal{A}}(K, I)\right)
$$

for all $i \geq 2$ and an exact sequence:

$$
\begin{aligned}
0 \longrightarrow H^{0}\left(X, \mathcal{H o m}_{\mathcal{A}}(M, I)\right) \longrightarrow H^{0}\left(X, \mathcal{H o m}_{\mathcal{A}}(P, I)\right) \\
\longrightarrow H^{0}\left(\mathcal{H o m}_{\mathcal{A}}(K, I)\right) \longrightarrow 0
\end{aligned}
$$

But $H^{0}\left(X, \mathcal{H o m}_{\mathcal{A}}(-, I)\right)=\operatorname{Hom}_{\mathcal{A}}(-, I)$ and since $I$ is injective we know that $\operatorname{Hom}_{\mathcal{A}}(-, I)$ is exact, which shows that $H^{0}\left(X, \mathcal{H o m}_{\mathcal{A}}(F, I)\right) \rightarrow H^{0}\left(\mathcal{H o m}_{\mathcal{A}}(K, I)\right)$ is surjective.

This implies $H^{1}\left(X, \mathcal{H o m}{ }_{\mathcal{A}}(M, I)\right)=0$. Using this result for $K$, the isomorphisms (19) and descending induction shows that we have in fact $H^{i}\left(X, \mathcal{H o m}_{\mathcal{A}}(M, I)\right)=0$ for all $i \geq 1$.

\section{Theorem C.10:}

Assume $\mathcal{A}$ is a maximal order on a smooth projective surface $X$ and let $M$ and $N$ be $\mathcal{A}$-modules. Then there is a local-to-global spectral sequence

$$
E_{2}^{p, q}=H^{p}\left(X, \mathcal{E} x t_{\mathcal{A}}^{q}(M, N)\right) \Rightarrow \operatorname{Ext}_{\mathcal{A}}^{p+q}(M, N) .
$$

\section{Proof:}

We set $\mathfrak{A}=\operatorname{Mod}(\mathcal{A}), \mathfrak{B}=\operatorname{Mod}\left(\mathcal{O}_{X}\right)$ and $\mathfrak{C}=A b$ in (C.3). Since $\mathfrak{A}$ and $\mathfrak{B}$ are categories of modules they have enough injectives.

Lemma (C.9) shows that $F=\mathcal{H o m}_{\mathcal{A}}(M,-)$ maps injective $\mathcal{A}$-modules to acyclic $G=\Gamma(X,-)$ modules.

By definition we have

$$
R^{p} G=H^{p}(X,-), R^{q} F=\mathcal{E} x t_{\mathcal{A}}^{q}(M,-) \text { and } R^{p+q} G F=\operatorname{Ext}_{\mathcal{A}}^{p+q}(M,-) .
$$

So (C.3) gives the desired spectral sequence. 


\section{References}

[AG60] Maurice Auslander and Oscar Goldman. Maximal orders. Transactions of the American Mathematical Society, 97:1-24, 1960.

[Art86] Michael Artin. Maximal orders of global dimension and Krull dimension two. Inventiones Mathematicae, 84(1):195-222, 1986.

[Art91] Igor Artamkin. Deforming torsion-free sheaves on an algebraic surface. Mathematics of the USSR-Izvestiya, 36(3):449-486, 1991.

[Ati56] Michael Atiyah. On the Krull-Schmidt theorem with applications to sheaves. Bulletin de la Société Mathématique de France, 84:307-317, 1956.

[Aus55] Maurice Auslander. On the dimension of modules and algebras (III) - Global dimension. Nagoya Mathematical Journal, 9:67-77, 1955.

[Aus78] Maurice Auslander. Functors and morphisms determined by objects. In Representation theory of algebras, pages 1-244. Lecture Notes in Pure and Applied Mathematics, Vol. 37. Marcel Dekker, 1978.

[BA12] Hugo Bowne-Anderson. The construction of numerically Calabi-Yau orders on projective surfaces. Journal of Algebra, 355(1):128-153, 2012.

[BL04] Christina Birkenhake and Herbert Lange. Complex Abelian Varieties. SpringerVerlag, Second, augmented edition, 2004.

[CC11] Daniel Chan and Kenneth Chan. Rational curves and ruled orders on surfaces. arXiv:1106.6086v1, 2011.

[Cha05] Daniel Chan. Noncommutative cyclic covers and maximal orders on surfaces. Advances in Mathematics, 198:654-683, 2005.

[CI05] Daniel Chan and Colin Ingalls. The minimal model program for orders over surfaces. Inventiones mathematicae, 161:427-452, 2005.

[CK03] Daniel Chan and Rajesh Kulkarni. Del Pezzo orders on surfaces. Advances in Mathematics, 173:144-177, 2003.

[CK05] Daniel Chan and Rajesh Kulkarni. Numerically Calabi-Yau orders on surfaces. Journal of the London Mathematical Society, 72:571-584, 2005.

[CK11] Daniel Chan and Rajesh Kulkarni. Moduli of bundles on exotic del Pezzo orders. American Journal of Mathematics, 133(1):273-293, 2011.

[CT02] Jean-Louis Colliot-Thélène. Exposant et indice d'algèbres simples centrales non ramifiées (avec un appendice par Ofer Gabber). L'Enseignement Mathématique, 48:127$146,2002$.

[DI71] Frank DeMeyer and Edward Ingraham. Separable algebras over commutative rings. Springer-Verlag, 1971. 
[DO88] Igor Dolgachev and David Ortland. Point sets in projective spaces and Theta functions. Société Mathématique de France, 1988.

[EL99] Geir Ellingsrud and Manfred Lehn. Irreducibility of the punctual quotient scheme of a surface. Arkiv för Matematik, 37(2):245-254, 1999.

[Fri98] Robert Friedman. Algebraic surfaces and holomorphic vector bundles. SpringerVerlag, 1998.

[Har77] Robin Hartshorne. Algebraic Geometry. Springer-Verlag, 1977.

[Har92] Joe Harris. Algebraic Geometry - A First course. Springer-Verlag, 1992.

[HL10] Daniel Huybrechts and Manfred Lehn. The geometry of the moduli spaces of sheaves. Cambridge University Press, Second edition, 2010.

[HS05] Norbert Hoffmann and Ulrich Stuhler. Moduli schemes of generically simple Azumaya modules. Documenta Mathematica, 10:369-389, 2005.

[Kob87] Shoshichi Kobayashi. Differential geometry of complex vector bundles. Iwanami Shoten, Publishers and Princeton University Press, 1987.

[Lan83] Herbert Lange. Universal families of extensions. Journal of Algebra, 83:101-112, 1983.

[Ler11] Boris Lerner. Line bundles and curves on a del Pezzo order. arXiv:1110.5683v1, 2011.

[Lie07] Max Lieblich. Moduli of twisted sheaves. Duke Mathematical Journal, 138(1):23-118, 2007.

[Liu02] Qing Liu. Algebraic Geometry and Arithmetic Curves. Oxford University Press, 2002.

[MF82] David Mumford and John Fogarty. Geometric invariant theory. Springer-Verlag, Second enlarged edition, 1982.

[Mil80] James Milne. Étale Cohomology. Princeton University Press, 1980.

[Muk87] Shigeru Mukai. On the moduli space of bundles on K3 surfaces, I. In Vector bundles on algebraic varieties, pages 341-413. Tata Institute of Fundamental Reasearch, 1987.

[Mum74] David Mumford. Abelian Varieties. Tata Institute of Fundamental Reasearch, 1974.

[Ram69] Mark Ramras. Maximal orders over regular local rings of dimension two. Transactions of the American Mathematical Society, 142:457-479, 1969.

[Sil86] Joseph Silverman. The Arithmetic of Elliptic Curves. Springer-Verlag, 1986.

[Tan81] Allen Tannenbaum. The Brauer group and unirationality : An example of ArtinMumford. In Groupe de Brauer, pages 103-128. Springer-Verlag, 1981.

[WCdV84] W.Barth, C.Peters, and A.Van de Ven. Compact complex surfaces. Springer-Verlag, 1984 . 
[Wit04] Olivier Wittenberg. Transcendental Brauer-Manin obstruction on a pencil of elliptic curves. In Arithmetic of Higher-dimensional Algberaic Varieties, pages 259-267. Progress in Mathematics, Vol. 226. Springer Science+Business Media, LLC, 2004.

[Yos06] Kota Yoshioka. Moduli spaces of twisted sheaves on a projective variety. In Moduli Spaces and Arithmetic Geometry (Kyoto, 2004), pages 1-30. Mathematical Society of Japan, 2006. 